\title{
Lakshmibai-Seshadri paths of level-zero shape and one-dimensional sums associated to level-zero fundamental representations
}

\author{
Satoshi Naito and Daisuke Sagaki
}

\begin{abstract}
We give an interpretation of the energy function and classically restricted one-dimensional sums associated to tensor products of level-zero fundamental representations of quantum affine algebras in terms of Lakshmibai-Seshadri paths of level-zero shape.
\end{abstract}

\section{Introduction}

A one-dimensional sum (1dsum for short) is a weighted sum over certain one-dimensional configurations, where the weights are given by the 'energy function', and arose from the study of solvable lattice models in statistical mechanics through Baxter's corner transfer matrix method. However, the crystal basis theory of Kashiwara provided an intrinsic definition of a 1dsum, and a conceptual proof of the fact that, in the infinite lattice size limit, a 1dsum tends to the character of a highest weight module over an affine Lie algebra (see [KKMMNN92]). The purpose of this paper is to give an interpretation of the energy function and hence of 'classically restricted' 1dsums associated to tensor products of certain finite-dimensional irreducible modules, called level-zero fundamental representations, over a quantum affine algebra via Lakshmibai-Seshadri paths (LS paths for short) of level-zero shape.

Let $\mathfrak{g}$ be an affine Lie algebra over $\mathbb{Q}$ with Cartan subalgebra $\mathfrak{h}$, and let $\mathfrak{g}_{I_{0}}$ be its finitedimensional simple Lie subalgebra whose Dynkin diagram (with $I_{0}$ the set of vertices) is obtained from that of $\mathfrak{g}$ (with $I$ the set of vertices) by removing a distinguished 0 vertex. Also, let $U_{q}(\mathfrak{g}) \supset U_{q}\left(\mathfrak{g}_{I_{0}}\right)$ be the quantized universal enveloping algebras associated to $\mathfrak{g} \supset \mathfrak{g}_{I_{0}}$, and let $U_{q}^{\prime}(\mathfrak{g})\left(\subset U_{q}(\mathfrak{g})\right)$ be the quantum affine algebra (without derivation) associated to $\mathfrak{g}$.

In [KR90] (see also [Kir85, Kir87, Kir89] and [KKR88]), Kirillov and Reshetikhin conjectured the existence of a family of finite-dimensional representations of the Yangian $Y\left(\mathfrak{g}_{I_{0}}\right)$ associated to $\mathfrak{g}_{I_{0}}$ for which tensor products of these representations admit the decomposition into irreducible $\mathfrak{g}_{I_{0}}$-modules, with each multiplicity given by a specific sum of products of binomial coefficients. Note that under the widely believed correspondence between the finite-dimensional representations of the Yangian $Y\left(\mathfrak{g}_{0}\right)$ and those of the nontwisted quantum affine algebra $U_{q}^{\prime}(\mathfrak{g})$, this conjecture can be translated into the one for the untwisted quantum affine algebras; both of them are called the Kirillov-Reshetikhin (KR for short) conjecture. The KR conjecture has been the subject of a number of papers (see $[\mathrm{KNT} 02, \S 5.7]$ and references therein). Among them, we would like to mention [Nak03], [Her06], and [Her07], in which the KR conjecture (of 'type I' in the sense of [KNT02, $\S 5.7])$ is first proved in nontwisted $A, D, E$ cases, then in all the nontwisted cases, and finally in the general case.

Received 24 March 2006, accepted in final form 27 February 2008, published online 26 September 2008. 2000 Mathematics Subject Classification 17B37 (primary), 81R50, 82B23 (secondary).

Keywords: crystal bases, Lakshmibai-Seshadri paths, level-zero representations, Kirillov-Reshetikhin modules, quantum affine algebras.

This journal is (c) Foundation Compositio Mathematica 2008. 


\section{S. NAITO AND D. SAGAKI}

Inspired by the seminal paper [KR90], it was conjectured in [HKOTY99] and [HKOTT02] that there exists a family of finite-dimensional irreducible $U_{q}^{\prime}(\mathfrak{g})$-modules $W_{s}^{(i)}$ having crystal bases $\mathcal{B}^{i, s}$ indexed by $i \in I_{0}$ and $s \in \mathbb{Z}_{\geqslant 1}$ for which the decomposition into irreducible $U_{q}\left(\mathfrak{g}_{I_{0}}\right)$-modules is prescribed by a special case of the KR conjecture (of 'type II' in the sense of [KNT02, $\S 5.7]$ ); the $U_{q}^{\prime}(\mathfrak{g})$-module $W_{s}^{(i)}$ is called a KR module, and the crystal $\mathcal{B}^{i, s}$ is called a KR crystal. It should be mentioned that (the roots of) the Drinfeld polynomials of the KR module $W_{s}^{(i)}$ for each $i \in I_{0}$ and $s \in \mathbb{Z}_{\geqslant 1}$ are also specified up to a multiplicative constant (see [HKOTY99, Remark 2.2] and the comment preceding [HKOTY99, Conjecture 2.1]).

Furthermore, in [HKOTY99] and [HKOTT02], a 'fermionic formula' $M$ was defined to be a suitable $q$-analogue of the tensor product multiplicity formula in the KR conjecture (of type II), which can be rewritten as a weighted sum over a set of combinatorial objects (called rigged configurations). Also, a classically restricted $1 \mathrm{dsum} X$ was introduced as the generating function in $q$ of $U_{q}\left(\mathfrak{g}_{I_{0}}\right)$-highest weight elements of a tensor product of the (conjectural) crystals $\mathcal{B}^{i, s}, i \in I_{0}$, $s \in \mathbb{Z}_{\geqslant 1}$, weighted by the energy function. The ' $X=M$ conjecture' proposed in these papers asserts the equality between these two kinds of polynomials in $q^{-1}$ with nonnegative integer coefficients.

The $X=M$ conjecture (including the construction of the crystals $\mathcal{B}^{i, s}$ ) has been studied intensively in recent years (see [Oka07, $\S \S 2.2$ and 5.3] and references therein). Among them, we would like to mention [Kas02], in which level-zero fundamental representations $W\left(\varpi_{i}\right), i \in I_{0}$, of $U_{q}^{\prime}(\mathfrak{g})$ are introduced, where the $\varpi_{i}, i \in I_{0}$, are the level-zero fundamental weights for $\mathfrak{g}$. Furthermore, in [Kas02], it is proved that the level-zero fundamental representations $W\left(\varpi_{i}\right), i \in I_{0}$, are finite-dimensional irreducible $U_{q}^{\prime}(\mathfrak{g})$-modules having 'simple' crystal bases $\mathcal{B}\left(W\left(\varpi_{i}\right)\right)$. Also, the Drinfeld polynomials of $W\left(\varpi_{i}\right)$ are computed in $[\mathrm{Nak04}, \S 3.1]$ (at least in the nontwisted case), and they coincide with those of $W_{1}^{(i)}$ specified in [HKOTY99] and [HKOTT02] for all $i \in I_{0}$. Therefore, by [Nak03], [Her06], and [Her07], the decomposition of $W\left(\varpi_{i}\right)$ into irreducible $U_{q}\left(\mathfrak{g}_{I_{0}}\right)$-modules turns out to be the one for $W_{1}^{(i)}$ prescribed by a special case of the KR conjecture (of type I) for every $i \in I_{0}$. Thus, through enough evidence (see also [Cha01], [HN06], [Kas05], [FL06], and [FL07]), it is confirmed that the level-zero fundamental representation $W\left(\varpi_{i}\right)$ is indeed the (conjectural) KR module $W_{s}^{(i)}$ with $s=1$, and hence the crystal basis $\mathcal{B}\left(W\left(\varpi_{i}\right)\right)$ of $W\left(\varpi_{i}\right)$ is the KR crystal $\mathcal{B}^{i, 1}$ for every $i \in I_{0}$.

Here we recall that most of all the known proofs of the $X=M$ conjecture are given by establishing a weight-preserving bijection from the set of $U_{q}\left(\mathfrak{g}_{I_{0}}\right)$-highest weight elements of a tensor product of $\mathrm{KR}$ crystals $\mathcal{B}^{i, s}$ to the set of rigged configurations (see [Sch07] and references therein; [KSS02], [Sch05], [SSh06], [SSt06], [BFKL06], and also [FSS07] to name a few of the recent ones). In these proofs, the $\mathrm{KR}$ crystal $\mathcal{B}^{i, s}$ is constructed as a certain direct sum (prescribed by the KR conjecture) of highest weight crystals for $U_{q}\left(\mathfrak{g}_{I_{0}}\right)$; the action of the Kashiwara operators corresponding to the 0 vertex are defined on it, by an argument that depends on the type of $\mathfrak{g}_{I_{0}}$ and requires a lot of work based on an explicit combinatorial realization of highest weight crystals for $U_{q}\left(\mathfrak{g}_{I_{0}}\right)$. Hence it is highly desirable to have a type-independent combinatorial realization of the KR crystals $\mathcal{B}^{i, s}$, $i \in I_{0}, s \in \mathbb{Z}_{\geqslant 1}$, and the corresponding realization of the energy function on tensor products of them, which would provide a general approach to the $X=M$ conjecture.

Contrastingly, in Littelmann's path model, the root operators are universally defined for all paths, and hence we obtain a path model for KR modules if we choose the right set of paths; the advantage of this approach is that, when it can be used, it enables us to study KR crystals in a way completely independent of the type of $\mathfrak{g}_{I_{0}}$. In fact, in a series of papers [NS03, NS05, NS06], we studied the crystal $\mathbb{B}(\lambda)$ of all LS paths of shape $\lambda$ for a level-zero integral weight $\lambda$ of the form $\lambda=\sum_{i \in I_{0}} \lambda^{(i)} \varpi_{i}$ with $\lambda^{(i)} \in \mathbb{Z}_{\geqslant 0}$; we showed that the associated crystal $\mathbb{B}(\lambda)_{\mathrm{cl}}$ is isomorphic to the tensor product crystal $\bigotimes_{i \in I_{0}} \mathbb{B}\left(\varpi_{i}\right)_{\mathrm{cl}}^{\otimes \lambda^{(i)}}$, and that each $\mathbb{B}\left(\varpi_{i}\right)_{\mathrm{cl}}$ is isomorphic as a crystal to $\mathcal{B}\left(W\left(\varpi_{i}\right)\right)=\mathcal{B}^{i, 1}$ for $i \in I_{0}$. Hence the crystal $\mathbb{B}(\lambda)_{\mathrm{cl}}$ is isomorphic to the tensor product 


\section{LS PATHS OF LEVEL-ZERO SHAPE AND 1DSUMS}

crystal $\bigotimes_{i \in I_{0}}\left(\mathcal{B}^{i, 1}\right)^{\otimes \lambda^{(i)}}$. Here the crystal $\mathbb{B}(\lambda)_{\mathrm{cl}}$ is defined to be the set of all paths of the form $\operatorname{cl}(\pi)=\operatorname{cl} \circ \pi, \pi \in \mathbb{B}(\lambda)$, where the map cl $: \mathfrak{h}_{\mathbb{R}}^{*} \rightarrow \mathfrak{h}_{\mathbb{R}}^{*} / \mathbb{R} \delta$ is the canonical projection, with $\delta \in \mathfrak{h}^{*}$ the null root of $\mathfrak{g}$ and $\mathfrak{h}_{\mathbb{R}}^{*}:=\mathbb{R} \otimes \mathfrak{Q}^{*}$. Thus, we have an explicit combinatorial realization of tensor products of $\mathrm{KR}$ crystals $\mathcal{B}^{i, s}, i \in I_{0}$, with $s=1$ in the framework of Littelmann's path model.

Now, the remaining task is to give an interpretation of the energy function on tensor products of KR crystals $\mathcal{B}^{i, 1}, i \in I_{0}$, in terms of Littelmann's path model. The purpose of this paper is to provide such an interpretation. Let $\lambda$ be a level-zero integral weight as above. We define a function (called the degree function) Deg: $\mathbb{B}(\lambda)_{\mathrm{cl}} \rightarrow \mathbb{Z}_{\leqslant 0}$ as follows (see $\S 3.1$ for details). For $\eta \in \mathbb{B}(\lambda)_{\mathrm{cl}}$, the degree $\operatorname{Deg}(\eta) \in \mathbb{Z}$ is defined to be (the negative of) the coefficient of the null root $\delta$ in a specific expression of the weight $\pi_{\eta}(1) \in \mathfrak{h}^{*}$ of a certain distinguished element $\pi_{\eta} \in \mathbb{B}_{0}(\lambda)$ such that $\operatorname{cl}\left(\pi_{\eta}\right)=\eta$, where $\mathbb{B}_{0}(\lambda)$ denotes the connected component of $\mathbb{B}(\lambda)$ containing the straight line path: $t \mapsto t \lambda, t \in[0,1]$. Take an arbitrary sequence $\mathbf{i}=\left(i_{1}, i_{2}, \ldots, i_{n}\right)$ of elements of $I_{0}$ (with repetitions allowed). Then, as above, the tensor product crystal $\mathcal{B}^{i_{1}, 1} \otimes \mathcal{B}^{i_{2}, 1} \otimes \cdots \otimes \mathcal{B}^{i_{n}, 1} \simeq \mathbb{B}\left(\varpi_{i_{1}}\right) \otimes \mathbb{B}\left(\varpi_{i_{2}}\right) \otimes \cdots \otimes \mathbb{B}\left(\varpi_{i_{n}}\right)=: \mathbb{B}_{\mathbf{i}}$ is isomorphic to the crystal $\mathbb{B}(\lambda)_{\text {cl }}$ associated to the crystal $\mathbb{B}(\lambda)$ of all LS paths of shape $\lambda:=$ $\sum_{k=1}^{n} \varpi_{i_{k}}$. Our main result (Theorem 4.1.1) of this paper states that the energy function on the tensor product $\mathbb{B}_{\mathbf{i}}$ of $\mathrm{KR}$ crystals with $s=1$ can be identified (through the isomorphism above between $\mathbb{B}_{\mathbf{i}}$ and $\left.\mathbb{B}(\lambda)_{\mathrm{cl}}\right)$ with the degree function Deg on $\mathbb{B}(\lambda)_{\mathrm{cl}}$, up to a specific additive constant (see $\S 4.1$ for its explicit definition). Furthermore, we obtain an expression (Corollary 5.1.1) for classically restricted 1dsums in terms of LS paths. In particular, by restricting ourselves to the case of $A_{\ell-1}^{(1)}$, we obtain an expression (Corollary 5.2.4) for Kostka-Foulkes polynomials in terms of LS paths.

This paper is organized as follows. In $\S 2$, we first fix our notation for quantum affine algebras. Then we briefly review some standard facts on LS path crystals with weight lattice $P$ or $P_{\mathrm{cl}}$, and fundamental results on simple $P_{\mathrm{cl}}$-crystals for quantum affine algebras. In $\S 3$, we define our 'degree function' on $P_{\mathrm{cl}}$-crystals of LS paths of level-zero shape, and show some of its basic properties. In $\S 4$, we first state our main result (Theorem 4.1.1) describing the energy function associated to tensor products of level-zero fundamental representations. Then, we give a proof of it after showing a key proposition to our proof. In $\S 5$, we mention the relation to classically restricted $1 \mathrm{dsums}$ and Kostka-Foulkes polynomials.

\section{Preliminaries}

\subsection{Affine Lie algebras and quantum affine algebras}

Let $A=\left(a_{i j}\right)_{i, j \in I}$ be a generalized Cartan matrix of affine type. Throughout this paper, we assume that the elements of the index set $I$ are numbered as in $[$ Kac90, $\S 4.8$, Tables Aff 1 -Aff 3]. Take a special vertex $0 \in I$ as in these tables, and set $I_{0}:=I \backslash\{0\}$. Let $\mathfrak{g}=\mathfrak{g}(A)$ be the affine Lie algebra associated to the Cartan matrix $A=\left(a_{i j}\right)_{i, j \in I}$ of affine type over the field $\mathbb{Q}$ of rational numbers, and let $\mathfrak{h}$ be its Cartan subalgebra. Note that $\mathfrak{h}=\left(\bigoplus_{j \in I} \mathbb{Q} h_{j}\right) \oplus \mathbb{Q} d$, where $\Pi^{\vee}:=\left\{h_{j}\right\}_{j \in I} \subset \mathfrak{h}$ is the set of simple coroots, and $d \in \mathfrak{h}$ is the scaling element. Also, we denote by $\Pi:=\left\{\alpha_{j}\right\}_{j \in I} \subset \mathfrak{h}^{*}:=$ $\operatorname{Hom}_{\mathbb{Q}}(\mathfrak{h}, \mathbb{Q})$ the set of simple roots, and by $\Lambda_{j} \in \mathfrak{h}^{*}, j \in I$, the fundamental weights; note that $\alpha_{j}(d)=\delta_{j, 0}$ and $\Lambda_{j}(d)=0$ for $j \in I$. Let $\delta=\sum_{j \in I} a_{j} \alpha_{j} \in \mathfrak{h}^{*}$ and $c=\sum_{j \in I} a_{j}^{\vee} h_{j} \in \mathfrak{h}$ be the null root and the canonical central element of $\mathfrak{g}$, respectively. Here we should note that $a_{0}=2$ if $\mathfrak{g}$ is of type $A_{2 \ell}^{(2)}$, and $a_{0}=1$ otherwise. We define the Weyl group $W$ of $\mathfrak{g}$ by $W=\left\langle r_{j} \mid j \in I\right\rangle \subset \operatorname{GL}\left(\mathfrak{h}^{*}\right)$, where $r_{j} \in \mathrm{GL}\left(\mathfrak{h}^{*}\right)$ is the simple reflection associated to $\alpha_{j}$ for $j \in I$, and then define the set $\Delta^{\text {re }}$ of real roots by $\Delta^{\text {re }}=W \Pi$. The set of positive real roots is denoted by $\Delta_{+}^{\text {re }} \subset \Delta^{\text {re }}$. Also, let us denote by $(\cdot, \cdot)$ a nondegenerate, symmetric, $(W-)$ invariant bilinear form on $\mathfrak{h}^{*}$ normalized in such a way that $\left(\alpha_{i}, \alpha_{j}\right) \in \mathbb{Z}$ for all $i, j \in I$. 


\section{S. NAITO AND D. SAGAKI}

We take a dual weight lattice $P^{\vee}$ and a weight lattice $P$ as follows:

$$
P^{\vee}=\left(\bigoplus_{j \in I} \mathbb{Z} h_{j}\right) \oplus \mathbb{Z} d \subset \mathfrak{h} \quad \text { and } \quad P=\left(\bigoplus_{j \in I} \mathbb{Z} \Lambda_{j}\right) \oplus \mathbb{Z} a_{0}^{-1} \delta \subset \mathfrak{h}^{*} .
$$

It is clear that $P$ contains all the simple roots $\alpha_{j}, j \in I$, and that $P \cong \operatorname{Hom}_{\mathbb{Z}}\left(P^{\vee}, \mathbb{Z}\right)$. The quintuplet $\left(A, \Pi, \Pi^{\vee}, P, P^{\vee}\right)$ is called a Cartan datum for the generalized Cartan matrix $A=\left(a_{i j}\right)_{i, j \in I}$ of affine type (see [HK02, Definition 2.1.1]).

Let $\mathfrak{g}_{I_{0}}$ be the canonical finite-dimensional Lie subalgebra of $\mathfrak{g}$ generated by $x_{j}, y_{j}, j \in I_{0}$, and $h_{j}, j \in I$, where $x_{j}$ (respectively, $y_{j}$ ) is a nonzero root vector of $\mathfrak{g}$ corresponding to the simple root $\alpha_{j}$ (respectively, $-\alpha_{j}$ ); note that $\mathfrak{h}_{I_{0}}:=\bigoplus_{j \in I} \mathbb{Q} h_{j}$ is the Cartan subalgebra of $\mathfrak{g}_{I_{0}}$. We denote by $\stackrel{\circ}{W}$ the subgroup of $W$ generated by $r_{j}, j \in I_{0}$, which can be thought of as the Weyl group of the Lie subalgebra $\mathfrak{g}_{I_{0}} \subset \mathfrak{g}$. Let $w_{0} \in \stackrel{\circ}{W}$ denote the longest element of ${ }^{W}$.

Definition 2.1.1. An integral weight $\lambda \in P$ is said to be level-zero if $\lambda(c)=0$. In addition, a level-zero integral weight $\lambda \in P$ is said to be $I_{0}$-dominant (respectively, strictly $I_{0}$-dominant) if $\lambda\left(h_{j}\right) \geqslant 0$ (respectively, $\lambda\left(h_{j}\right)>0$ ) for all $j \in I_{0}$.

For each $i \in I_{0}=I \backslash\{0\}$, we define a level-zero fundamental weight $\varpi_{i} \in P$ by

$$
\varpi_{i}=\Lambda_{i}-a_{i}^{\vee} \Lambda_{0}
$$

Note that the $\varpi_{i}$ is actually a level-zero, $I_{0}$-dominant integral weight; in fact, $\varpi(c)=0$ and $\varpi_{i}\left(h_{j}\right)=$ $\delta_{i, j}$ for $j \in I_{0}$. In particular, the restriction of the $\varpi_{i}, i \in I_{0}$, to the Cartan subalgebra $\mathfrak{h}_{I_{0}}$ of $\mathfrak{g}_{I_{0}}$ can be thought of as the fundamental weights for $\mathfrak{g}_{I_{0}} \subset \mathfrak{g}$. We set

$$
P_{+}^{0}:=\sum_{i \in I_{0}} \mathbb{Z}_{\geqslant 0} \varpi_{i}
$$

Recall from $[\operatorname{Kas} 02,(5.6)]$ (see also [NS08, Lemmas 2.5 and 2.6]) that, for each $\lambda \in P_{+}^{0}$, the equality $W \lambda \cap(\lambda+\mathbb{Z} \delta)=\lambda+\mathbb{Z} d_{\lambda} \delta$ holds for some positive integer $d_{\lambda} \in \mathbb{Z}_{>0}$, and set $\stackrel{Q}{+}_{+}:=\sum_{j \in I_{0}} \mathbb{Z}_{\geqslant 0} \alpha_{j}$. The next lemma follows immediately from [NS08, Lemma 2.3.2 and Remark 4.1.1] and the proof of [NS08, Lemma 2.3.3] by noting the linear independence of $\alpha_{j}, j \in I_{0}$, and $\delta$.

Lemma 2.1.2. Let $\lambda \in P_{+}^{0}$. Then, for each $w \in W$, there exist unique $\beta \in \dot{Q}_{+}$and $k \in \mathbb{Z}$ such that $w \lambda=\lambda-\beta+k d_{\lambda} \delta$. Moreover, $\lambda-\beta=w^{\prime} \lambda$ for some $w^{\prime} \in \stackrel{\circ}{W}$.

Let $\lambda \in P$ be an integral weight, and $\mu, \nu \in W \lambda$. Following [Lit95, $\S 4$ ], we write $\mu>\nu$ if there exist a sequence $\mu=\nu_{0}, \nu_{1}, \ldots, \nu_{n}=\nu$ of elements of $W \lambda$ and a sequence $\xi_{1}, \xi_{2}, \ldots, \xi_{n}$ of positive real roots such that $\nu_{k}=r_{\xi_{k}}\left(\nu_{k-1}\right)$ and such that $\nu_{k-1}\left(\xi_{k}^{\vee}\right) \in \mathbb{Z}_{<0}$ for all $1 \leqslant k \leqslant n$, where $\xi_{k}^{\vee} \in \mathfrak{h}$ denotes the dual root of $\xi_{k} \in \Delta_{+}^{\text {re }}$, and $r_{\xi_{k}}$ denotes the associated reflection; we write $\mu \geqslant \nu$ if $\mu>\nu$ or $\mu=\nu$.

Remark 2.1.3. Let $\lambda \in P_{+}^{0}$, and let $\nu, \nu^{\prime} \in W \lambda$ be such that $\nu>\nu^{\prime}$. Write $\nu$ and $\nu^{\prime}$ as $\nu=\lambda-\beta+k d_{\lambda} \delta$ and $\nu^{\prime}=\lambda-\beta^{\prime}+k^{\prime} d_{\lambda} \delta$ for $\beta, \beta^{\prime} \in \stackrel{Q}{Q}_{+}$and $k, k^{\prime} \in \mathbb{Z}$ (see Lemma 2.1.2), respectively. Then we deduce from [NS08, Remark 2.4.3(1)] that either $k<k^{\prime}$ holds, or $k=k^{\prime}$ and $\beta-\beta^{\prime} \in \stackrel{Q}{Q}_{+} \backslash\{0\}$ holds.

Now, let cl $: \mathfrak{h}^{*} \rightarrow \mathfrak{h}^{*} / \mathbb{Q} \delta$ be the canonical projection from $\mathfrak{h}^{*}$ onto $\mathfrak{h}^{*} / \mathbb{Q} \delta$. We define a classical weight lattice $P_{\mathrm{cl}}$ and a classical dual weight lattice $P_{\mathrm{cl}}^{\vee}$ by

$$
P_{\mathrm{cl}}=\operatorname{cl}(P)=\bigoplus_{j \in I} \mathbb{Z} \operatorname{cl}\left(\Lambda_{j}\right) \quad \text { and } \quad P_{\mathrm{cl}}^{\vee}=\bigoplus_{j \in I} \mathbb{Z} h_{j} \subset P^{\vee} .
$$

We see that $P_{\mathrm{cl}} \simeq P /(\mathbb{Q} \delta \cap P)$, and that $P_{\mathrm{cl}}$ can be identified with $\operatorname{Hom}_{\mathbb{Z}}\left(P_{\mathrm{cl}}^{\vee}, \mathbb{Z}\right)$ as a $\mathbb{Z}$-module by $(\operatorname{cl}(\lambda))(h)=\lambda(h)$ for $\lambda \in P$ and $h \in P_{\mathrm{cl}}^{\vee}$. The quintuple $\left(A, \operatorname{cl}(\Pi), \Pi^{\vee}, P_{\mathrm{cl}}, P_{\mathrm{cl}}^{\vee}\right)$ is called a classical 


\section{LS PATHS OF LEVEL-ZERO SHAPE AND 1DSUMS}

Cartan datum (see $[\mathrm{HK} 02, \S 10.1]$ ). Note that there exists a natural action of the Weyl group $W$ on $\mathfrak{h}^{*} / \mathbb{Q} \delta$ induced by the one on $\mathfrak{h}^{*}$, since $W \delta=\delta$. It is obvious that $w \circ \mathrm{cl}=\operatorname{cl} \circ w$ for all $w \in W$. If we set $\mathfrak{h}^{* 0}:=\left\{\lambda \in \mathfrak{h}^{*} \mid \lambda(c)=0\right\}$, then there exists a (positive definite) symmetric bilinear form $(\cdot, \cdot)_{\mathrm{cl}}$ on $\operatorname{cl}\left(\mathfrak{h}^{* 0}\right)=\mathfrak{h}^{* 0} / \mathbb{Q} \delta$ induced by the restriction to $\mathfrak{h}^{* 0}$ of the nondegenerate, symmetric bilinear form $(\cdot, \cdot)$ on $\mathfrak{h}^{*}$, since $\left(\delta, \mathfrak{h}^{* 0}\right)=\{0\}$.

Definition 2.1.4. An integral weight $\mu \in P_{\mathrm{cl}}$ is said to be level-zero if $\mu(c)=0$. In addition, a level-zero integral weight $\mu \in P_{\mathrm{cl}}$ is said to be $I_{0}$-dominant (respectively, strictly $I_{0}$-dominant) if $\lambda\left(h_{j}\right) \geqslant 0$ (respectively, $\lambda\left(h_{j}\right)>0$ ) for all $j \in I_{0}$.

Remark 2.1.5. Let $\lambda \in P_{+}^{0}$. It is easy to verify that $\operatorname{cl}(W \lambda)=\stackrel{\circ}{W} \operatorname{cl}(\lambda)$ (see the proof of [NS08, Lemma 2.3.3]). Also, we see that $\operatorname{cl}(\lambda)$ is the unique level-zero, $I_{0}$-dominant integral weight in $\operatorname{cl}(W \lambda)=\stackrel{\circ}{W} \operatorname{cl}(\lambda)$, and that $\operatorname{cl}\left(w_{0} \lambda\right)=w_{0} \operatorname{cl}(\lambda)$ is the unique element of $\operatorname{cl}(W \lambda)=\stackrel{\circ}{W} \operatorname{cl}(\lambda)$ such that $\left(\operatorname{cl}\left(w_{0} \lambda\right)\right)\left(h_{j}\right)=\left(w_{0} \operatorname{cl}(\lambda)\right)\left(h_{j}\right) \leqslant 0$ for all $j \in I_{0}$.

Let $U_{q}^{\prime}(\mathfrak{g})$ be the quantized universal enveloping algebra of the affine Lie algebra $\mathfrak{g}$ with weight lattice $P_{\mathrm{cl}}$ over the field $\mathbb{Q}(q)$ of rational functions in $q$. We denote by $x_{j}, y_{j}, j \in I$, and $q^{h}, h \in P_{\mathrm{cl}}^{\vee}$, the canonical generators of $U_{q}^{\prime}(\mathfrak{g})$, where $x_{j}$ (respectively, $y_{j}$ ) corresponds to the simple root $\alpha_{j}$ (respectively, $-\alpha_{j}$ ) for $j \in I$.

\subsection{Crystals of LS paths with weight lattice $\boldsymbol{P}$ or $\boldsymbol{P}_{\mathrm{cl}}$}

A path (with weight in $P$ ) is, by definition, a piecewise linear, continuous map $\pi:[0,1] \rightarrow \mathfrak{h}_{\mathbb{R}}^{*}:=$ $\mathbb{R} \otimes_{\mathbb{Q}} \mathfrak{h}^{*}$ such that $\pi(0)=0$ and $\pi(1) \in P \subset \mathbb{R} \otimes_{\mathbb{Z}} P=\mathfrak{h}_{\mathbb{R}}^{*}$. We denote by $\mathbb{P}$ the set of all paths $\pi:[0,1] \rightarrow \mathfrak{h}_{\mathbb{R}}^{*}$. For each $\pi_{1}, \pi_{2} \in \mathbb{P}$, we define a path $\pi_{1} \pm \pi_{2} \in \mathbb{P}$ by $\left(\pi_{1} \pm \pi_{2}\right)(t)=\pi_{1}(t) \pm \pi_{2}(t)$ for $t \in[0,1]$. For an integral weight $\nu \in P$, let $\pi_{\nu}$ denote the straight line path connecting $0 \in P$ with $\nu \in P$, i.e. $\pi_{\nu}(t):=t \nu$ for $t \in[0,1]$.

Remark 2.2.1. In [Lit94] and [Lit95], paths in $\mathbb{P}$ are considered modulo reparametrization. However, in this paper, we do not consider paths in $\mathbb{P}$ modulo reparametrization since there is no need to do so. Note that all results of [Lit94] and [Lit95] that we need in this paper still hold in this setting.

Let $\pi \in \mathbb{P}$. A pair $(\underline{\nu} ; \underline{\sigma})$ of a sequence $\underline{\nu}: \nu_{1}, \nu_{2}, \ldots, \nu_{s}$ of elements of $\mathfrak{h}_{\mathbb{R}}^{*}$ and a sequence $\underline{\sigma}: 0=\sigma_{0}<\sigma_{1}<\cdots<\sigma_{s}=1$ of rational numbers is called an expression of $\pi \in \mathbb{P}$ if the following equation holds:

$$
\pi(t)=\sum_{u^{\prime}=1}^{u-1}\left(\sigma_{u^{\prime}}-\sigma_{u^{\prime}-1}\right) \nu_{u^{\prime}}+\left(t-\sigma_{u-1}\right) \nu_{u} \quad \text { for } \sigma_{u-1} \leqslant t \leqslant \sigma_{u}, 1 \leqslant u \leqslant s .
$$

In this case, we write $\pi=(\underline{\nu} ; \underline{\sigma})$. An expression $\left(\nu_{1}, \nu_{2}, \ldots, \nu_{s} ; \underline{\sigma}\right)$ of $\pi$ is said to be reduced if $\nu_{u} \neq \nu_{u+1}$ for any $u=1,2, \ldots, s-1$.

Remark 2.2.2 (see [NS08, Remark 2.5.2]). Let $\pi \in \mathbb{P}$. We easily see that there exists a unique reduced expression of $\pi$. Also, if $\left(\nu_{1}, \nu_{2}, \ldots, \nu_{s} ; \sigma_{0}, \sigma_{1}, \ldots, \sigma_{s}\right)$ is an expression of $\pi$, then the reduced expression of $\pi$ is obtained from this expression by 'omitting' the element $\nu_{u+1}$ such that $\nu_{u}=\nu_{u+1}$ and the corresponding rational number $\sigma_{u}$.

Definition 2.2.3. Let $\pi=\left(\nu_{1}, \nu_{2}, \ldots, \nu_{s} ; \underline{\sigma}\right)$ be an expression of $\pi \in \mathbb{P}$. We call $\nu_{1} \in \mathfrak{h}_{\mathbb{R}}^{*}$ (respectively, $\nu_{s} \in \mathfrak{h}_{\mathbb{R}}^{*}$ ) the initial (respectively, final) direction of $\pi$; it is easy to check that these elements $\nu_{1}, \nu_{s} \in \mathfrak{h}_{\mathbb{R}}^{*}$ do not depend on the choice of an expression of $\pi$. The initial (respectively, final) direction of $\pi$ is denoted by $\iota(\pi)$ (respectively, $\kappa(\pi)$ ).

Remark 2.2.4. Let $\pi_{1}, \pi_{2} \in \mathbb{P}$. We easily see that $\iota\left(\pi_{1} \pm \pi_{2}\right)=\iota\left(\pi_{1}\right) \pm \iota\left(\pi_{2}\right)$ and $\kappa\left(\pi_{1} \pm \pi_{2}\right)=$ $\kappa\left(\pi_{1}\right) \pm \kappa\left(\pi_{2}\right)$. 


\section{S. NAito AND D. SAGAKI}

Let $\lambda \in P$ be an integral weight. Recall from [NS08, Definition 2.6.1] (see also [Lit95, $\S 4]$ ) that a Lakshmibai-Seshadri (LS) path of shape $\lambda$ is a path $\pi \in \mathbb{P}$ having an expression of the form $\pi=\left(\nu_{1}, \nu_{2}, \ldots, \nu_{s} ; \sigma_{0}, \sigma_{1}, \ldots, \sigma_{s}\right)$, where $\nu_{1}, \nu_{2}, \ldots, \nu_{s} \in W \lambda$, and where, for each $1 \leqslant u \leqslant s-1$, there exists a ' $\sigma_{u}$-chain' for $\left(\nu_{u}, \nu_{u+1}\right)$ (see [Lit95, $\S 4$ ] and [NS08, Definition 2.4.5] for the definition of ' $\sigma_{u}$-chain'). We denote by $\mathbb{B}(\lambda)$ the set of all LS paths of shape $\lambda$.

Remark 2.2.5 (see [NS08, Remark 2.6.2]). Let $\lambda \in P$ be an integral weight. The straight line path $\pi_{\nu}(t):=t \nu, t \in[0,1]$, is an element of $\mathbb{B}(\lambda)$ for all $\nu \in W \lambda$.

Lemma 2.2.6. Let $\lambda \in P_{+}^{0}$. If $\pi \in \mathbb{B}(\lambda)$, then $\pi+\pi_{k d_{\lambda} \delta} \in \mathbb{B}(\lambda)$ for all $k \in \mathbb{Z}$.

Proof. Let $k \in \mathbb{Z}$. We know from [NS08, Lemma 2.7.4] that $\pi+\pi_{k d_{\lambda} \delta} \in \mathbb{B}\left(\lambda+k d_{\lambda} \delta\right)$. Also, because there exists $w \in W$ such that $w(\lambda)=\lambda+k d_{\lambda} \delta$ by the definition of $d_{\lambda}$, we deduce from the definition of LS paths that $\mathbb{B}\left(\lambda+k d_{\lambda} \delta\right)=\mathbb{B}(w(\lambda))=\mathbb{B}(\lambda)$ (see [NS08, Remark 2.6.3(3)]). This proves the lemma.

Throughout this paper, we use standard notation and terminology of the theory of (abstract) crystals without further mention; we refer the reader to [Kas95, § 7] and [HK02, § 4.5] for details.

Now, we recall from [Lit94, $\S \S 1.2$ and 1.3] and [Lit95, $\S 1$ ] the definition of the root operators $e_{j}$ and $f_{j}, j \in I$, on $\mathbb{B}(\lambda)$; see also [GL04, $\S 5.1$ ] for the presentation of the definition in this form. Let $\pi \in \mathbb{B}(\lambda)$, and $j \in I$. We set

$$
H_{j}^{\pi}(t):=(\pi(t))\left(h_{j}\right) \quad \text { for } t \in[0,1], \quad m_{j}^{\pi}:=\min \left\{H_{j}^{\pi}(t) \mid t \in[0,1]\right\} .
$$

Then we define $e_{j} \pi$ as follows (note that $m_{j}^{\pi} \in \mathbb{Z}_{\leqslant 0}$ by [Lit95, Lemma $4.5 \mathrm{~d}$ )]). If $m_{j}^{\pi}=0$, then $e_{j} \pi:=\mathbf{0}$, where the $\mathbf{0}$ is an additional element corresponding to ' 0 ' in the theory of crystals. If $m_{j}^{\pi} \leqslant-1$, then we define $e_{j} \pi \in \mathbb{P}$ by

$$
\left(e_{j} \pi\right)(t)= \begin{cases}\pi(t) & \text { if } 0 \leqslant t \leqslant t_{0} \\ \pi\left(t_{0}\right)+r_{j}\left(\pi(t)-\pi\left(t_{0}\right)\right) & \text { if } t_{0} \leqslant t \leqslant t_{1} \\ \pi(t)+\alpha_{j} & \text { if } t_{1} \leqslant t \leqslant 1\end{cases}
$$

where we set

$$
\begin{aligned}
& t_{1}:=\min \left\{t \in[0,1] \mid H_{j}^{\pi}(t)=m_{j}^{\pi}\right\}, \\
& t_{0}:=\max \left\{t \in\left[0, t_{1}\right] \mid H_{j}^{\pi}(t)=m_{j}^{\pi}+1\right\} .
\end{aligned}
$$

Similarly, $f_{j} \pi \in \mathbb{P} \cup\{\mathbf{0}\}$ is defined as follows (note that $H_{j}^{\pi}(1)-m_{j}^{\pi} \in \mathbb{Z}_{\geqslant 0}$ by [Lit95, Lemma 4.5d)] and $\pi(1) \in P$ ). If $H_{j}^{\pi}(1)-m_{j}^{\pi}=0$, then $f_{j} \pi:=\mathbf{0}$. If $H_{j}^{\pi}(1)-m_{j}^{\pi} \geqslant 1$, then we define $f_{j} \pi \in \mathbb{P}$ by

$$
\left(f_{j} \pi\right)(t)= \begin{cases}\pi(t) & \text { if } 0 \leqslant t \leqslant t_{0} \\ \pi\left(t_{0}\right)+r_{j}\left(\pi(t)-\pi\left(t_{0}\right)\right) & \text { if } t_{0} \leqslant t \leqslant t_{1} \\ \pi(t)-\alpha_{j} & \text { if } t_{1} \leqslant t \leqslant 1\end{cases}
$$

where we set

$$
\begin{aligned}
& t_{0}:=\max \left\{t \in[0,1] \mid H_{j}^{\pi}(t)=m_{j}^{\pi}\right\}, \\
& t_{1}:=\min \left\{t \in\left[t_{0}, 1\right] \mid H_{j}^{\pi}(t)=m_{j}^{\pi}+1\right\} .
\end{aligned}
$$




\section{LS PATHS OF LEVEL-ZERO SHAPE AND 1DSUMS}

Using the root operators $e_{j}$ and $f_{j}, j \in I$, we can endow the set $\mathbb{B}(\lambda)$ of all LS paths of shape $\lambda \in P$ with the structure of a ( $P$-weighted) crystal, i.e. the structure of a crystal associated to the Cartan datum $\left(A, \Pi, \Pi^{\vee}, P, P^{\vee}\right)$ (see [Lit95, $\S \S 2$ and 4] and also [NS06, Theorems 1.2.3 and 1.4.5]). In fact, it follows from [Lit95, Lemma 2.1c)] that

$$
\begin{aligned}
-m_{j}^{\pi} & =\max \left\{l \in \mathbb{Z}_{\geqslant 0} \mid e_{j}^{l} \pi \neq \mathbf{0}\right\}, \\
H_{j}^{\pi}(1)-m_{j}^{\pi} & =\max \left\{l \in \mathbb{Z}_{\geqslant 0} \mid f_{j}^{l} \pi \neq \mathbf{0}\right\},
\end{aligned}
$$

and hence that $\varepsilon_{j}(\pi)=-m_{j}^{\pi}$ and $\varphi_{j}(\pi)=H_{j}^{\pi}(1)-m_{j}^{\pi}$.

Let $\lambda \in P$ be an integral weight. For $\pi \in \mathbb{B}(\lambda)$, define a piecewise linear, continuous map $\operatorname{cl}(\pi):[0,1] \rightarrow \mathfrak{h}_{\mathbb{R}}^{*} / \mathbb{R} \delta$ by $(\operatorname{cl}(\pi))(t)=\operatorname{cl}(\pi(t))$ for $t \in[0,1]$, where $\mathrm{cl}: \mathfrak{h}_{\mathbb{R}}^{*} \rightarrow \mathfrak{h}_{\mathbb{R}}^{*} / \mathbb{R} \delta$ is the canonical projection. We set $\mathbb{B}(\lambda)_{\mathrm{cl}}:=\{\operatorname{cl}(\pi) \mid \pi \in \mathbb{B}(\lambda)\}$.

Remark 2.2.7. We see from Remark 2.2.5 that the straight line path $\eta_{\mu}(t)=t \mu, t \in[0,1]$, is contained in $\mathbb{B}(\lambda)_{\mathrm{cl}}$ for all $\mu \in \operatorname{cl}(W \lambda)=\stackrel{\circ}{W} \operatorname{cl}(\lambda)$.

An expression and a reduced expression of $\eta \in \mathbb{B}(\lambda)_{\mathrm{cl}}$ are defined similarly to those for the case of $\mathbb{B}(\lambda)$. In addition, for $\eta \in \mathbb{B}(\lambda)_{\mathrm{cl}}$, we define the initial and final directions of $\eta$ (which do not depend on the choice of an expression of $\eta$ ) as in Definition 2.2.3, and also denote the initial (respectively, final) direction of $\eta \in \mathbb{B}(\lambda)_{\text {cl }}$ by $\iota(\eta)$ (respectively, $\kappa(\eta)$ ).

Remark 2.2.8. Let $\pi=\left(\nu_{1}, \nu_{2}, \ldots, \nu_{s} ; \underline{\sigma}\right)$ be an expression of $\pi \in \mathbb{B}(\lambda)$. Then, $\operatorname{cl}(\pi) \in \mathbb{B}(\lambda)_{\mathrm{cl}}$ has an expression $\operatorname{cl}(\pi)=\left(\operatorname{cl}\left(\nu_{1}\right), \operatorname{cl}\left(\nu_{2}\right), \ldots, \operatorname{cl}\left(\nu_{s}\right) ; \underline{\sigma}\right)$. Hence it follows that

$$
\left\{\begin{array}{l}
\iota(\operatorname{cl}(\pi))=\operatorname{cl}(\iota(\pi)) \in \operatorname{cl}(W \lambda)=\stackrel{\circ}{W} \operatorname{cl}(\lambda) \\
\kappa(\operatorname{cl}(\pi))=\operatorname{cl}(\kappa(\pi)) \in \operatorname{cl}(W \lambda)=\stackrel{\circ}{W} \operatorname{cl}(\lambda)
\end{array} \quad \text { for every } \pi \in \mathbb{B}(\lambda) .\right.
$$

Let $\eta \in \mathbb{B}(\lambda)_{\mathrm{cl}}$, and $j \in I$. We set $H_{j}^{\eta}(t):=(\eta(t))\left(h_{j}\right), t \in[0,1]$, and define $m_{j}^{\eta}$ to be the minimum value of the function $H_{j}^{\eta}(t)$ in the interval $[0,1]$. It is obvious that, for every $\pi \in \mathbb{B}(\lambda)$ and $j \in I$,

$$
H_{j}^{\mathrm{cl}(\pi)}(t)=H_{j}^{\pi}(t) \text { for all } t \in[0,1] \text {, and hence } m_{j}^{\mathrm{cl}(\pi)}=m_{j}^{\pi} .
$$

Remark 2.2.9. Let $\eta \in \mathbb{B}(\lambda)_{\mathrm{cl}}$, and $j \in I$. We see from [Lit95, Lemma 4.5d)] along with (2.2.9) that $\eta(1) \in P_{\mathrm{cl}}$, and that all local minima of the function $H_{j}^{\eta}(t), t \in[0,1]$, are integers. In particular, the minimum value $m_{j}^{\eta}$ of the function $H_{j}^{\eta}(t)$ in the interval $[0,1]$ is a nonpositive integer, and $H_{j}^{\eta}(1)-m_{j}^{\eta}$ is a nonnegative integer.

We define $e_{j} \eta, f_{j} \eta \in \mathbb{B}(\lambda)_{\mathrm{cl}} \cup\{\mathbf{0}\}$ for $\eta \in \mathbb{B}(\lambda)_{\mathrm{cl}}$ and $j \in I$ in the same way as in the case of $\mathbb{B}(\lambda)$. We easily see from $(2.2 .9)$ that

$$
\operatorname{cl}\left(e_{j} \pi\right)=e_{j} \operatorname{cl}(\pi), \quad \operatorname{cl}\left(f_{j} \pi\right)=f_{j} \operatorname{cl}(\pi) \quad \text { for } \pi \in \mathbb{B}(\lambda) \text { and } j \in I,
$$

where $\operatorname{cl}(\mathbf{0})$ is understood to be $\mathbf{0}$.

Remark 2.2.10. Let $\eta \in \mathbb{B}(\lambda)_{\mathrm{cl}}$, and $j \in I$. It follows from the definition of the root operator $e_{j}$ that, if $e_{j} \eta \neq \mathbf{0}$, then the initial direction $\iota\left(e_{j} \eta\right)$ is equal either to $\iota(\eta)$ or to $r_{j}(\iota(\eta))$.

We know from [NS05, Theorem 2.4 and $\S 3.1]$ that the set $\mathbb{B}(\lambda)_{\mathrm{cl}}$ equipped with the root operators $e_{j}$ and $f_{j}, j \in I$, is a $P_{\mathrm{cl}}$-weighted crystal ( $P_{\mathrm{cl}}$-crystal for short), i.e. a crystal associated to the classical Cartan datum $\left(A, \operatorname{cl}(\Pi), \Pi^{\vee}, P_{\mathrm{cl}}, P_{\mathrm{cl}}^{\vee}\right)$, and that the following equations hold:

$$
\begin{gathered}
-m_{j}^{\eta}=\max \left\{l \in \mathbb{Z}_{\geqslant 0} \mid e_{j}^{l} \eta \neq \mathbf{0}\right\}=\varepsilon_{j}(\eta), \\
H_{j}^{\eta}(1)-m_{j}^{\eta}=\max \left\{l \in \mathbb{Z}_{\geqslant 0} \mid f_{j}^{l} \eta \neq \mathbf{0}\right\}=\varphi_{j}(\eta) .
\end{gathered}
$$

For each $\eta \in \mathbb{B}(\lambda)_{\mathrm{cl}}$ and $j \in I$, we set $e_{j}^{\max } \eta:=e_{j}^{\varepsilon_{j}(\eta)} \eta \in \mathbb{B}(\lambda)_{\mathrm{cl}}$. The proof of the next lemma is similar to that of [Lit94, 5.3 Lemma] (see Remark 2.2.9). 


\section{S. NAITO AND D. SAGAKI}

Lemma 2.2.11. Let $\lambda \in P$ be an integral weight, and let $\eta \in \mathbb{B}(\lambda)_{\mathrm{cl}}, j \in I$.

(1) If the initial direction $\iota(\eta) \in P_{\mathrm{cl}}$ of $\eta$ satisfies $(\iota(\eta))\left(h_{j}\right)<0$, then $e_{j} \eta \neq \mathbf{0}$.

(2) For all $0 \leqslant l \leqslant \varepsilon_{j}(\eta)-1$, we have $\iota\left(e_{j}^{l} \eta\right)=\iota(\eta)$.

(3) If $(\iota(\eta))\left(h_{j}\right) \leqslant 0$, then $\iota\left(e_{j}^{\max } \eta\right)=r_{j}(\iota(\eta))$.

Using Lemma 2.2.11(3), we can show the following lemma by induction on $p$.

Lemma 2.2.12. Let $\lambda \in P$ be an integral weight. Let $\eta \in \mathbb{B}(\lambda)_{\mathrm{cl}}$, and set $\mu:=\iota(\eta) \in P_{\mathrm{cl}}$. If $j_{1}, j_{2}, \ldots, j_{p} \in I$ satisfy the condition that $\left(r_{j_{p^{\prime}}} r_{j_{p^{\prime}-1}} \cdots r_{j_{1}}(\mu)\right)\left(h_{j_{p^{\prime}+1}}\right) \leqslant 0$ for all $p^{\prime}=0,1, \ldots, p-1$, then the initial direction of $e_{j_{p}}^{\max } e_{j_{p-1}}^{\max } \cdots e_{j_{1}}^{\max }(\eta) \in \mathbb{B}(\lambda)_{\mathrm{cl}}$ is equal to $r_{j_{p}} r_{j_{p-1}} \cdots r_{j_{1}}(\mu)$.

Lemma 2.2.13. Let $\lambda \in P$ be an integral weight. Let $\eta \in \mathbb{B}(\lambda)_{\mathrm{cl}}$, and set $\mu:=\kappa(\eta) \in P_{\mathrm{cl}}$. If $j \in I$ is such that $\mu\left(h_{j}\right)>0$, then $f_{j} \eta \neq \mathbf{0}$ holds.

Proof. From the assumption of the lemma, we deduce that $H_{j}^{\pi}(1)-m_{j}^{\pi}>0$. Therefore, it follows from Remark 2.2.9 that $H_{j}^{\pi}(1)-m_{j}^{\pi} \geqslant 1$. Hence, by $(2.2 .12)$, we have $\varphi_{j}(\eta) \geqslant 1$, which implies that $f_{j} \eta \neq \mathbf{0}$. This proves the lemma.

\subsection{Regular crystals and simple crystals}

For a proper subset $J$ of $I$, we set $A_{J}:=\left(a_{i j}\right)_{i, j \in J}, \Pi_{J}:=\left\{\alpha_{j}\right\}_{j \in J} \subset \Pi$, and $\Pi_{J}^{\vee}:=\left\{h_{j}\right\}_{j \in J} \subset \Pi^{\vee}$. When we regard a $P_{\mathrm{cl}}$-crystal $\mathcal{B}$ as a crystal associated to the Cartan datum $\left(A_{J}, \operatorname{cl}\left(\Pi_{J}\right), \Pi_{J}^{\vee}, P_{\mathrm{cl}}, P_{\mathrm{cl}}^{\vee}\right)$, we denote it by $\operatorname{res}_{J} \mathcal{B}$. Also, we denote by $U_{q}^{\prime}(\mathfrak{g})_{J}$ the $\mathbb{Q}(q)$-subalgebra of $U_{q}^{\prime}(\mathfrak{g})$ generated by $x_{j}, y_{j}$, $j \in J$, and $q^{h}, h \in P_{\mathrm{cl}}^{\vee}$. Recall that a $P_{\mathrm{cl}}$-crystal $\mathcal{B}$ is said to be regular if, for every proper subset $J \varsubsetneqq I, \operatorname{res}_{J} \mathcal{B}$ is isomorphic to the crystal base of an integrable $U_{q}^{\prime}(\mathfrak{g})_{J}$-module.

Remark 2.3.1. Let $\lambda \in P$ be a level-zero integral weight. We know from [NS05, Proposition 3.13] that $\mathbb{B}(\lambda)_{\mathrm{cl}}$ is a regular $P_{\mathrm{cl}}$-crystal with finitely many elements.

If $\mathcal{B}$ is a regular $P_{\mathrm{cl}}$-crystal equipped with the Kashiwara operators $e_{j}$ and $f_{j}, j \in I$, then we set $e_{j}^{\max } b:=e_{j}^{\varepsilon_{j}(b)} b \in \mathcal{B}$ for $b \in \mathcal{B}$ and $j \in I$, where $\varepsilon_{j}(b):=\max \left\{l \in \mathbb{Z}_{\geqslant 0} \mid e_{j}^{l} b \neq \mathbf{0}\right\}$. For regular $P_{\text {cl-crystals }} \mathcal{B}_{1}$ and $\mathcal{B}_{2}$, we define the tensor product $P_{\text {cl-crystal }} \mathcal{B}_{1} \otimes \mathcal{B}_{2}$ of $\mathcal{B}_{1}$ and $\mathcal{B}_{2}$ as in $[\operatorname{Kas} 95$, $\S 7.3]$ and [HK02, Definition 4.5.3]; note that $\mathcal{B}_{1} \otimes \mathcal{B}_{2}$ is also a regular $P_{\text {cl-crystal. The next lemma }}$ follows immediately from the tensor product rule for crystals.

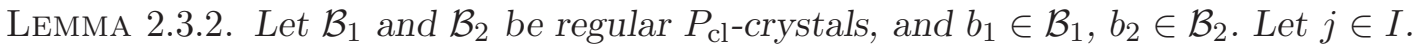

(1) We have $\varepsilon_{j}\left(b_{1} \otimes b_{2}\right) \geqslant \varepsilon_{j}\left(b_{1}\right)$. Therefore, if $e_{j}\left(b_{1} \otimes b_{2}\right)=\mathbf{0}$, then $e_{j} b_{1}=\mathbf{0}$.

(2) Set $L:=\varepsilon_{j}\left(b_{1} \otimes b_{2}\right)$. Then, for $0 \leqslant l \leqslant L$, we have

$$
e_{j}^{l}\left(b_{1} \otimes b_{2}\right)= \begin{cases}b_{1} \otimes e_{j}^{l} b_{2} & \text { if } 0 \leqslant l \leqslant L-\varepsilon_{j}\left(b_{1}\right), \\ e_{j}^{l-L+\varepsilon_{j}\left(b_{1}\right)} b_{1} \otimes e_{j}^{L-\varepsilon_{j}\left(b_{1}\right)} b_{2} & \text { if } L-\varepsilon_{j}\left(b_{1}\right) \leqslant l \leqslant L .\end{cases}
$$

In particular, we have $e_{j}^{\max }\left(b_{1} \otimes b_{2}\right)=e_{j}^{\max } b_{1} \otimes b_{2}^{\prime}$ for some $b_{2}^{\prime} \in \mathcal{B}_{2}$.

Let $\mathcal{B}$ be a regular $P_{\mathrm{cl}^{-}}$-crystal. We define

$$
\|b\|:=\sqrt{(\mathrm{wt}(b), \operatorname{wt}(b))_{\mathrm{cl}}} \quad \text { for } b \in \mathcal{B} .
$$

Lemma 2.3.3. Let $\mathcal{B}$ be a regular $P_{\mathrm{cl}}$-crystal. For each $b \in \mathcal{B}$ and $j \in I$, we have $\left\|e_{j}^{\max } b\right\| \geqslant\|b\|$, with equality if and only if either $e_{j} b=\mathbf{0}$ or $f_{j} b=\mathbf{0}$ holds.

Proof. Using the equation $\varphi_{j}(b)=(\operatorname{wt}(b))\left(h_{j}\right)+\varepsilon_{j}(b)$, we easily see that

$$
\left\|e_{j}^{\max } b\right\|^{2}=\|b\|^{2}+\varepsilon_{j}(b) \varphi_{j}(b)\left(\alpha_{j}, \alpha_{j}\right) .
$$




\section{LS PATHS OF LEVEL-ZERO SHAPE AND 1DSUMS}

Therefore, the inequality $\left\|e_{j}^{\max } b\right\| \geqslant\|b\|$ follows immediately from the fact that $\varepsilon_{j}(b) \geqslant 0, \varphi_{j}(b) \geqslant 0$, and $\left(\alpha_{j}, \alpha_{j}\right)>0$. Also, the equality holds if and only if $\varepsilon_{j}(b)=0$ or $\varphi_{j}(b)=0$, which is equivalent to saying that $e_{j} b=\mathbf{0}$ or $f_{j} b=\mathbf{0}$. This proves the lemma.

Let $\mathcal{B}$ be a regular $P_{\mathrm{cl}}$-crystal. For each $j \in I$, we define $S_{j}: \mathcal{B} \rightarrow \mathcal{B}$ by

$$
S_{j} b= \begin{cases}f_{j}^{l} b & \text { if } l:=(\operatorname{wt} b)\left(h_{j}\right) \geqslant 0, \\ e_{j}^{-l} b & \text { if } l:=(\operatorname{wt} b)\left(h_{j}\right)<0 .\end{cases}
$$

We know from $[\operatorname{Kas} 94, \S 7]$ that there exists a unique action $S: W \rightarrow \operatorname{Bij}(\mathcal{B}), w \mapsto S_{w}$, of the Weyl group $W$ on the set $\mathcal{B}$ such that $S_{r_{j}}=S_{j}$ for all $j \in I$, where $\operatorname{Bij}(\mathcal{B})$ denotes the group of all bijections from the set $\mathcal{B}$ to itself; in fact, if $w=r_{j_{1}} r_{j_{2}} \cdots r_{j_{p}} \in W$ for $j_{1}, j_{2}, \ldots, j_{p} \in I$, then $S_{w}=S_{j_{1}} S_{j_{2}} \cdots S_{j_{p}}$. Note that $\operatorname{wt}\left(S_{w} b\right)=w(\operatorname{wt}(b))$ for all $w \in W$ and $b \in \mathcal{B}$.

Definition 2.3.4 [AK97, $\S 1.4]$. Let $\mathcal{B}$ be a regular $P_{\mathrm{cl}}$-crystal. An element $b \in \mathcal{B}$ is said to be extremal if, for every $w \in W$, either $e_{j} S_{w} b=\mathbf{0}$ or $f_{j} S_{w} b=\mathbf{0}$ holds for each $j \in I$.

Remark 2.3.5. It follows immediately from the definition above that, if $b \in \mathcal{B}$ is an extremal element, then $S_{w} b$ is an extremal element of weight $w(\operatorname{wt}(b))$ for each $w \in W$.

Lemma 2.3.6. Let $\mathcal{B}$ be a regular $P_{\mathrm{cl}}$-crystal with finitely many elements. If $b \in \mathcal{B}$ satisfies the condition that $\|b\|=\max \left\{\left\|b^{\prime}\right\| \mid b^{\prime} \in \mathbb{B}(\lambda)_{\mathrm{cl}}\right\}$, then $b$ is an extremal element.

Proof. Let $w \in W$, and $j \in I$. Since $(\cdot, \cdot)_{\mathrm{cl}}$ is $W$-invariant, it follows that $\left\|S_{w} b\right\|=\|b\|$. Using this, we obtain

$$
\begin{aligned}
\|b\| & \geqslant\left\|e_{j}^{\max } S_{w} b\right\| \quad \text { by the maximality of }\|b\| \\
& \geqslant\left\|S_{w} b\right\| \quad \text { by Lemma } 2.3 .3 \\
& =\|b\|,
\end{aligned}
$$

and hence $\left\|e_{j}^{\max } S_{w} b\right\|=\left\|S_{w} b\right\|$. Therefore, by Lemma 2.3.3, either $e_{j} S_{w} b=\mathbf{0}$ or $f_{j} S_{w} b=\mathbf{0}$ holds. This proves the lemma.

Definition 2.3.7. Let $\mathcal{B}$ be a regular $P_{\mathrm{cl}}$-crystal with finitely many elements. The $P_{\mathrm{cl}}$-crystal $\mathcal{B}$ is said to be simple if it satisfies the following conditions.

(1) The weights of elements of $\mathcal{B}$ are all level-zero.

(2) The set of all extremal elements of $\mathcal{B}$ is exactly the $W$-orbit of some extremal element of $\mathcal{B}$. Also, for each extremal element $b \in \mathcal{B}$, the subset $\mathcal{B}_{\mathrm{wt}(b)} \subset \mathcal{B}$ of all elements of weight wt $(b)$ consists of a single element, i.e. $\mathcal{B}_{\mathrm{wt}(b)}=\{b\}$.

Remark 2.3.8. Let $\mathcal{B}$ be a simple $P_{\mathrm{cl}^{-}}$-crystal. Then it follows from Remark 2.1.5 and Definition 2.3.7(2) that there exists a unique extremal element $b$ of $\mathcal{B}$ such that $\operatorname{wt}(b) \in P_{\mathrm{cl}}$ is level-zero and $I_{0}$-dominant.

Lemma 2.3.9. The following hold.

(1) A simple $P_{\mathrm{cl}}$-crystal is connected.

(2) A tensor product of simple $P_{\mathrm{cl}}$-crystals is also a simple $P_{\mathrm{cl}}$-crystal.

(3) Let $\mathcal{B}_{1}, \mathcal{B}_{2}$ be simple $P_{\mathrm{cl}}$-crystals. Then there exists at most one isomorphism of $P_{\mathrm{cl}}$-crystals from $\mathcal{B}_{1}$ to $\mathcal{B}_{2}$. In particular, any automorphism of a simple $P_{\mathrm{cl}}$-crystal is necessarily the identity map.

Proof. Parts (1) and (2) are precisely Lemmas 1.9 and 1.10 in [AK97], respectively. Let us show part (3). Let $\mathcal{B}_{1}, \mathcal{B}_{2}$ be simple $P_{\mathrm{cl}}$-crystals, and let $\Phi: \mathcal{B}_{1} \stackrel{\sim}{\longrightarrow} \mathcal{B}_{2}$ be an isomorphism of $P_{\mathrm{cl}}$-crystals 


\section{S. NAito AND D. SAGAKI}

from $\mathcal{B}_{1}$ to $\mathcal{B}_{2}$. We see from Remark 2.3.8 that there exists a unique element $b_{1} \in \mathcal{B}_{1}$ (respectively, $\left.b_{2} \in \mathcal{B}_{2}\right)$ such that $b_{1}$ (respectively, $\left.b_{2}\right)$ is extremal, and such that $\operatorname{wt}\left(b_{1}\right)$ (respectively, $\left.\operatorname{wt}\left(b_{2}\right)\right)$ is level-zero and $I_{0}$-dominant. Since $\Phi: \mathcal{B}_{1} \stackrel{\sim}{\longrightarrow} \mathcal{B}_{2}$ is an isomorphism of $P_{\mathrm{cl}}$-crystals, it follows that $\Phi\left(b_{1}\right)$ is extremal, and that $\operatorname{wt}\left(\Phi\left(b_{1}\right)\right)$ is level-zero and $I_{0}$-dominant. Hence, from the uniqueness of such an element, we obtain $\Phi\left(b_{1}\right)=b_{2}$. Also, because a simple $P_{\mathrm{cl}}$-crystal is connected by part (1), an isomorphism of $P_{\mathrm{cl}}$-crystals from $\mathcal{B}_{1}$ to $\mathcal{B}_{2}$ is determined uniquely by the requirement that $\Phi\left(b_{1}\right)=b_{2}$. Thus the proof of the lemma is complete.

\subsection{Tensor product decomposition and combinatorial $\boldsymbol{R}$-matrices}

We know from [NS06, Propositions 3.4.1 and 3.4.2] that, for each $i \in I_{0}$, the $P_{\mathrm{cl}}$-crystal $\mathbb{B}\left(\varpi_{i}\right)_{\mathrm{cl}}$ is a simple $P_{\mathrm{cl}}$-crystal isomorphic to the crystal basis $\mathcal{B}\left(W\left(\varpi_{i}\right)\right)$ of the level-zero fundamental representation $W\left(\varpi_{i}\right)$, which is a finite-dimensional irreducible $U_{q}^{\prime}(\mathfrak{g})$-module introduced in [Kas02, $\S 5.2]$. Because the $W\left(\varpi_{i}\right), i \in I_{0}$, are 'good' $U_{q}^{\prime}(\mathfrak{g})$-modules in the sense of [Kas02, $\S 8$, we deduce from [Kas02, Proposition 10.6] that, for each $i_{1}, i_{2} \in I_{0}$, there exists a unique isomorphism (called a combinatorial $R$-matrix) $R_{\varpi_{i_{1}}, \varpi_{i_{2}}}: \mathbb{B}\left(\varpi_{i_{1}}\right)_{\mathrm{cl}} \otimes \mathbb{B}\left(\varpi_{i_{2}}\right)_{\mathrm{cl}} \stackrel{\sim}{\longrightarrow} \mathbb{B}\left(\varpi_{i_{2}}\right)_{\mathrm{cl}} \otimes \mathbb{B}\left(\varpi_{i_{1}}\right)_{\mathrm{cl}}$ of $P_{\mathrm{cl}}$-crystals (see also [Oka07, § 2.3]); the uniqueness follows from parts (2) and (3) of Lemma 2.3.9. Combining this fact and the tensor product decomposition theorem [NS05, Theorem 3.2], we obtain the following theorem.

Theorem 2.4.1. Let $\mathbf{i}=\left(i_{1}, i_{2}, \ldots, i_{n}\right)$ be an arbitrary sequence of elements of $I_{0}$ (with repetitions allowed), and set $\lambda:=\sum_{k=1}^{n} \varpi_{i_{k}} \in P_{+}^{0}$. Then there exists a unique isomorphism of $P_{\mathrm{cl}}$-crystals,

$$
\Psi_{\mathbf{i}}: \mathbb{B}(\lambda)_{\mathrm{cl}} \stackrel{\sim}{\longrightarrow} \mathbb{B}_{\mathbf{i}}:=\mathbb{B}\left(\varpi_{i_{1}}\right)_{\mathrm{cl}} \otimes \mathbb{B}\left(\varpi_{i_{2}}\right)_{\mathrm{cl}} \otimes \cdots \otimes \mathbb{B}\left(\varpi_{i_{n}}\right)_{\mathrm{cl}} .
$$

Remark 2.4.2. Let $\mathbf{i}=\left(i_{1}, i_{2}, \ldots, i_{n}\right)$ and $\lambda=\sum_{k=1}^{n} \varpi_{i_{k}} \in P_{+}^{0}$ be as in Theorem 2.4.1. It follows from Theorem 2.4.1 along with parts (1) and (2) of Lemma 2.3.9 that $\mathbb{B}(\lambda)_{\mathrm{cl}}$ is a simple $P_{\mathrm{cl}}$-crystal isomorphic to the crystal basis of the tensor product $U_{q}^{\prime}(\mathfrak{g})$-module $W_{\mathbf{i}}:=W\left(\varpi_{i_{1}}\right) \otimes W\left(\varpi_{i_{2}}\right) \otimes \cdots \otimes$ $W\left(\varpi_{i_{n}}\right)$ of the level-zero fundamental representations $W\left(\varpi_{i_{k}}\right), 1 \leqslant k \leqslant n$.

Remark 2.4.3. Let $\lambda \in P_{+}^{0}$. We know from [NS05, Lemma 3.19(1)] that the straight line path $\eta_{\mathrm{cl}(\lambda)}$ is an extremal element of $\mathbb{B}(\lambda)_{\mathrm{cl}}$, and that $S_{w} \eta_{\mathrm{cl}(\lambda)}=\eta_{w \operatorname{cl}(\lambda)}$ for each $w \in W$. Therefore, from Remark 2.4.2, we deduce (recalling the definition of simple $P_{\mathrm{cl}}$-crystals) that each extremal element of $\mathbb{B}(\lambda)_{\mathrm{cl}}$ is a straight line path $\eta_{\mu}$ for some $\mu \in \operatorname{cl}(W \lambda)=\stackrel{\circ}{W} \operatorname{cl}(\lambda)$, and that the number of elements of weight $\mu$ in $\mathbb{B}(\lambda)_{\mathrm{cl}}$ is equal to one for all $\mu \in \operatorname{cl}(W \lambda)=\stackrel{\circ}{W} \operatorname{cl}(\lambda)$. In particular, the straight line path $\eta_{\mathrm{cl}(\lambda)}$ is the unique extremal element of $\mathbb{B}(\lambda)_{\mathrm{cl}}$ whose weight is level-zero and $I_{0}$-dominant, of which we made mention in Remark 2.3.8.

Remark 2.4.4. Let $\lambda \in P_{+}^{0}$. We see from [NS08, Lemma 2.6.4] that the weights of $\mathbb{B}(\lambda)_{\mathrm{cl}}$ are all contained in the set $\operatorname{cl}(\lambda)-a_{0}^{-1} \operatorname{cl}\left(\stackrel{\circ}{Q}_{+}\right)$.

Let $\mathbf{i}=\left(i_{1}, i_{2}, \ldots, i_{n}\right)$ and $\lambda \in P_{+}^{0}$ be as in Theorem 2.4.1. It is easily seen from Remark 2.4.3 and [AK97, Lemma 1.6(1)] that $\eta_{\mathrm{cl}\left(\varpi_{i_{1}}\right)} \otimes \eta_{\mathrm{cl}\left(\varpi_{i_{2}}\right)} \otimes \cdots \otimes \eta_{\mathrm{cl}\left(\varpi_{i_{n}}\right)}$ is the unique extremal element of the simple $P_{\mathrm{cl}}$-crystal $\mathbb{B}_{\mathbf{i}}=\mathbb{B}\left(\varpi_{i_{1}}\right)_{\mathrm{cl}} \otimes \mathbb{B}\left(\varpi_{i_{2}}\right)_{\mathrm{cl}} \otimes \cdots \otimes \mathbb{B}\left(\varpi_{i_{n}}\right)_{\mathrm{cl}}$ whose weight is level-zero and $I_{0}$-dominant. Therefore, we deduce from Remark 2.4.3 and the proof of Lemma 2.3.9(3) that

$$
\Psi_{\mathbf{i}}\left(\eta_{\operatorname{cl}(\lambda)}\right)=\eta_{\operatorname{cl}\left(\varpi_{i_{1}}\right)} \otimes \eta_{\operatorname{cl}\left(\varpi_{i_{2}}\right)} \otimes \cdots \otimes \eta_{\operatorname{cl}\left(\varpi_{i_{n}}\right)} .
$$

Corollary 2.4.5. Let $\lambda, \lambda^{\prime} \in P_{+}^{0}$.

(1) There exists a unique isomorphism $\Psi_{\lambda, \lambda^{\prime}}: \mathbb{B}\left(\lambda+\lambda^{\prime}\right)_{\mathrm{cl}} \stackrel{\sim}{\longrightarrow} \mathbb{B}(\lambda)_{\mathrm{cl}} \otimes \mathbb{B}\left(\lambda^{\prime}\right)_{\mathrm{cl}}$ of $P_{\mathrm{cl}}$-crystals.

(2) There exists a unique isomorphism $R_{\lambda, \lambda^{\prime}}: \mathbb{B}(\lambda)_{\mathrm{cl}} \otimes \mathbb{B}\left(\lambda^{\prime}\right)_{\mathrm{cl}} \stackrel{\sim}{\longrightarrow} \mathbb{B}\left(\lambda^{\prime}\right)_{\mathrm{cl}} \otimes \mathbb{B}(\lambda)_{\mathrm{cl}}$ of $P_{\mathrm{cl}}$-crystals.

Proof. Part (1) follows from Theorem 2.4.1. Part (2) follows immediately from part (1). 


\section{LS PATHS OF LEVEL-ZERO SHAPE AND 1DSUMS}

Let $\lambda, \lambda^{\prime} \in P_{+}^{0}$. By the same reasoning as that yielding (2.4.2), we obtain

$$
\begin{aligned}
\Psi_{\lambda, \lambda^{\prime}}\left(\eta_{\operatorname{cl}\left(\lambda+\lambda^{\prime}\right)}\right) & =\eta_{\operatorname{cl}(\lambda)} \otimes \eta_{\operatorname{cl}\left(\lambda^{\prime}\right)}, \\
R_{\lambda, \lambda^{\prime}}\left(\eta_{\operatorname{cl}(\lambda)} \otimes \eta_{\operatorname{cl}\left(\lambda^{\prime}\right)}\right) & =\eta_{\operatorname{cl}\left(\lambda^{\prime}\right)} \otimes \eta_{\operatorname{cl}(\lambda)} .
\end{aligned}
$$

\subsection{Local energy functions}

Let $\lambda, \lambda^{\prime} \in P_{+}^{0}$, and let $\mathbf{i}=\left(i_{1}, i_{2}, \ldots, i_{n}\right), \mathbf{i}^{\prime}=\left(i_{1}^{\prime}, i_{2}^{\prime}, \ldots, i_{n^{\prime}}^{\prime}\right)$ be sequences of elements of $I_{0}$ such that $\lambda=\sum_{k=1}^{n} \varpi_{i_{k}}$ and $\lambda^{\prime}=\sum_{k^{\prime}=1}^{n^{\prime}} \varpi_{i_{k^{\prime}}}$, respectively. We define the tensor product $U_{q}^{\prime}(\mathfrak{g})$-modules $W_{\mathbf{i}}$ and $W_{\mathbf{i}^{\prime}}$ corresponding to $\mathbf{i}$ and $\mathbf{i}^{\prime}{ }^{\prime}$, respectively, as in Remark 2.4.2; note that both $W_{\mathbf{i}}$ and $W_{\mathbf{i}^{\prime}}$ are 'good' $U_{q}^{\prime}(\mathfrak{g})$-modules (in the sense of [Kas02, §8]) by [Kas02, Proposition 8.7], and that $\mathbb{B}(\lambda)_{\mathrm{cl}}$ and $\mathbb{B}\left(\lambda^{\prime}\right)_{\mathrm{cl}}$ are isomorphic as a $P_{\mathrm{cl}}$-crystal to the crystal bases of $W_{\mathbf{i}}$ and $W_{\mathbf{i}^{\prime}}$, respectively. Therefore, by an argument similar to that in $[\mathrm{Kas} 02, \S 11]$, we obtain the following theorem (see also [Oka07, § 2.3]).

Theorem 2.5.1. Let $\lambda, \lambda^{\prime} \in P_{+}^{0}$. Then, there exists a unique $\mathbb{Z}$-valued function (called a local energy function) $H_{\lambda, \lambda^{\prime}}: \mathbb{B}(\lambda)_{\mathrm{cl}} \otimes \mathbb{B}\left(\lambda^{\prime}\right)_{\mathrm{cl}} \rightarrow \mathbb{Z}$ satisfying the following two conditions.

(H1) For each $\eta_{1} \otimes \eta_{2} \in \mathbb{B}(\lambda)_{\mathrm{cl}} \otimes \mathbb{B}\left(\lambda^{\prime}\right)_{\mathrm{cl}}$ and $j \in I$ such that $e_{j}\left(\eta_{1} \otimes \eta_{2}\right) \neq \mathbf{0}$, the equation

$$
\begin{aligned}
& H_{\lambda, \lambda^{\prime}}\left(e_{j}\left(\eta_{1} \otimes \eta_{2}\right)\right) \\
& \quad= \begin{cases}H_{\lambda, \lambda^{\prime}}\left(\eta_{1} \otimes \eta_{2}\right)+1 & \text { if } j=0, \text { and if } e_{0}\left(\eta_{1} \otimes \eta_{2}\right)=e_{0} \eta_{1} \otimes \eta_{2}, e_{0}\left(\widetilde{\eta}_{2} \otimes \widetilde{\eta}_{1}\right)=e_{0} \widetilde{\eta}_{2} \otimes \widetilde{\eta}_{1}, \\
H_{\lambda, \lambda^{\prime}}\left(\eta_{1} \otimes \eta_{2}\right)-1 & \text { if } j=0, \text { and if } e_{0}\left(\eta_{1} \otimes \eta_{2}\right)=\eta_{1} \otimes e_{0} \eta_{2}, e_{0}\left(\widetilde{\eta}_{2} \otimes \widetilde{\eta}_{1}\right)=\widetilde{\eta}_{2} \otimes e_{0} \widetilde{\eta}_{1}, \\
H_{\lambda, \lambda^{\prime}}\left(\eta_{1} \otimes \eta_{2}\right) & \text { otherwise, }\end{cases}
\end{aligned}
$$

holds, where we set $\widetilde{\eta}_{2} \otimes \widetilde{\eta}_{1}:=R_{\lambda, \lambda^{\prime}}\left(\eta_{1} \otimes \eta_{2}\right) \in \mathbb{B}\left(\lambda^{\prime}\right)_{\mathrm{cl}} \otimes \mathbb{B}(\lambda)_{\mathrm{cl}}$.

(H2) The equation $H_{\lambda, \lambda^{\prime}}\left(\eta_{\operatorname{cl}(\lambda)} \otimes \eta_{\operatorname{cl}\left(\lambda^{\prime}\right)}\right)=0$ holds.

\section{Degree function on LS path crystals}

\subsection{Definition of the degree function}

Let $\lambda \in P_{+}^{0}$. Recall from Lemma 2.1.2 that every element $\nu$ of $W \lambda$ can be written uniquely in the form $\nu=\lambda-\beta+k d_{\lambda} \delta$ with $\beta \in \stackrel{Q}{+}_{+}$and $k \in \mathbb{Z}$.

Lemma 3.1.1. Let $\pi \in \mathbb{B}(\lambda)$. If the initial direction $\iota(\pi) \in W \lambda$ of $\pi$ is contained in $\lambda-\grave{Q}_{+}$, then $\pi(1) \in P$ can be written uniquely in the form $\pi(1)=\lambda-a_{0}^{-1} \beta+a_{0}^{-1} K \delta$ with $\beta \in \stackrel{\circ}{Q}_{+}$and $K \in \mathbb{Z}_{\geqslant 0}$.

Proof. We know from [NS08, Lemma 2.6.4], along with the linear independence of $\alpha_{j}, j \in I$, and $\delta$, that $\pi(1) \in P$ can be written uniquely in the form $\pi(1)=\lambda-a_{0}^{-1} \beta+a_{0}^{-1} K \delta$ with $\beta \in \dot{Q}_{+}$and $K \in \mathbb{Z}$. Let us show that the coefficient $a_{0}^{-1} K$ of $\delta$ in this expression of $\pi(1) \in P$ is nonnegative. Let $\pi=\left(\nu_{1}, \nu_{2}, \ldots, \nu_{s} ; \underline{\sigma}\right)$ be an expression of $\pi \in \mathbb{B}(\lambda)$, and write each $\nu_{u} \in W \lambda$ for $1 \leqslant u \leqslant s$ as $\nu_{u}=\lambda-\beta_{u}+k_{u} d_{\lambda} \delta$, where $\beta_{u} \in \stackrel{Q}{+}_{+}$and $k_{u} \in \mathbb{Z}$ (see Lemma 2.1.2); note that $k_{1}=0$ by the assumption of the lemma. It follows from the definition of LS paths that $\nu_{1} \geqslant \nu_{2} \geqslant \cdots \geqslant \nu_{s}$, and hence from Remark 2.1.3 that $0=k_{1} \leqslant k_{2} \leqslant \cdots \leqslant k_{s}$. Observe by $(2.2 .1)$ that the coefficient $a_{0}^{-1} K$ of $\delta$ in the expression above of $\pi(1) \in P$ is equal to $\sum_{u=1}^{s}\left(\sigma_{u}-\sigma_{u-1}\right) k_{u} d_{\lambda}$. Therefore, we conclude that $a_{0}^{-1} K$ is nonnegative, and hence so is $K$. This proves the lemma.

Let us denote by $\mathbb{B}_{0}(\lambda) \subset \mathbb{B}(\lambda)$ the connected component of the $P$-crystal $\mathbb{B}(\lambda)$ containing the straight line path $\pi_{\lambda}$. We know the following lemma from [NS08, Lemma 4.2.3]. 


\section{S. NAito AND D. SAGAKI}

Lemma 3.1.2. Let $\eta \in \mathbb{B}(\lambda)_{\mathrm{cl}}$. Then, the set $\mathrm{cl}^{-1}(\eta) \cap \mathbb{B}_{0}(\lambda)$ is nonempty, where we set $\mathrm{cl}^{-1}(\eta):=$ $\{\pi \in \mathbb{B}(\lambda) \mid \operatorname{cl}(\pi)=\eta\}$. Moreover, if we take an arbitrary $\pi \in \mathrm{cl}^{-1}(\eta) \cap \mathbb{B}_{0}(\lambda)$, then $\mathrm{cl}^{-1}(\eta) \cap \mathbb{B}_{0}(\lambda)=$ $\left\{\pi+\pi_{k d_{\lambda} \delta} \mid k \in \mathbb{Z}\right\}$.

Proposition 3.1.3. Let $\eta \in \mathbb{B}(\lambda)_{\mathrm{cl}}$. Then, the set $\mathrm{cl}^{-1}(\eta) \cap \mathbb{B}_{0}(\lambda)$ contains a unique element $\pi_{\eta}$ such that $\iota\left(\pi_{\eta}\right) \in \lambda-\dot{Q}_{+}$.

Proof. Let us take $\pi \in \mathrm{cl}^{-1}(\eta) \cap \mathbb{B}_{0}(\lambda)$, and write its initial direction $\iota(\pi) \in W \lambda$ as $\iota(\pi)=\lambda-\beta+k d_{\lambda} \delta$ for $\beta \in \stackrel{Q}{+}_{+}$and $k \in \mathbb{Z}$. Then we see from Lemma 3.1.2 that $\pi_{\eta}:=\pi-\pi_{k d_{\lambda} \delta}$ is also contained in $\mathrm{cl}^{-1}(\eta) \cap \mathbb{B}_{0}(\lambda)$. Also, it is easy to show (see Remark 2.2.4) that $\iota\left(\pi_{\eta}\right)$ is equal to $\iota(\pi)-k d_{\lambda} \delta=\lambda-\beta$, and hence that $\iota\left(\pi_{\eta}\right) \in \lambda-\grave{Q}_{+}$. This proves the existence of $\pi_{\eta}$. The uniqueness of $\pi_{\eta}$ follows immediately from Lemma 3.1.2. This completes the proof of the proposition.

Let $\eta \in \mathbb{B}(\lambda)_{\mathrm{cl}}$, and take $\pi_{\eta} \in \mathrm{cl}^{-1}(\eta) \cap \mathbb{B}_{0}(\lambda)$ of Proposition 3.1.3. Then, by Lemma 3.1.1, we can write $\pi_{\eta}(1) \in P$ in the form $\pi_{\eta}(1)=\lambda-a_{0}^{-1} \beta+a_{0}^{-1} K \delta$ with $\beta \in \dot{Q}_{+}$and $K \in \mathbb{Z}_{\geqslant 0}$. Now, we define the degree $\operatorname{Deg}(\eta) \in \mathbb{Z}_{\leqslant 0}$ of the $\eta \in \mathbb{B}(\lambda)_{\text {cl }}$ by

$$
\operatorname{Deg}(\eta)=-K \text {. }
$$

Proposition 3.1.4. Let $\eta \in \mathbb{B}(\lambda)_{\mathrm{cl}}$. Take $\pi \in \mathrm{cl}^{-1}(\eta) \cap \mathbb{B}(\lambda)$ such that $\iota(\pi) \in \lambda-\stackrel{Q}{+}_{+}$and $\pi \neq \pi_{\eta}$. (1) If we write $\pi(1) \in P$ in the form $\pi(1)=\lambda-a_{0}^{-1} \beta^{\prime}+a_{0}^{-1} K^{\prime} \delta$ with $\beta^{\prime} \in \AA_{+}$and $K^{\prime} \in \mathbb{Z}_{\geqslant 0}$, then we have $-K^{\prime}<\operatorname{Deg}(\eta)$.

(2) If we write the final directions $\kappa\left(\pi_{\eta}\right)$ and $\kappa(\pi)$ of $\pi_{\eta}$ and $\pi$ in the form $\kappa\left(\pi_{\eta}\right)=\lambda-\beta+k d_{\lambda} \delta$ and $\kappa(\pi)=\lambda-\beta^{\prime}+k^{\prime} d_{\lambda} \delta$ with $\beta, \beta^{\prime} \in Q_{+}$and $k, k^{\prime} \in \mathbb{Z}$, respectively, then we have $k<k^{\prime}$.

Remark 3.1.5. Part (1) of Proposition 3.1.4 characterizes the degree $\operatorname{Deg}(\eta) \in \mathbb{Z}_{\leqslant 0}$ of $\eta \in \mathbb{B}(\lambda)_{\mathrm{cl}}$ as the maximum of the nonpositive integers $-K$ for which $\pi(1) \in P$ is of the form $\pi(1)=\lambda-a_{0}^{-1} \beta+$ $a_{0}^{-1} K \delta$ with $\beta \in \stackrel{Q}{+}_{+}$and $K \in \mathbb{Z}_{\geqslant 0}$, where $\pi \in \operatorname{cl}^{-1}(\eta) \cap \mathbb{B}(\lambda)$ is such that $\iota(\pi) \in \lambda-\AA_{+}$. Moreover, the maximum $\operatorname{Deg}(\eta)$ is attained only by $\pi_{\eta} \in \mathrm{cl}^{-1}(\eta) \cap \mathbb{B}_{0}(\lambda)$ of Proposition 3.1.3.

To prove Proposition 3.1.4, we need the following lemma, which can be shown by an argument in the proof of [NS08, Theorem 3.1.1].

LEMma 3.1.6. Each connected component of $\mathbb{B}(\lambda)$ contains a unique element whose reduced expression is of the form

$$
\left(\lambda, \lambda+k_{2} d_{\lambda} \delta, \ldots, \lambda+k_{s} d_{\lambda} \delta ; \sigma_{0}, \sigma_{1}, \ldots, \sigma_{s}\right)
$$

with $k_{2}, \ldots, k_{s} \in \mathbb{Z}$ and $0=\sigma_{0}<\sigma_{1}<\cdots<\sigma_{s}=1$.

Remark 3.1.7. It follows from the definition of LS paths that $\lambda>\lambda+k_{2} d_{\lambda} \delta>\cdots>\lambda+k_{s} d_{\lambda} \delta$. Hence we see from Remark 2.1.3 that $0<k_{2}<\cdots<k_{s}$.

Proof of Proposition 3.1.4. Assume that $\pi \in \mathbb{B}(\lambda)$ lies in a connected component of $\mathbb{B}(\lambda)$ containing an LS path $\pi^{\prime}$ whose reduced expression is of the form (3.1.2). We see from Proposition 3.1.3 and the assumption of the lemma that $\pi$ does not lie in $\mathbb{B}_{0}(\lambda)$, and hence that $s \geqslant 2$.

We set $\psi:=\left(0, k_{2} d_{\lambda} \delta, \ldots, k_{s} d_{\lambda} \delta ; \sigma_{0}, \sigma_{1}, \ldots, \sigma_{s}\right)$; note that $\pi^{\prime}=\pi_{\lambda}+\psi$. Let $X$ be a monomial of $X$ in the root operators $e_{j}, f_{j}$ for $j \in I$ such that $\pi=X \pi^{\prime}$. Then we have

$$
\begin{aligned}
X \pi_{\lambda} & =X\left(\pi^{\prime}-\psi\right)=X \pi^{\prime}-\psi \quad \text { by [NS08, Lemma 2.7.1] } \\
& =\pi-\psi .
\end{aligned}
$$

Since $\operatorname{cl}(\pi)=\eta$, it follows that $\operatorname{cl}\left(X \pi_{\lambda}\right)=\operatorname{cl}(\pi-\psi)=\operatorname{cl}(\pi)=\eta$. Hence we get $X \pi_{\lambda} \in \operatorname{cl}^{-1}(\eta) \cap \mathbb{B}_{0}(\lambda)$. Also, because $\iota(\pi) \in \lambda-\dot{Q}_{+}$and $\iota(\psi)=0$, we see that $\iota\left(X \pi_{\lambda}\right)=\iota(\pi-\psi)=\iota(\pi)-\iota(\psi) \in \lambda-\dot{Q}_{+}$. Hence it follows from Proposition 3.1.3 that $X \pi_{\lambda}=\pi_{\eta}$. Thus we obtain $\pi_{\eta}=\pi-\psi$, and hence

$$
\operatorname{Deg}(\eta)=-K^{\prime}+a_{0} \times(\text { coefficient of } \delta \text { in } \psi(1)) \text { and } k=k^{\prime}-k_{s} \text {. }
$$




\section{LS PATHS OF LEVEL-ZERO SHAPE AND 1DSUMS}

Since $s \geqslant 2$ as seen above, we deduce from Remark 3.1.7 (using (2.2.1)) that the coefficient of $\delta$ in $\psi(1)$ is greater than 0 . Therefore, we conclude that $-K^{\prime}<\operatorname{Deg}(\eta)$. Furthermore, since $k_{s}>0$ with $s \geqslant 2$, it follows that $k<k^{\prime}$. This completes the proof of the proposition.

\subsection{Behavior of the degree function under root operators}

As in $\S 3.1$, let $\lambda \in P_{+}^{0}$.

LEMma 3.2.1. The following hold.

(1) We have $\operatorname{Deg}\left(\eta_{\mathrm{cl}(\lambda)}\right)=0$.

(2) Let $\eta \in \mathbb{B}(\lambda)_{\mathrm{cl}}$, and $j \in I$. If $e_{j} \eta \neq \mathbf{0}$, then (see Remark 2.2.10)

$$
\operatorname{Deg}\left(e_{j} \eta\right)= \begin{cases}\operatorname{Deg}(\eta)-1 & \text { if } j=0 \text { and } \iota\left(e_{0} \eta\right)=\iota(\eta), \\ \operatorname{Deg}(\eta)-(\iota(\eta))\left(h_{0}\right)-1 & \text { if } j=0 \text { and } \iota\left(e_{0} \eta\right)=r_{0}(\iota(\eta)), \\ \operatorname{Deg}(\eta) & \text { if } j \neq 0 .\end{cases}
$$

Proof. Part (1) is obvious from the definition of Deg, since $\pi_{\eta_{\mathrm{cl}(\lambda)}}=\pi_{\lambda}$. Let us prove part (2). It is obvious that $e_{j} \pi_{\eta} \in \mathbb{B}_{0}(\lambda)$ since $\pi_{\eta} \in \mathbb{B}_{0}(\lambda)$ by definition. Also, we know from (2.2.10) that $\operatorname{cl}\left(e_{j} \pi_{\eta}\right)=e_{j} \operatorname{cl}\left(\pi_{\eta}\right)=e_{j} \eta$. Let us write $\pi_{\eta}(1) \in P$ in the form $\pi_{\eta}(1)=\lambda-a_{0}^{-1} \beta-a_{0}^{-1} \operatorname{Deg}(\eta) \delta$ with $\beta \in Q_{+}$.

First, assume that $j \neq 0$. We deduce from Remark 2.2.10 along with Lemma 2.1.2 that $\iota\left(e_{j} \pi_{\eta}\right) \in$ $\lambda-\stackrel{Q}{Q}_{+}$. Because $e_{j} \pi_{\eta} \in \mathbb{B}_{0}(\lambda)$ and $\operatorname{cl}\left(e_{j} \pi_{\eta}\right)=e_{j} \eta$, it follows from Proposition 3.1.3 that $\pi_{e_{j} \eta}=e_{j} \pi_{\eta}$. Since $j \neq 0$, we have

$$
\pi_{e_{j} \eta}(1)=\left(e_{j} \pi_{\eta}\right)(1)=\pi_{\eta}(1)+\alpha_{j}=\lambda-\underbrace{\left(a_{0}^{-1} \beta-\alpha_{j}\right)}_{\in a_{0}^{-1} \stackrel{Q}{+}_{+}}-a_{0}^{-1} \operatorname{Deg}(\eta) \delta,
$$

and hence $\operatorname{Deg}\left(e_{j} \eta\right)=\operatorname{Deg}(\eta)$.

Next, assume that $j=0$ and $\iota\left(e_{0} \eta\right)=\iota(\eta)$. Then we deduce (using (2.2.9)) from the definitions of the root operator $e_{0}$ on $\mathbb{B}(\lambda)$ and the root operator $e_{0}$ on $\mathbb{B}(\lambda)_{\mathrm{cl}}$ that $\iota\left(e_{0} \pi_{\eta}\right)=\iota\left(\pi_{\eta}\right)$, and hence $\iota\left(e_{0} \pi_{\eta}\right) \in \lambda-\stackrel{\circ}{Q}_{+}$. Because $e_{0} \pi_{\eta} \in \mathbb{B}_{0}(\lambda)$ and $\operatorname{cl}\left(e_{0} \pi_{\eta}\right)=e_{0} \eta$, it follows from Proposition 3.1.3 that $\pi_{e_{0} \eta}=e_{0} \pi_{\eta}$. Now, define $\theta \in \dot{Q}_{+}$by $\theta=\delta-a_{0} \alpha_{0}$. Since $\alpha_{0}=a_{0}^{-1}(\delta-\theta)$, we have

$$
\begin{aligned}
\pi_{e_{0} \eta}(1) & =\left(e_{0} \pi_{\eta}\right)(1)=\pi_{\eta}(1)+\alpha_{0} \\
& =\lambda-a_{0}^{-1} \beta-a_{0}^{-1} \operatorname{Deg}(\eta) \delta+a_{0}^{-1}(\delta-\theta) \\
& =\lambda-a_{0}^{-1}(\beta+\theta)-a_{0}^{-1}(\operatorname{Deg}(\eta)-1) \delta,
\end{aligned}
$$

and hence $\operatorname{Deg}\left(e_{0} \eta\right)=\operatorname{Deg}(\eta)-1$.

Finally, assume that $j=0$ and $\iota\left(e_{0} \eta\right)=r_{0}(\iota(\eta))$. Then we deduce (using (2.2.9)) from the definitions of the root operator $e_{0}$ on $\mathbb{B}(\lambda)$ and the root operator on $e_{0} \mathbb{B}(\lambda)_{\mathrm{cl}}$ that

$$
\begin{aligned}
\iota\left(e_{0} \pi_{\eta}\right) & =r_{0} \iota\left(\pi_{\eta}\right)=\iota\left(\pi_{\eta}\right)-\left(\iota\left(\pi_{\eta}\right)\right)\left(h_{0}\right) \alpha_{0} \\
& =\iota\left(\pi_{\eta}\right)+a_{0}^{-1}\left(\iota\left(\pi_{\eta}\right)\right)\left(h_{0}\right) \theta-a_{0}^{-1}\left(\iota\left(\pi_{\eta}\right)\right)\left(h_{0}\right) \delta,
\end{aligned}
$$

where $\theta=\delta-a_{0} \alpha_{0}$ as above. Note that $\iota\left(\pi_{\eta}\right)+a_{0}^{-1}\left(\iota\left(\pi_{\eta}\right)\right)\left(h_{0}\right) \theta \in \lambda-\sum_{j \in I_{0}} \mathbb{Q} \alpha_{j}$, since $\iota\left(\pi_{\eta}\right) \in \lambda-\stackrel{\circ}{+}_{+}$. Because $\iota\left(e_{0} \pi_{\eta}\right) \in W \lambda$, it follows from Lemma 2.1.2 and the linear independence of $\alpha_{j}, j \in I$, and $\delta$ that $\iota\left(\pi_{\eta}\right)+a_{0}^{-1}\left(\iota\left(\pi_{\eta}\right)\right)\left(h_{0}\right) \theta \in \lambda-\stackrel{Q}{Q}_{+}$and $a_{0}^{-1}\left(\iota\left(\pi_{\eta}\right)\right)\left(h_{0}\right) \in \mathbb{Z} d_{\lambda}$. Hence, $a_{0}^{-1}\left(\iota\left(\pi_{\eta}\right)\right)\left(h_{0}\right)=k d_{\lambda}$ for some $k \in \mathbb{Z}$. Also, because $e_{0} \pi_{\eta} \in \mathbb{B}_{0}(\lambda)$ and $\operatorname{cl}\left(e_{0} \pi_{\eta}\right)=e_{0} \eta \in \mathbb{B}_{0}(\lambda)$, we deduce from Lemma 3.1.2 


\section{S. NAITO AND D. SAGAKI}

that $e_{0} \pi_{\eta}+\pi_{k d_{\lambda} \delta} \in \mathrm{cl}^{-1}\left(e_{0} \eta\right) \cap \mathbb{B}_{0}(\lambda)$. Furthermore, we have

$$
\begin{aligned}
\iota\left(e_{0} \pi_{\eta}+\pi_{k d_{\lambda} \delta}\right) & =\iota\left(e_{0} \pi_{\eta}\right)+\iota\left(\pi_{k d_{\lambda} \delta}\right) \quad \text { by Remark } 2.2 .4 \\
& =\iota\left(\pi_{\eta}\right)+a_{0}^{-1}\left(\iota\left(\pi_{\eta}\right)\right)\left(h_{0}\right) \theta-k d_{\lambda} \delta+k d_{\lambda} \delta \quad \text { by }(3.2 .2) \\
& =\iota\left(\pi_{\eta}\right)+a_{0}^{-1}\left(\iota\left(\pi_{\eta}\right)\right)\left(h_{0}\right) \theta,
\end{aligned}
$$

where the last element lies in $\lambda-\stackrel{Q}{+}_{+}$as seen above. Therefore, by Proposition 3.1.3, we get $\pi_{e_{0} \eta}=$ $e_{0} \pi_{\eta}+\pi_{k d_{\lambda} \delta}$. From this, we obtain

$$
\begin{aligned}
\pi_{e_{0} \eta}(1) & =\left(e_{0} \pi_{\eta}\right)(1)+\pi_{k d_{\lambda} \delta}(1)=\pi_{\eta}(1)+\alpha_{0}+k d_{\lambda} \delta \\
& =\lambda-a_{0}^{-1} \beta-a_{0}^{-1} \operatorname{Deg}(\eta) \delta+a_{0}^{-1}(\delta-\theta)+k d_{\lambda} \delta \\
& =\lambda-a_{0}^{-1}(\beta+\theta)-a_{0}^{-1}\left(\operatorname{Deg}(\eta)-a_{0} k d_{\lambda}-1\right) \delta,
\end{aligned}
$$

and hence

$$
\operatorname{Deg}\left(e_{0} \eta\right)=\operatorname{Deg}(\eta)-a_{0} k d_{\lambda}-1=\operatorname{Deg}(\eta)-\left(\iota\left(\pi_{\eta}\right)\right)\left(h_{0}\right)-1 .
$$

Note that $\left(\iota\left(\pi_{\eta}\right)\right)\left(h_{0}\right)=(\iota(\eta))\left(h_{0}\right)$ since $\operatorname{cl}\left(\iota\left(\pi_{\eta}\right)\right)=\iota\left(\operatorname{cl}\left(\pi_{\eta}\right)\right)=\iota(\eta)$ by Remark 2.2.8. Thus we conclude that $\operatorname{Deg}\left(e_{0} \eta\right)=\operatorname{Deg}(\eta)-(\iota(\eta))\left(h_{0}\right)-1$, as desired. This completes the proof of the lemma.

Lemma 3.2.2. Let $\eta \in \mathbb{B}(\lambda)_{\mathrm{cl}}$, and $j \in I$. Assume that $e_{j} \eta \neq \mathbf{0}$, and that $(\iota(\eta))\left(h_{j}\right) \leqslant 0$. Then,

$$
\operatorname{Deg}\left(e_{j}^{\max } \eta\right)= \begin{cases}\operatorname{Deg}(\eta)-\varepsilon_{0}(\eta)-(\iota(\eta))\left(h_{0}\right) & \text { if } j=0 \\ \operatorname{Deg}(\eta) & \text { if } j \neq 0 .\end{cases}
$$

Proof. If $j \neq 0$, then it follows immediately from Lemma 3.2.1(2) that $\operatorname{Deg}\left(e_{j}^{\max } \eta\right)=\operatorname{Deg}(\eta)$. Now assume that $j=0$. If $\varepsilon_{0}(\eta)=0$, i.e. $e_{0} \eta=\mathbf{0}$, then we see from Lemma 2.2.11(1) that $(\iota(\eta))\left(h_{0}\right) \geqslant 0$, which, when combined with the assumption of the lemma, implies that $(\iota(\eta))\left(h_{0}\right)=0$. Hence we have

$$
\operatorname{Deg}\left(e_{0}^{\max } \eta\right)=\operatorname{Deg}\left(e_{0}^{0} \eta\right)=\operatorname{Deg}(\eta)=\operatorname{Deg}(\eta)-\underbrace{\varepsilon_{0}(\eta)}_{=0}-\underbrace{(\iota(\eta))\left(h_{0}\right)}_{=0} .
$$

Thus it remains to consider the case $\varepsilon_{0}(\eta) \geqslant 1$. From Lemmas 2.2.11(2) and 3.2.1(2), it follows that

$$
\operatorname{Deg}\left(e_{0}^{\varepsilon_{0}(\eta)-1} \eta\right)=\operatorname{Deg}(\eta)-\varepsilon_{0}(\eta)+1 .
$$

Therefore, by using parts (2) and (3) of Lemma 2.2.11, we see from Lemma 3.2.1(2) that

$$
\begin{aligned}
\operatorname{Deg}\left(e_{0}^{\max } \eta\right) & =\operatorname{Deg}\left(e_{0}^{\varepsilon_{0}(\eta)} \eta\right)=\operatorname{Deg}\left(e_{0} e_{0}^{\varepsilon_{0}(\eta)-1} \eta\right) \\
& =\operatorname{Deg}\left(e_{0}^{\varepsilon_{0}(\eta)-1} \eta\right)-(\iota(\eta))\left(h_{0}\right)-1 \\
& =\operatorname{Deg}(\eta)-\varepsilon_{0}(\eta)+1-(\iota(\eta))\left(h_{0}\right)-1 \quad \text { by }(3.2 .4) \\
& =\operatorname{Deg}(\eta)-\varepsilon_{0}(\eta)-(\iota(\eta))\left(h_{0}\right) .
\end{aligned}
$$

This proves the lemma.

\section{Relation between the energy function and the degree function}

\subsection{Main results}

Let $\mathbf{i}=\left(i_{1}, i_{2}, \ldots, i_{n}\right)$ be an arbitrary sequence of elements of $I_{0}$, and define the tensor product $P_{\mathrm{cl}}$-crystal $\mathbb{B}_{\mathbf{i}}:=\mathbb{B}\left(\varpi_{i_{1}}\right)_{\mathrm{cl}} \otimes \mathbb{B}\left(\varpi_{i_{2}}\right)_{\mathrm{cl}} \otimes \cdots \otimes \mathbb{B}\left(\varpi_{i_{n}}\right)_{\mathrm{cl}}$. For an element $\eta_{1} \otimes \eta_{2} \otimes \cdots \otimes \eta_{n} \in \mathbb{B}_{\mathbf{i}}$, we define $\eta_{l}^{(k)} \in \mathbb{B}\left(\varpi_{i_{l}}\right)_{\mathrm{cl}}, 1 \leqslant k<l \leqslant n$, as follows (see [HKOTY99, §3] and [HKOTT02, § 3.3]). There 


\section{LS PATHS OF LEVEL-ZERO SHAPE AND 1DSUMS}

exists a unique isomorphism

$$
\begin{aligned}
& \mathbb{B}\left(\varpi_{i_{k}}\right)_{\mathrm{cl}} \otimes \mathbb{B}\left(\varpi_{i_{k+1}}\right)_{\mathrm{cl}} \otimes \cdots \otimes \mathbb{B}\left(\varpi_{i_{l-1}}\right)_{\mathrm{cl}} \otimes \mathbb{B}\left(\varpi_{i_{l}}\right)_{\mathrm{cl}} \\
& \quad \stackrel{\sim}{\longrightarrow} \mathbb{B}\left(\varpi_{i_{l}}\right)_{\mathrm{cl}} \otimes \mathbb{B}\left(\varpi_{i_{k}}\right)_{\mathrm{cl}} \otimes \cdots \otimes \mathbb{B}\left(\varpi_{i_{l-2}}\right)_{\mathrm{cl}} \otimes \mathbb{B}\left(\varpi_{i_{l-1}}\right)_{\mathrm{cl}}
\end{aligned}
$$

of $P_{\mathrm{cl}}$-crystals, which is given as the composition $R_{\varpi_{i_{k}}, \varpi_{i_{l}}} \circ R_{\varpi_{i_{k+1}}, \varpi_{i_{l}}} \circ \cdots \circ R_{\varpi_{i_{l-1}}, \varpi_{i_{l}}}$ of combinatorial $R$-matrices (see $\S 2.4$ ); for uniqueness, see Lemma 2.3.9(3). We define $\eta_{l}^{(k)}$ to be the first factor (which lies in $\left.\mathbb{B}\left(\varpi_{i_{l}}\right)_{\mathrm{cl}}\right)$ of the image of $\eta_{k} \otimes \eta_{k+1} \otimes \cdots \otimes \eta_{l} \in \mathbb{B}\left(\varpi_{i_{k}}\right)_{\mathrm{cl}} \otimes \mathbb{B}\left(\varpi_{i_{k+1}}\right)_{\mathrm{cl}} \otimes \cdots \otimes \mathbb{B}\left(\varpi_{i_{l}}\right)_{\mathrm{cl}}$

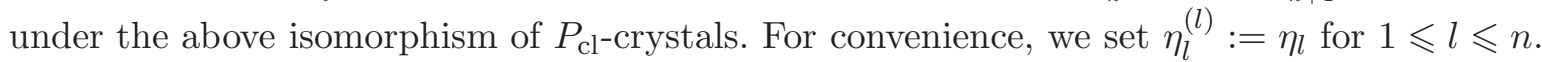

For each $1 \leqslant k \leqslant n$, take (and fix) an arbitrary element $\eta_{k}^{b} \in \mathbb{B}\left(\varpi_{i_{k}}\right)_{\mathrm{cl}}$ such that $f_{j} \eta_{k}^{b}=\mathbf{0}$ for all $j \in I_{0}$. Note that such an element $\eta_{k}^{b} \in \mathbb{B}\left(\varpi_{i_{k}}\right)_{\text {cl }}$ actually exists. Indeed, for each $i \in I_{0}$, we know from Remark 2.2.7 that $\eta_{\widetilde{\varpi}_{i}} \in \mathbb{B}\left(\varpi_{i}\right)_{\mathrm{cl}}$, where $\widetilde{\varpi}_{i}:=w_{0} \operatorname{cl}\left(\varpi_{i}\right) \in P_{\mathrm{cl}}$ (see also Remark 2.1.5); it follows immediately from the definition of the root operators $f_{j}, j \in I_{0}$, that $f_{j} \eta_{\widetilde{\varpi}_{i}}=\mathbf{0}$ for all $j \in I_{0}$.

Now, following [HKOTY99, $\S 3]$ and [HKOTT02, $\S 3.3]$, we define the energy function $D_{\mathbf{i}}: \mathbb{B}_{\mathbf{i}}=$ $\mathbb{B}\left(\varpi_{i_{1}}\right)_{\mathrm{cl}} \otimes \mathbb{B}\left(\varpi_{i_{2}}\right)_{\mathrm{cl}} \otimes \cdots \otimes \mathbb{B}\left(\varpi_{i_{n}}\right)_{\mathrm{cl}} \rightarrow \mathbb{Z}$ by

$$
\begin{aligned}
D_{\mathbf{i}} & \left(\eta_{1} \otimes \eta_{2} \otimes \cdots \otimes \eta_{n}\right) \\
\quad & =\sum_{1 \leqslant k<l \leqslant n} H_{\varpi_{i_{k}}, \varpi_{i_{l}}}\left(\eta_{k} \otimes \eta_{l}^{(k+1)}\right)+\sum_{k=1}^{n} H_{\varpi_{i_{k}}, \varpi_{i_{k}}}\left(\eta_{k}^{b} \otimes \eta_{k}^{(1)}\right) .
\end{aligned}
$$

Also, we define a constant $D_{\mathbf{i}}^{\text {ext }} \in \mathbb{Z}$ by

$$
D_{\mathbf{i}}^{\text {ext }}=\sum_{k=1}^{n} H_{\varpi_{i_{k}}, \varpi_{i_{k}}}\left(\eta_{k}^{b} \otimes \eta_{\mathrm{cl}\left(\varpi_{i_{k}}\right)}\right) .
$$

The main result of this paper is the following theorem.

TheOREM 4.1.1. Let $\mathbf{i}=\left(i_{1}, i_{2}, \ldots, i_{n}\right)$ be an arbitrary sequence of elements of $I_{0}$, and set $\lambda:=$ $\sum_{k=1}^{n} \varpi_{i_{k}} \in P_{+}^{0}$. Then, for every $\eta \in \mathbb{B}(\lambda)_{\mathrm{cl}}$, the equation

$$
\operatorname{Deg}(\eta)=D_{\mathbf{i}}\left(\Psi_{\mathbf{i}}(\eta)\right)-D_{\mathbf{i}}^{\text {ext }}
$$

holds, where $\Psi_{\mathbf{i}}: \mathbb{B}(\lambda)_{\mathrm{cl}} \stackrel{\sim}{\longrightarrow} \mathbb{B}_{\mathbf{i}}$ is the isomorphism of $P_{\mathrm{cl}}$-crystals in Theorem 2.4.1.

We will establish Theorem 4.1.1 under the following plan. First, in $\S 4.2$, we show some technical lemmas needed in subsections that follow. Next, in $\S 4.3$, using these lemmas, we prove Proposition 4.3.1, which is the key to our proof (in $\S 4.4$ ) of Theorem 4.1.2 below. Finally, in $\S 4.5$, we prove Theorem 4.1.3 below, which, when combined with Theorem 4.1.2, establishes Theorem 4.1.1.

THEOREM 4.1.2. Let $\mathbf{i}=\left(i_{1}, i_{2}, \ldots, i_{n}\right)$ be an arbitrary sequence of elements of $I_{0}$, and set $\lambda:=$ $\sum_{k=1}^{n} \varpi_{i_{k}} \in P_{+}^{0}$. Let $\eta \in \mathbb{B}(\lambda)_{\mathrm{cl}}$, and set $\Psi_{\mathbf{i}}(\eta):=\eta_{1} \otimes \eta_{2} \otimes \cdots \otimes \eta_{n} \in \mathbb{B}_{\mathbf{i}}=\mathbb{B}\left(\varpi_{i_{1}}\right)_{\mathrm{cl}} \otimes \mathbb{B}\left(\varpi_{i_{2}}\right)_{\mathrm{cl}} \otimes$ $\cdots \otimes \mathbb{B}\left(\varpi_{i_{n}}\right)_{\mathrm{cl}}$. Then, the following equation holds:

$$
\operatorname{Deg}(\eta)=\sum_{1 \leqslant k<l \leqslant n} H_{\varpi_{i_{k}}, \varpi_{i_{l}}}\left(\eta_{k} \otimes \eta_{l}^{(k+1)}\right)+\sum_{k=1}^{n} \operatorname{Deg}\left(\eta_{k}^{(1)}\right) .
$$

Theorem 4.1.3. Let $i \in I_{0}$, and let $\eta^{b} \in \mathbb{B}\left(\varpi_{i}\right)_{\mathrm{cl}}$ be an element of $\mathbb{B}\left(\varpi_{i}\right)_{\mathrm{cl}}$ such that $f_{j} \eta^{b}=\mathbf{0}$ for all $j \in I_{0}$. Then, for every $\eta \in \mathbb{B}\left(\varpi_{i}\right)_{\mathrm{cl}}$, the following equation holds:

$$
\operatorname{Deg}(\eta)=H_{\varpi_{i}, \varpi_{i}}\left(\eta^{b} \otimes \eta\right)-H_{\varpi_{i}, \varpi_{i}}\left(\eta^{b} \otimes \eta_{\mathrm{cl}\left(\varpi_{i}\right)}\right) .
$$

Remark 4.1.4. Assume that $\mathfrak{g}$ is a nontwisted affine Lie algebra (note that $a_{0}=1$ ), and let $i \in I_{0}$, $m \in \mathbb{Z}_{\geqslant 1}$. In [GL04, $\S 5.7$ and Proposition 5.9], they constructed an isomorphism $\psi$ of $P$-weighted 


\section{S. NAito AND D. SAGAKI}

crystals from the affinization of the $P_{\mathrm{cl}}$-crystal $\left(\mathbb{B}\left(\varpi_{i}\right)_{\mathrm{cl}}\right)^{\otimes m}$ onto $\bigsqcup_{0 \leqslant M<m} \mathbb{B}_{0}\left(m \varpi_{i}+M \delta\right)$; see [GL04, $\S 2.8]$ and [NS08, $\S 4.1]$ for the affinization of a $P_{\mathrm{cl}}$-crystal. Also, in [NS08, §4.2], we constructed an isomorphism $\Theta$ of $P$-weighted crystals from the affinization of the $P_{\mathrm{cl}}$-crystal $\mathbb{B}\left(m \varpi_{i}\right)_{\mathrm{cl}}$ onto $\bigsqcup_{0 \leqslant M<m} \mathbb{B}_{0}\left(m \varpi_{i}+M \delta\right)$. In fact, we can show, without making use of Theorem 4.1.1 (cf. the proof of [NS08, Proposition 4.14]), that the two isomorphisms $\psi$ and $\Theta$ can be considered as identical under the identification $\mathbb{B}\left(m \varpi_{i}\right)_{\mathrm{cl}} \stackrel{\sim}{\rightarrow}\left(\mathbb{B}\left(\varpi_{i}\right)_{\mathrm{cl}}\right)^{\otimes m}$ of $P_{\mathrm{cl}}$-crystals given by Theorem 2.4.1. From this, using the explicit constructions of $\psi$ and $\Theta$ (see the proof of [NS08, Proposition 4.14]), we obtain a description of $\operatorname{Deg}(\eta)$ for $\eta \in \mathbb{B}\left(m \varpi_{i}\right)_{\mathrm{cl}}$ in terms of local energy functions. This description of the degree function Deg $: \mathbb{B}(\lambda)_{\mathrm{cl}} \rightarrow \mathbb{Z}$ is similar and closely related to the one in Theorem 4.1.1 for the special case that $\mathfrak{g}$ is a nontwisted affine Lie algebra and $\lambda=m \varpi_{i}$.

\subsection{Some technical lemmas}

Recall from [Kac90, Proposition 6.3] that a real root of $\mathfrak{g}$ lies either in $a_{0}^{-1} \stackrel{Q}{Q}_{+}+a_{0}^{-1} \mathbb{Z} \delta$ or in $-a_{0}^{-1} \stackrel{\circ}{Q}_{+}+a_{0}^{-1} \mathbb{Z} \delta$.

Lemma 4.2.1. Let $\lambda \in P_{+}^{0}$, and let $w \in W, j \in I$.

(1) If $(w(\operatorname{cl}(\lambda)))\left(h_{j}\right)<0$, then the real root $w^{-1}\left(\alpha_{j}\right)$ lies in $-a_{0}^{-1} \stackrel{\circ}{Q}_{+}+a_{0}^{-1} \mathbb{Z} \delta$.

(2) If the real root $w^{-1}\left(\alpha_{j}\right)$ lies in $-a_{0}^{-1} \stackrel{\circ}{Q}_{+}+a_{0}^{-1} \mathbb{Z} \delta$, then $(w(\operatorname{cl}(\lambda)))\left(h_{j}\right) \leqslant 0$.

(3) Assume that $\lambda$ is strictly $I_{0}$-dominant. Then, $(w(\operatorname{cl}(\lambda)))\left(h_{j}\right)<0$ if and only if the real root $w^{-1}\left(\alpha_{j}\right)$ lies in $-a_{0}^{-1} \stackrel{\circ}{+}_{+}+a_{0}^{-1} \mathbb{Z} \delta$.

Proof. Since $\left(\alpha_{j}, \alpha_{j}\right) \in \mathbb{Z}_{>0}$ for all $j \in I$, and since

$$
(w(\operatorname{cl}(\lambda)))\left(h_{j}\right)=(w(\lambda))\left(h_{j}\right)=\frac{2\left(w(\lambda), \alpha_{j}\right)}{\left(\alpha_{j}, \alpha_{j}\right)}=\frac{2\left(\lambda, w^{-1}\left(\alpha_{j}\right)\right)}{\left(\alpha_{j}, \alpha_{j}\right)},
$$

it follows that $(w(\operatorname{cl}(\lambda)))\left(h_{j}\right)<0$ if and only if $\left(\lambda, w^{-1}\left(\alpha_{j}\right)\right)<0$, and that $(w(\operatorname{cl}(\lambda)))$ $\left(h_{j}\right)=0$ if and only if $\left(\lambda, w^{-1}\left(\alpha_{j}\right)\right)=0$. Also, since $\lambda$ is level-zero and $I_{0}$-dominant, and since $\left(\alpha_{j}, \alpha_{j}\right) \in \mathbb{Z}_{>0}$ for all $j \in I_{0}$, we have

$$
\left(\lambda, \pm a_{0}^{-1} \stackrel{\circ}{Q}_{+}+a_{0}^{-1} \mathbb{Z} \delta\right)=\left(\lambda, \pm a_{0}^{-1} \stackrel{\circ}{Q}_{+}\right) \subset \pm \mathbb{Q} \geqslant 0 .
$$

Now, all the assertions of the lemma follow immediately from the discussion above.

Lemma 4.2.2. Let $\lambda \in P_{+}^{0}$, and let $\eta \in \mathbb{B}(\lambda)_{\mathrm{cl}}, j \in I$. Assume that $\eta$ has an expression of the form $\eta=\left(\mu_{1}, \mu_{2} ; 0, \sigma, 1\right)$, with $\mu_{1}, \mu_{2} \in \operatorname{cl}(W \lambda)=\stackrel{\circ}{W} \operatorname{cl}(\lambda)$ and $0<\sigma<1$. If $\mu_{1}\left(h_{j}\right)<0$, then $e_{j}^{\max } \eta=\left(r_{j}\left(\mu_{1}\right), \mu_{2}^{\prime} ; 0, \sigma, 1\right)$, where

$$
\mu_{2}^{\prime}:= \begin{cases}\mu_{2} & \text { if } \mu_{2}\left(h_{j}\right) \geqslant 0 \\ r_{j} \mu_{2} & \text { if } \mu_{2}\left(h_{j}\right)<0 .\end{cases}
$$

Proof. First, assume that $\mu_{2}\left(h_{j}\right) \geqslant 0$. Then, since $\mu_{1}\left(h_{j}\right)<0$ by the assumption of the lemma, it follows that the function $H_{j}^{\eta}(t)$ is strictly decreasing in the interval $[0, \sigma]$, and $m_{j}^{\eta}=H_{j}^{\eta}(\sigma)<0$; note that $\varepsilon_{j}(\eta)=-m_{j}^{\eta}$ by $(2.2 .11)$. For $0 \leqslant l \leqslant \varepsilon_{j}(\eta)=-m_{j}^{\eta}$, let $\sigma^{(l)}$ be the unique point in $[0, \sigma]$ such that $H_{j}^{\eta}\left(\sigma^{(l)}\right)=m_{j}^{\eta}+l$; observe that $0=\sigma^{\left(\varepsilon_{j}(\eta)\right)}<\sigma^{\left(\varepsilon_{j}(\eta)-1\right)}<\cdots<\sigma^{(0)}=\sigma$. Now it is easily shown by induction on $l$ that, for $0 \leqslant l \leqslant \varepsilon_{j}(\eta)$,

$$
\left(e_{j}^{l} \eta\right)(t)= \begin{cases}\eta(t) & \text { if } 0 \leqslant t \leqslant \sigma^{(l)} \\ \eta\left(\sigma^{(l)}\right)+r_{j}\left(\eta(t)-\eta\left(\sigma^{(l)}\right)\right) & \text { if } \sigma^{(l)} \leqslant t \leqslant \sigma \\ \eta(t)+l \alpha_{j} & \text { if } \sigma \leqslant t \leqslant 1\end{cases}
$$




\section{LS PATHS OF LEVEL-ZERO SHAPE AND 1DSUMS}

In particular, by taking $l=\varepsilon_{j}(\eta)$, we have

$$
\left(e_{j}^{\max } \eta\right)(t)= \begin{cases}r_{j}(\eta(t)) & \text { if } 0 \leqslant t \leqslant \sigma \\ \eta(t)+\varepsilon_{j}(\eta) \alpha_{j} & \text { if } \sigma \leqslant t \leqslant 1,\end{cases}
$$

which implies that $e_{j}^{\max } \eta=\left(r_{j}\left(\mu_{1}\right), \mu_{2} ; 0, \sigma, 1\right)$, since $\eta=\left(\mu_{1}, \mu_{2} ; 0, \sigma, 1\right)$.

Next, assume that $\mu_{2}\left(h_{j}\right)<0$. Then, since $\mu_{1}\left(h_{j}\right)<0$ by the assumption of the lemma, it follows that the function $H_{j}^{\eta}(t)$ is strictly decreasing in the interval $[0,1]$, and $m_{j}^{\eta}=H_{j}^{\eta}(1)<0$; note that $\varepsilon_{j}(\eta)=-m_{j}^{\eta}$ by $(2.2 .11)$. For $0 \leqslant l \leqslant \varepsilon_{j}(\eta)=-m_{j}^{\eta}$, let $\sigma^{(l)}$ be the unique point in $[0,1]$ such that $H_{j}^{\eta}\left(\sigma^{(l)}\right)=m_{j}^{\eta}+l$; observe that $0=\sigma^{\left(\varepsilon_{j}(\eta)\right)}<\sigma^{\left(\varepsilon_{j}(\eta)-1\right)}<\cdots<\sigma^{(0)}=1$. Now it is easily shown by induction on $l$ that, for $0 \leqslant l \leqslant \varepsilon_{j}(\eta)$,

$$
\left(e_{j}^{l} \eta\right)(t)= \begin{cases}\eta(t) & \text { if } 0 \leqslant t \leqslant \sigma^{(l)} \\ \eta\left(\sigma^{(l)}\right)+r_{j}\left(\eta(t)-\eta\left(\sigma^{(l)}\right)\right) & \text { if } \sigma^{(l)} \leqslant t \leqslant 1 .\end{cases}
$$

In particular, by taking $l=\varepsilon_{j}(\eta)$, we have

$$
\left(e_{j}^{\max } \eta\right)(t)=r_{j}(\eta(t)) \quad \text { for } t \in[0,1],
$$

which implies that $e_{j}^{\max } \eta=\left(r_{j}\left(\mu_{1}\right), r_{j}\left(\mu_{2}\right) ; 0, \sigma, 1\right)$, since $\eta=\left(\mu_{1}, \mu_{2} ; 0, \sigma, 1\right)$. This completes the proof of the lemma.

Lemma 4.2.3. Let $\lambda \in P_{+}^{0}$. Let $\eta=\left(\mu_{1}, \mu_{2}, \ldots, \mu_{s} ; \sigma_{0}, \sigma_{1}, \sigma_{2}, \ldots, \sigma_{s}\right)$ be an expression of $\eta \in \mathbb{B}(\lambda)_{\mathrm{cl}}$, and assume that $s \geqslant 2$.

(1) The element $\eta^{\prime}:=\left(\mu_{1}, \mu_{2} ; \sigma_{0}, \sigma_{1}, \sigma_{s}\right)$ is contained in $\mathbb{B}(\lambda)_{\mathrm{cl}}$.

(2) If $j \in I$ is such that $\mu_{1}\left(h_{j}\right)<0$, then $\left(e_{j}^{\max } \eta\right)(t)=\left(e_{j}^{\max } \eta^{\prime}\right)(t)$ for all $t \in\left[0, \sigma_{2}\right]$. Hence, by Lemma 4.2.2, $e_{j}^{\max } \eta \in \mathbb{B}(\lambda)_{\mathrm{cl}}$ has an expression of the form

$$
e_{j}^{\max } \eta=\left(r_{j} \mu_{1}, \mu_{2}^{\prime}, \mu_{3}^{\prime}, \ldots, \mu_{s^{\prime}}^{\prime} ; \sigma_{0}, \sigma_{1}, \sigma_{2}, \sigma_{3}^{\prime}, \ldots, \sigma_{s^{\prime}}^{\prime}\right),
$$

where

$$
\mu_{2}^{\prime}= \begin{cases}\mu_{2} & \text { if } \mu_{2}\left(h_{j}\right) \geqslant 0, \\ r_{j} \mu_{2} & \text { if } \mu_{2}\left(h_{j}\right)<0 .\end{cases}
$$

Proof. (1) Let $\pi \in \mathbb{B}(\lambda)$ be such that $\operatorname{cl}(\pi)=\eta$, and let

$$
\pi=\left(\nu_{1}, \nu_{2}, \ldots, \nu_{s^{\prime \prime}} ; \sigma_{0}^{\prime \prime}, \sigma_{1}^{\prime \prime}, \sigma_{2}^{\prime \prime}, \ldots, \sigma_{s^{\prime \prime}}^{\prime \prime}\right)
$$

be an expression of $\pi$. By 'inserting' (see [NS08, Remark 2.5.2(2)]) $\sigma_{1}$ (respectively, $\sigma_{2}$ ) between $\sigma_{k}^{\prime \prime}$ and $\sigma_{k+1}^{\prime \prime}$ such that $\sigma_{k}^{\prime \prime}<\sigma_{1}$ (respectively, $\left.\sigma_{2}\right)<\sigma_{k+1}^{\prime \prime}$ if necessary, we may assume that there exist $1 \leqslant u_{1}<u_{2} \leqslant s^{\prime \prime}$ such that $\sigma_{u_{1}}^{\prime \prime}=\sigma_{1}$ and $\sigma_{u_{2}}^{\prime \prime}=\sigma_{2}$. By Remark 2.2.8 and the condition that $\operatorname{cl}(\pi)=\eta$, we have

$$
\begin{array}{ll}
\operatorname{cl}\left(\nu_{u}\right)=\mu_{1} & \text { for all } 1 \leqslant u \leqslant u_{1}, \\
\operatorname{cl}\left(\nu_{u}\right)=\mu_{2} & \text { for all } u_{1}+1 \leqslant u \leqslant u_{2} .
\end{array}
$$

Set $\pi^{\prime}:=\left(\nu_{1}, \nu_{2}, \ldots, \nu_{u_{2}} ; \sigma_{0}^{\prime \prime}, \sigma_{1}^{\prime \prime}, \ldots, \sigma_{u_{2}-1}^{\prime \prime}, \sigma_{s^{\prime \prime}}^{\prime \prime}\right)$. Then we can easily deduce from the definition of LS paths (see also [Lit95, Lemma 4.5b)]) that $\pi^{\prime} \in \mathbb{B}(\lambda)$. Furthermore, it is clear from Remark 2.2.8 and (4.2.4) that $\operatorname{cl}\left(\pi^{\prime}\right)=\eta^{\prime}$. Thus we have proved that $\eta^{\prime} \in \mathbb{B}(\lambda)_{\mathrm{cl}}$.

(2) For $0 \leqslant l \leqslant \varepsilon_{j}(\eta)-1$, we set $\eta_{l}:=e_{j}^{l} \eta \in \mathbb{B}(\lambda)_{\mathrm{cl}}$, and

$$
t_{1}^{(l)}:=\min \left\{t \in[0,1] \mid H_{j}^{\eta_{l}}(t)=m_{j}^{\eta_{l}}\right\}, \quad t_{0}^{(l)}:=\max \left\{t \in\left[0, t_{1}^{l}\right] \mid H_{j}^{\eta_{l}}(t)=m_{j}^{\eta_{l}}+1\right\} .
$$

By (2.2.11), we have

$$
m_{j}^{\eta_{l}}=-\varepsilon_{j}\left(\eta_{l}\right)=-\varepsilon_{j}\left(e_{j}^{l} \eta\right)=-\varepsilon_{j}(\eta)+l=m_{j}^{\eta}+l \quad \text { for } 0 \leqslant l \leqslant \varepsilon_{j}(\eta)-1 .
$$




\section{S. NAITO AND D. SAGAKI}

Now, let us assume that $\mu_{2}\left(h_{j}\right) \geqslant 0$. Since $\mu_{1}\left(h_{j}\right)<0$ by the assumption of the lemma, it follows that $k_{1}:=H_{j}^{\eta}\left(\sigma_{1}\right)<0$. Also, since $\mu_{2}\left(h_{j}\right) \geqslant 0$, we see that the function $H_{j}^{\eta}(t), t \in[0,1]$, attains a local minimum at $t=\sigma_{1}$. Therefore, we obtain $k_{1} \in \mathbb{Z}_{<0}$ by Remark 2.2.9. Observe that

$$
H_{j}^{\eta}(t) \geqslant k_{1} \quad \text { for all } t \in\left[0, \sigma_{2}\right] .
$$

We set $l_{1}:=k_{1}-m_{j}^{\eta} \in \mathbb{Z}_{\geqslant 0}$; note that $l_{1}<-m_{j}^{\eta}=\varepsilon_{j}(\eta)$ since $k_{1} \in \mathbb{Z}_{<0}$.

Claim. For all $0 \leqslant l \leqslant l_{1}$, we have $\eta_{l}(t)=\eta(t)$ for all $t \in\left[0, \sigma_{2}\right]$.

Proof of Claim. We show the assertion by induction on $l$. When $l=0$, the assertion obviously holds. Assume that $0<l \leqslant l_{1}$ and that $\eta_{l-1}(t)=\eta(t)$ for all $t \in\left[0, \sigma_{2}\right]$. Note that $\eta_{l}=e_{j} \eta_{l-1}$. Therefore, by the definition of the root operator $e_{j}$, it suffices to show that $\sigma_{2} \leqslant t_{0}^{(l-1)}$. We see from (4.2.5) that

$$
H_{j}^{\eta_{l-1}}\left(t_{1}^{(l-1)}\right)=m_{j}^{\eta_{l-1}}=m_{j}^{\eta}+l-1 \leqslant m_{j}^{\eta}+l_{1}-1=k_{1}-1 .
$$

Also, since $\eta_{l-1}(t)=\eta(t)$ for all $t \in\left[0, \sigma_{2}\right]$ by the induction hypothesis, it follows from (4.2.6) that $H_{j}^{\eta_{l-1}}(t)=H_{j}^{\eta}(t) \geqslant k_{1}$ for all $t \in\left[0, \sigma_{2}\right]$. Hence we deduce from (4.2.7) that $t_{1}^{(l-1)} \notin\left[0, \sigma_{2}\right]$, i.e. that $\sigma_{2}<t_{1}^{(l-1)}$.

We have $H_{j}^{\eta_{l-1}}\left(\sigma_{2}\right)=H_{j}^{\eta}\left(\sigma_{2}\right) \geqslant k_{1}=m_{j}^{\eta}+l_{1} \geqslant m_{j}^{\eta}+l=m_{j}^{\eta_{l-1}}+1$ as seen above, and $H_{j}^{\eta_{l-1}}\left(t_{1}^{(l-1)}\right)=m_{j}^{\eta_{l-1}}<m_{j}^{\eta_{l-1}}+1$. Therefore, from the continuity of the function $H_{j}^{\eta_{l-1}}(t)$ in the interval $\sigma_{2} \leqslant t \leqslant t_{1}^{(l-1)}$, we conclude that there exists $\sigma_{2} \leqslant t^{\prime}<t_{1}^{(l-1)}$ such that $H_{j}^{\eta_{l-1}}\left(t^{\prime}\right)=m_{j}^{\eta_{l-1}}+1$. Hence it follows from the definition of $t_{0}^{(l-1)}$ that $t_{0}^{(l-1)} \geqslant t^{\prime} \geqslant \sigma_{2}$. This proves the claim.

From the claim above, by taking $l=l_{1}$, we obtain $\eta_{l_{1}}(t)=\eta(t)$ for all $t \in\left[0, \sigma_{2}\right]$. Consequently, we see from the definition of $t_{1}^{\left(l_{1}\right)}$ that $t_{1}^{\left(l_{1}\right)}=\sigma_{1}$, since $m_{j}^{\eta_{l_{1}}}=m_{j}^{\eta}+l_{1}=k_{1}$ and $H_{j}^{\eta}\left(\sigma_{1}\right)=k_{1}$. Therefore, as in the proof of Lemma 4.2.2, we can show (using $\eta(t)=\eta_{l_{1}}(t)$ for $t \in\left[0, \sigma_{2}\right]$ ) that

$$
\begin{aligned}
\left(e_{j}^{\max } \eta\right)(t)= & \left(e_{j}^{-m_{j}^{\eta}} \eta\right)(t)=\left(e_{j}^{l_{1}-k_{1}} \eta\right)(t)=\left(e_{j}^{-k_{1}} \eta_{l_{1}}\right)(t) \\
= & \begin{cases}r_{j}(\eta(t)) & \text { if } 0 \leqslant t \leqslant \sigma_{1}, \\
\eta(t)-k_{1} \alpha_{j} & \text { if } \sigma_{1} \leqslant t \leqslant \sigma_{2}, \\
\eta_{l_{1}}(t)-k_{1} \alpha_{j} & \text { if } \sigma_{2} \leqslant t \leqslant 1 .\end{cases}
\end{aligned}
$$

From this and Lemma 4.2.2, we conclude that $\left(e_{j}^{\max } \eta\right)(t)=\left(e_{j}^{\max } \eta^{\prime}\right)(t)$ for $t \in\left[0, \sigma_{2}\right]$.

The proof for the case $\mu_{2}\left(h_{j}\right)<0$ is similar; we give only a sketch of the proof. Take the largest $u \in\{2,3, \ldots, s\}$ such that $\mu_{u^{\prime}}\left(h_{j}\right)<0$ for all $1 \leqslant u^{\prime} \leqslant u$. Then we see that the function $H_{j}^{\eta}(t)$, $t \in[0,1]$, attains a local minimum at $t=\sigma_{u}$, and hence that $k_{u}:=H_{j}^{\eta}\left(\sigma_{u}\right) \in \mathbb{Z}_{<0}$ by Remark 2.2.9. We set $l_{u}:=k_{u}-m_{j}^{\eta} \in \mathbb{Z}_{\geqslant 0}$. Exactly in the same way as above, we can show that $\eta(t)=\eta_{l_{u}}(t)$ for all $t \in\left[0, \sigma_{u}\right]$. Consequently, we have $t_{1}^{\left(l_{u}\right)}=\sigma_{u}$, since $\mu_{u^{\prime}}\left(h_{j}\right)<0$ for all $1 \leqslant u^{\prime} \leqslant u$. Therefore, as in the proof of Lemma 4.2.2, we can show (using $\eta(t)=\eta_{l_{u}}(t)$ for $t \in\left[0, \sigma_{u}\right]$ ) that

$$
\left(e_{j}^{\max } \eta\right)(t)=\left(e_{j}^{-k_{u}} \eta_{l_{u}}\right)(t)= \begin{cases}r_{j}(\eta(t)) & \text { if } 0 \leqslant t \leqslant \sigma_{u} \\ \eta_{l_{u}}(t)-k_{u} \alpha_{j} & \text { if } \sigma_{u} \leqslant t \leqslant 1 .\end{cases}
$$

From this and Lemma 4.2.2, we conclude that $\left(e_{j}^{\max } \eta\right)(t)=\left(e_{j}^{\max } \eta^{\prime}\right)(t)$ for $t \in\left[0, \sigma_{2}\right]$. This completes the proof of the lemma.

\subsection{Key proposition to the proof of Theorem 4.1.2}

The following proposition plays a key role in the proof of Theorem 4.1.2. 


\section{LS PATHS OF LEVEL-ZERO SHAPE AND 1DSUMS}

Proposition 4.3.1. Let $\lambda \in P_{+}^{0}$, and let $\eta \in \mathbb{B}(\lambda)_{\mathrm{cl}}$ be an element of $\mathbb{B}(\lambda)_{\mathrm{cl}}$ such that $e_{j} \eta=\mathbf{0}$ for all $j \in I_{0}$. Then, there exists a sequence $j_{1}, j_{2}, \ldots, j_{N} \in I$ such that:
(A) $e_{j_{N}}^{\max } e_{j_{N-1}}^{\max } \cdots e_{j_{1}}^{\max } \eta=\eta_{\mathrm{cl}(\lambda)}$
(B) $r_{j_{1}} r_{j_{2}} \cdots r_{j_{p}}\left(\alpha_{j_{p+1}}\right) \in-a_{0}^{-1} \stackrel{\circ}{Q}_{+}+a_{0}^{-1} \mathbb{Z} \delta$ for $p=0,1, \ldots, N-1$.

In order to prove Proposition 4.3.1, we need Lemmas 4.3.2 and 4.3.3 below.

Lemma 4.3.2. Let $\lambda \in \sum_{i \in I_{0}} \mathbb{Z}_{>0} \varpi_{i}$ be a strictly $I_{0}$-dominant integral weight. Let $\eta$ be an element of $\mathbb{B}(\lambda)_{\mathrm{cl}}$ such that $\iota(\eta)=\operatorname{cl}(\lambda)$. Then, there exists a sequence $j_{1}, j_{2}, \ldots, j_{N} \in I$ such that:

(A) $e_{j_{N}}^{\max } e_{j_{N-1}}^{\max } \cdots e_{j_{1}}^{\max } \eta=\eta_{\mathrm{cl}(\lambda)} ;$
$\left(\mathrm{B}^{\prime}\right)\left(r_{j_{p}} r_{j_{p-1}} \cdots r_{j_{1}}(\mathrm{cl}(\lambda))\right)\left(h_{j_{p+1}}\right)<0$ for $p=0,1, \ldots, N-1$.

Proof. The crucial point in the proof is to consider the special case of $\eta \in \mathbb{B}(\lambda)_{\mathrm{cl}}$ having an expression of the form

$$
\eta=(\operatorname{cl}(\lambda), \mu ; 0, \sigma, 1), \quad \text { with } \mu \in \operatorname{cl}(W \lambda)=\stackrel{\circ}{W} \operatorname{cl}(\lambda) \text { and } 0<\sigma<1,
$$

and prove the assertion of the lemma in this case. Before doing this, we show how to deduce the general case from the special case (4.3.1). By Remark 2.3.1, the set of all elements of [0,1] appearing as the $\sigma_{u}$ in the reduced expression $\left(\underline{\nu} ; \sigma_{0}, \sigma_{1}, \ldots, \sigma_{s}\right)$ of some element of $\mathbb{B}(\lambda)_{\mathrm{cl}}$ is a finite set. Let $\eta=\left(\operatorname{cl}(\lambda), \mu_{2}, \ldots, \mu_{s} ; \sigma_{0}, \sigma_{1}, \sigma_{2}, \ldots, \sigma_{s}\right)$ be the reduced expression of $\eta$. Using the finiteness above, we show the assertion of Lemma 4.3 .2 by descending induction on $\sigma_{1}$ in the reduced expression $\left(\operatorname{cl}(\lambda), \mu_{2}, \ldots, \mu_{s} ; \sigma_{0}, \sigma_{1}, \sigma_{2}, \ldots, \sigma_{s}\right)$ of $\eta \in \mathbb{B}(\lambda)_{\mathrm{cl}}$. When $\sigma_{1}=1$, we have $\eta=\eta_{\mathrm{cl}(\lambda)}$, since $\iota(\eta)=\operatorname{cl}(\lambda)$ by the assumption of the lemma. Thus the assertion obviously holds. Assume that $\sigma_{1}<1$, or equivalently $s \geqslant 2$. Set $\eta^{\prime}:=\left(\operatorname{cl}(\lambda), \mu_{2} ; \sigma_{0}, \sigma_{1}, \sigma_{s}\right)$; note that $\eta^{\prime} \in \mathbb{B}(\lambda)_{\mathrm{cl}}$ by Lemma 4.2.3(1). It follows from the assertion in the special case (4.3.1) that there exists a sequence $j_{1}, j_{2}, \ldots, j_{N^{\prime}} \in I$ satisfying conditions $(\mathrm{A})$ and $\left(\mathrm{B}^{\prime}\right)$ for $\eta^{\prime}$. We set $\eta^{\prime \prime}:=e_{j_{N^{\prime}}}^{\max } e_{j_{N^{\prime}-1}}^{\max } \cdots e_{j_{1}}^{\max } \eta$. Then, repeated use of Lemma 4.2.3(2) shows that

$$
\eta^{\prime \prime}(t)=\left(e_{j_{N^{\prime}}}^{\max } e_{j_{N^{\prime}-1}}^{\max } \cdots e_{j_{1}}^{\max } \eta^{\prime}\right)(t)=\eta_{\mathrm{cl}(\lambda)}(t)=t \operatorname{cl}(\lambda) \quad \text { for } t \in\left[0, \sigma_{2}\right] .
$$

Hence the initial direction $\iota\left(\eta^{\prime \prime}\right)$, which equals $r_{j_{N^{\prime}}} r_{j_{N^{\prime}-1}} \cdots r_{j_{1}}(\operatorname{cl}(\lambda))$ by Lemma 4.2.3(2), must be equal to $\operatorname{cl}(\lambda)$. Therefore, if $\eta^{\prime \prime}=\left(\operatorname{cl}(\lambda), \mu_{2}^{\prime}, \ldots, \mu_{s^{\prime}}^{\prime} ; \sigma_{0}^{\prime}, \sigma_{1}^{\prime}, \sigma_{2}^{\prime}, \ldots, \sigma_{s^{\prime}}^{\prime}\right)$ is the reduced expression of $\eta^{\prime \prime}$, then we see from (4.3.2) that $\sigma_{1}^{\prime} \geqslant \sigma_{2}>\sigma_{1}$. Hence, by the induction hypothesis, there exists a sequence $j_{N^{\prime}+1}, j_{N^{\prime}+2}, \ldots, j_{N} \in I$ satisfying conditions $(\mathrm{A})$ and $\left(\mathrm{B}^{\prime}\right)$ for $\eta^{\prime \prime}$. Thus we obtain a sequence

$$
j_{1}, j_{2}, \ldots, j_{N^{\prime}}, j_{N^{\prime}+1}, j_{N^{\prime}+2}, \ldots, j_{N} \in I
$$

satisfying conditions $(\mathrm{A})$ and $\left(\mathrm{B}^{\prime}\right)$ for $\eta$ (note that $r_{j_{N^{\prime}}} r_{j_{N^{\prime}-1}} \cdots r_{j_{1}}(\operatorname{cl}(\lambda))=\iota\left(\eta^{\prime \prime}\right)=\operatorname{cl}(\lambda)$ ). This proves the assertion of the lemma in the general case.

Now, it remains to prove the assertion of the lemma in the special case (4.3.1). Since $\mathbb{B}(\lambda)_{\mathrm{cl}}$ is a finite set (see Remark 2.3.1), it follows that $\max \left\{\left\|\eta^{\prime}\right\| \mid \eta^{\prime} \in \mathbb{B}(\lambda)_{\mathrm{cl}}\right\}<\infty$, where $\|\eta\|$ is as defined in (2.3.1); recall that $\operatorname{wt}(\eta)$ is given by $\operatorname{wt}(\eta)=\eta(1) \in P_{\mathrm{cl}}$. We will show the assertion by descending induction on $\|\eta\|$. When $\|\eta\|=\max \left\{\left\|\eta^{\prime}\right\| \mid \eta^{\prime} \in \mathbb{B}(\lambda)_{\mathrm{cl}}\right\}$, it follows from Lemma 2.3.6 that $\eta$ is an extremal element. Since the initial direction $\iota(\eta)$ of $\eta$ is equal to $\operatorname{cl}(\lambda)$ by assumption, we deduce from Remark 2.4.3 that $\eta=\eta_{\mathrm{cl}(\lambda)}$ (hence there is nothing to prove).

Assume that $\|\eta\|<\max \left\{\left\|\eta^{\prime}\right\| \mid \eta^{\prime} \in \mathbb{B}(\lambda)_{\mathrm{cl}}\right\}$. Set $\Lambda:=w_{0} \lambda$, where $w_{0} \in \mathscr{W}^{W}$ is the longest element of $\dot{\circ}^{\circ}$. Then it is easy to check that $\Lambda\left(h_{j}\right) \in \mathbb{Z}_{<0}$ for all $j \in I_{0}$. Note that $\mu \in \operatorname{cl}(W \lambda)=\dot{\circ}^{\circ} \operatorname{cl}(\lambda)$ satisfies the condition that $\mu\left(h_{j}\right) \leqslant 0$ for all $j \in I_{0}$ if and only if $\mu=\operatorname{cl}(\Lambda)$ (see Remark 2.1.5).

We see from [AK97, Lemma 1.4] that there exists a sequence $j_{1}, j_{2}, \ldots, j_{N^{\prime}} \in I$ such that:

(a) $r_{j_{N^{\prime}}} r_{j_{N^{\prime}-1}} \cdots r_{j_{1}}(\operatorname{cl}(\lambda))=\operatorname{cl}(\Lambda)$;

(b) $\left(r_{j_{p}} r_{j_{p-1}} \cdots r_{j_{1}}(\operatorname{cl}(\lambda))\right)\left(h_{j_{p+1}}\right)<0$ for $p=0,1, \ldots, N^{\prime}-1$. 


\section{S. NAITO AND D. SAGAKI}

Set $\eta^{\prime}:=e_{j_{N^{\prime}}}^{\max } e_{j_{N^{\prime}-1}}^{\max } \cdots e_{j_{1}}^{\max } \eta \in \mathbb{B}(\lambda)_{\mathrm{cl}}$. Then it follows from Lemma 2.3.3 that

$$
\left\|\eta^{\prime}\right\| \geqslant\|\eta\| \text {. }
$$

Furthermore, from (b), we deduce by repeated application of Lemma 4.2.2 that

$$
e_{j_{N^{\prime}}}^{\max } e_{j_{N^{\prime}-1}}^{\max } \cdots e_{j_{1}}^{\max } \eta=\left(r_{j_{N^{\prime}}} r_{j_{N^{\prime}-1}} \cdots r_{j_{1}}(\operatorname{cl}(\lambda)), \mu^{\prime} ; 0, \sigma, 1\right) \quad \text { for some } \mu^{\prime} \in \operatorname{cl}(W \lambda),
$$

and hence from (a) that

$$
\begin{aligned}
\eta^{\prime} & =e_{j_{N^{\prime}}}^{\max } e_{j_{N^{\prime}-1} \max } \cdots e_{j_{1}}^{\max } \eta=\left(r_{j_{N^{\prime}}} r_{j_{N^{\prime}-1}} \cdots r_{j_{1}}(\operatorname{cl}(\lambda)), \mu^{\prime} ; 0, \sigma, 1\right) \\
& =\left(\operatorname{cl}(\Lambda), \mu^{\prime} ; 0, \sigma, 1\right) .
\end{aligned}
$$

Case 1: $\mu^{\prime}=\operatorname{cl}(\Lambda)$. In this case, we have $\eta^{\prime}=(\operatorname{cl}(\Lambda), \operatorname{cl}(\Lambda) ; 0, \sigma, 1)=\eta_{\mathrm{cl}(\Lambda)}$. Let $w_{0}=r_{j_{N^{\prime}+1}} r_{j_{N^{\prime}+2}} \ldots$ $r_{j_{N-1}} r_{j_{N}}$ be a reduced expression of $w_{0} \in \stackrel{\circ}{W}$; note that $j_{N^{\prime}+1}, j_{N^{\prime}+2}, \ldots, j_{N-1}, j_{N} \in I_{0}$. Then, since $\Lambda=w_{0} \lambda$, it follows that

(c) $r_{j_{N}} r_{j_{N-1}} \cdots r_{j_{N^{\prime}+1}}(\operatorname{cl}(\Lambda))=\operatorname{cl}(\lambda)$.

In addition, using [Kac90, Lemma 3.11b)], we obtain

(d) $\left(r_{j_{p}} r_{j_{p-1}} \cdots r_{j_{N^{\prime}+1}}(\operatorname{cl}(\Lambda))\right)\left(h_{j_{p+1}}\right)<0$ for all $p=N^{\prime}, N^{\prime}+1, \ldots, N-1$.

From (c) and (d), we see by repeated use of Lemma 4.2.2 that

$$
\begin{aligned}
& e_{j_{N}}^{\max } e_{j_{N-1}}^{\max } \cdots e_{j_{N^{\prime}+1}}^{\max } e_{j_{N^{\prime}}}^{\max } e_{j_{N^{\prime}-1}}^{\max } \cdots e_{j_{1}}^{\max } \eta=e_{j_{N}}^{\max } e_{j_{N-1}}^{\max } \cdots e_{j_{N^{\prime}+1}}^{\max } \eta^{\prime} \\
& \quad=e_{j_{N}}^{\max } e_{j_{N-1}}^{\max } \cdots e_{j_{N^{\prime}+1}}^{\max }(\operatorname{cl}(\Lambda), \operatorname{cl}(\Lambda) ; 0, \sigma, 1)=(\operatorname{cl}(\lambda), \operatorname{cl}(\lambda) ; 0, \sigma, 1)=\eta_{\operatorname{cl}(\lambda)} .
\end{aligned}
$$

Therefore, the sequence $j_{1}, j_{2}, \ldots, j_{N} \in I$ satisfies condition (A). Also, it follows from (a), (b), and (d) that $\left(r_{j_{p}} r_{j_{p-1}} \cdots r_{j_{1}}(\operatorname{cl}(\lambda))\right)\left(h_{j_{p+1}}\right)<0$ for all $p=0,1, \ldots, N-1$, and hence that the sequence $j_{1}, j_{2}, \ldots, j_{N} \in I$ satisfies condition $\left(\mathrm{B}^{\prime}\right)$.

Case 2: $\mu^{\prime} \neq \operatorname{cl}(\Lambda)$. In this case, we can take (and fix) $j_{N^{\prime}+1} \in I_{0}$ such that $\mu^{\prime}\left(h_{j_{N^{\prime}+1}}\right)>0$. Then, there exists a reduced expression of $w_{0} \in \stackrel{\circ}{W}$ of the form $w_{0}=r_{j_{N^{\prime}+1}} r_{j_{N^{\prime}+2}} \cdots r_{j_{N^{\prime \prime}}}$; note that $j_{N^{\prime}+1}, j_{N^{\prime}+2}, \ldots, j_{N^{\prime \prime}} \in I_{0}$. Since $\Lambda=w_{0} \lambda$, it follows that

(e) $r_{j_{N^{\prime \prime}}} r_{j_{N^{\prime \prime}-1}} \cdots r_{j_{N^{\prime}+1}}(\operatorname{cl}(\Lambda))=\operatorname{cl}(\lambda)$.

In addition, using [Kac90, Lemma 3.11b)], we obtain

(f) $\left(r_{j_{p}} r_{j_{p-1}} \cdots r_{j_{N^{\prime}+1}}(\operatorname{cl}(\Lambda))\right)\left(h_{j_{p+1}}\right)<0$ all for $p=N^{\prime}, N^{\prime}+1, \ldots, N^{\prime \prime}-1$.

If we set $\eta^{\prime \prime}:=e_{j_{N^{\prime \prime}}}^{\max } e_{j_{N^{\prime \prime}-1}}^{\max } \cdots e_{j_{N^{\prime}+1}}^{\max } \eta^{\prime}$, then $\left\|\eta^{\prime \prime}\right\|>\|\eta\|$. Indeed, since $(\operatorname{cl}(\Lambda))\left(h_{j_{N^{\prime}+1}}\right)<0$ by (f) with $p=N^{\prime}$, it follows from Lemma 2.2.11(1) that $e_{j_{N^{\prime}+1}} \eta^{\prime} \neq \mathbf{0}$. Also, since $\mu^{\prime}\left(h_{j_{N^{\prime}+1}}\right)>0$, it follows from Lemma 2.2.13 that $f_{j_{N^{\prime}+1}} \eta^{\prime} \neq \mathbf{0}$. Therefore, we obtain from Lemma 2.3.3

$$
\left\|e_{j_{N^{\prime}+1}}^{\max } \eta^{\prime}\right\|>\left\|\eta^{\prime}\right\|
$$

Hence we have

$$
\begin{aligned}
\left\|\eta^{\prime \prime}\right\| & \geqslant\left\|e_{j_{N^{\prime}+1}}^{\max } \eta^{\prime}\right\| \quad \text { by Lemma } 2.3 .3 \\
& >\left\|\eta^{\prime}\right\| \quad \text { by }(4.3 .4) \\
& \geqslant\|\eta\| \quad \text { by }(4.3 .3) .
\end{aligned}
$$

Furthermore, from (e) and (f), we see, by applying Lemma 4.2.2 successively, that

$$
\begin{aligned}
\eta^{\prime \prime} & =e_{j_{N^{\prime \prime}}}^{\max } e_{j_{N^{\prime \prime}-1}}^{\max } \cdots e_{j_{N^{\prime}+1}}^{\max } \eta^{\prime} \\
& =\left(r_{j_{N^{\prime \prime}}} r_{j_{N^{\prime \prime}-1}} \cdots r_{j_{N^{\prime}+1}}(\operatorname{cl}(\Lambda)), \mu^{\prime \prime} ; 0, \sigma, 1\right) \\
& =\left(\operatorname{cl}(\lambda), \mu^{\prime \prime} ; 0, \sigma, 1\right)
\end{aligned}
$$




\section{LS PATHS OF LEVEL-ZERO SHAPE AND 1DSUMS}

for some $\mu^{\prime \prime} \in \operatorname{cl}(W \lambda)$. Therefore, by the induction hypothesis, there exists a sequence $j_{N^{\prime \prime}+1}$, $j_{N^{\prime \prime}+2}, \ldots, j_{N} \in I$ satisfying conditions (A) and ( $\left.\mathrm{B}^{\prime}\right)$ for $\eta^{\prime \prime}$. It is easily checked by (a), (b), (e), (f), and the induction hypothesis that the sequence

$$
j_{1}, j_{2}, \ldots, j_{N^{\prime}}, j_{N^{\prime}+1}, j_{N^{\prime}+2}, \ldots, j_{N^{\prime \prime}}, j_{N^{\prime \prime}+1}, j_{N^{\prime \prime}+2}, \ldots, j_{N} \in I
$$

satisfies conditions $(\mathrm{A})$ and $\left(\mathrm{B}^{\prime}\right)$ for $\eta$. This proves the assertion in the special case (4.3.1), and hence completes the proof of the lemma.

If $\lambda \in \sum_{i \in I_{0}} \mathbb{Z}_{>0} \varpi_{i}$ is strictly $I_{0}$-dominant, then we see from Lemma 4.2.1(3) that condition $\left(\mathrm{B}^{\prime}\right)$ of Lemma 4.3.2 can be replaced by condition (B) of Proposition 4.3.1. Namely, we have the following result.

LEMma 4.3.3. Let $\lambda \in \sum_{i \in I_{0}} \mathbb{Z}_{>0} \varpi_{i}$ be a strictly $I_{0}$-dominant integral weight. Let $\eta$ be an element of $\mathbb{B}(\lambda)_{\mathrm{cl}}$ such that $\iota(\eta)=\operatorname{cl}(\lambda)$. Then, there exists a sequence $j_{1}, j_{2}, \ldots, j_{N} \in I$ satisfying conditions $(A)$ and $(B)$ of Proposition 4.3.1.

Finally, let us give a proof of Proposition 4.3.1.

Proof of Proposition 4.3.1. We set $\rho:=\sum_{i \in I_{0}} \varpi_{i} \in P_{+}^{0}$; note that $\rho \in P_{+}^{0}$ is strictly $I_{0}$-dominant, and hence so is $\lambda+\rho \in \sum_{i \in I} \mathbb{Z}_{>0} \varpi_{i}$. Since $e_{j} \eta=\mathbf{0}$ for all $j \in I_{0}$ by assumption and $e_{j} \eta_{\operatorname{cl}(\rho)}=\mathbf{0}$ for all $j \in I_{0}$ by the definition of the root operators $e_{j}$, we see from the tensor product rule for crystals that $\eta \otimes \eta_{\mathrm{cl}(\rho)} \in \mathbb{B}(\lambda)_{\mathrm{cl}} \otimes \mathbb{B}(\rho)_{\mathrm{cl}}$ also satisfies the condition that $e_{j}\left(\eta \otimes \eta_{\mathrm{cl}(\rho)}\right)=\mathbf{0}$ for all $j \in I_{0}$. Recall from Corollary 2.4.5(1) that there exists an isomorphism $\Psi_{\lambda, \rho}: \mathbb{B}(\lambda+\rho)_{\mathrm{cl}} \stackrel{\sim}{\longrightarrow} \mathbb{B}(\lambda)_{\mathrm{cl}} \otimes \mathbb{B}(\rho)_{\mathrm{cl}}$ of $P_{\mathrm{cl}}{ }^{-}$ crystals. Set $\eta^{\prime}:=\Psi_{\lambda, \rho}^{-1}\left(\eta \otimes \eta_{\mathrm{cl}(\rho)}\right) \in \mathbb{B}(\lambda+\rho)_{\mathrm{cl}}$. Then, clearly $e_{j} \eta^{\prime}=\mathbf{0}$ for all $j \in I_{0}$. Hence it follows from Lemma 2.2.11(1) (see also Remark 2.2.8) that the initial direction $\iota\left(\eta^{\prime}\right) \in \operatorname{cl}(W(\lambda+\rho))=$ $\stackrel{\circ}{W} \operatorname{cl}(\lambda+\rho)$ of $\eta^{\prime}$ is level-zero and $I_{0}$-dominant, and hence from Remark 2.1.5 that $\iota\left(\eta^{\prime}\right)=\operatorname{cl}(\lambda+\rho)$. Since $\lambda+\rho \in \sum_{i \in I} \mathbb{Z}_{>0} \varpi_{i}$ is strictly $I_{0}$-dominant, we know from Lemma 4.3.3 that there exists a sequence $j_{1}, j_{2}, \ldots, j_{N} \in I$ satisfying conditions (A) and (B) for $\eta^{\prime}$. Now, it remains to show that $e_{j_{N}}^{\max } e_{j_{N-1}}^{\max } \cdots e_{j_{1}}^{\max } \eta=\eta_{\mathrm{cl}(\lambda)}$, i.e. that the sequence $j_{1}, j_{2}, \ldots, j_{N} \in I$ also satisfies condition (A) for $\eta$. We have

$$
\begin{aligned}
& e_{j_{N}}^{\max } e_{j_{N-1}}^{\max } \cdots e_{j_{1}}^{\max }\left(\eta \otimes \eta_{\mathrm{cl}(\rho)}\right)=e_{j_{N}}^{\max } e_{j_{N-1}}^{\max } \cdots e_{j_{1}}^{\max }\left(\Psi_{\lambda, \rho}\left(\eta^{\prime}\right)\right) \\
& \quad=\Psi_{\lambda, \rho}\left(e_{j_{N}}^{\max } e_{j_{N-1}}^{\max } \cdots e_{j_{1}}^{\max } \eta^{\prime}\right)=\Psi_{\lambda, \rho}\left(\eta_{\mathrm{cl}(\lambda+\rho)}\right) \quad \text { by condition (A) for } \eta^{\prime} \\
& \quad=\eta_{\mathrm{cl}(\lambda)} \otimes \eta_{\mathrm{cl}(\rho)} \text { by }(2.4 .3) .
\end{aligned}
$$

Also, we see from Lemma 2.3.2(2) that

$$
e_{j_{N}}^{\max } e_{j_{N-1}}^{\max } \cdots e_{j_{1}}^{\max }\left(\eta \otimes \eta_{\mathrm{cl}(\rho)}\right)=\left(e_{j_{N}}^{\max } e_{j_{N-1}}^{\max } \cdots e_{j_{1}}^{\max } \eta\right) \otimes \eta^{\prime \prime} \quad \text { for some } \eta^{\prime \prime} \in \mathbb{B}(\rho)_{\mathrm{cl}} \text {. }
$$

Thus, we obtain $e_{j_{N}}^{\max } e_{j_{N-1}}^{\max } \cdots e_{j_{1}}^{\max } \eta=\eta_{\mathrm{cl}(\lambda)}$. This establishes Proposition 4.3.1.

\subsection{Proof of Theorem 4.1.2}

In this (and the next) subsection, the degree function Deg is defined on several different crystals $\mathbb{B}(\mu)_{\mathrm{cl}}, \mu \in P_{+}^{0}$. Accordingly, we write $\operatorname{Deg}_{\mu}(\eta)$ instead of $\operatorname{Deg}(\eta)$ for $\eta \in \mathbb{B}(\mu)_{\mathrm{cl}}$ with $\mu \in P_{+}^{0}$ to emphasize that $\eta$ is an element of $\mathbb{B}(\mu)_{\mathrm{cl}}$.

Let $\lambda, \lambda^{\prime} \in P_{+}^{0}$. For $\eta_{1} \otimes \eta_{2} \in \mathbb{B}(\lambda)_{\mathrm{cl}} \otimes \mathbb{B}\left(\lambda^{\prime}\right)_{\mathrm{cl}}$, we define (see Theorem 2.5.1)

$$
D_{\lambda, \lambda^{\prime}}\left(\eta_{1} \otimes \eta_{2}\right):=H_{\lambda, \lambda^{\prime}}\left(\eta_{1} \otimes \eta_{2}\right)+\operatorname{Deg}_{\lambda}\left(\eta_{1}\right)+\operatorname{Deg}_{\lambda^{\prime}}\left(\widetilde{\eta}_{2}\right)
$$

where $\widetilde{\eta}_{2} \in \mathbb{B}\left(\lambda^{\prime}\right)_{\mathrm{cl}}$ is defined by $\widetilde{\eta}_{2} \otimes \widetilde{\eta}_{1}=R_{\lambda, \lambda^{\prime}}\left(\eta_{1} \otimes \eta_{2}\right)$ (see Corollary 2.4.5(2)). From Theorem 2.5.1(H2) and Lemma 3.2.1(1), we deduce by use of (2.4.4) that

$$
D_{\lambda, \lambda^{\prime}}\left(\eta_{\operatorname{cl}(\lambda)} \otimes \eta_{\mathrm{cl}\left(\lambda^{\prime}\right)}\right)=H_{\lambda, \lambda^{\prime}}\left(\eta_{\operatorname{cl}(\lambda)} \otimes \eta_{\mathrm{cl}\left(\lambda^{\prime}\right)}\right)+\operatorname{Deg}_{\lambda}\left(\eta_{\operatorname{cl}(\lambda)}\right)+\operatorname{Deg}_{\lambda^{\prime}}\left(\eta_{\operatorname{cl}\left(\lambda^{\prime}\right)}\right)=0 .
$$




\section{S. NAito AND D. SAGAKI}

Lemma 4.4.1. Let $\lambda, \lambda^{\prime} \in P_{+}^{0}$, and let $\eta_{1} \otimes \eta_{2} \in \mathbb{B}(\lambda)_{\mathrm{cl}} \otimes \mathbb{B}\left(\lambda^{\prime}\right)_{\mathrm{cl}}$. The following hold:

(1) $D_{\lambda, \lambda^{\prime}}\left(e_{j}^{\max }\left(\eta_{1} \otimes \eta_{2}\right)\right)=D_{\lambda, \lambda^{\prime}}\left(\eta_{1} \otimes \eta_{2}\right)$ for all $j \in I_{0}$;

(2) if $\left(\iota\left(\eta_{1}\right)\right)\left(h_{0}\right) \leqslant 0$ and $\left(\iota\left(\widetilde{\eta}_{2}\right)\right)\left(h_{0}\right) \leqslant 0$, then

$$
D_{\lambda, \lambda^{\prime}}\left(e_{0}^{\max }\left(\eta_{1} \otimes \eta_{2}\right)\right)=D_{\lambda, \lambda^{\prime}}\left(\eta_{1} \otimes \eta_{2}\right)-\left(\iota\left(\eta_{1}\right)+\iota\left(\widetilde{\eta}_{2}\right)\right)\left(h_{0}\right)-\varepsilon_{0}\left(\eta_{1} \otimes \eta_{2}\right) .
$$

Proof. Let $j \in I$. We see from Lemma 2.3.2(2) that

$$
e_{j}^{\max }\left(\eta_{1} \otimes \eta_{2}\right)=e_{j}^{\max } \eta_{1} \otimes \eta_{2}^{\prime} \quad \text { for some } \eta_{2}^{\prime} \in \mathbb{B}\left(\lambda^{\prime}\right)_{\mathrm{cl}},
$$

and hence that

$$
\begin{aligned}
R_{\lambda, \lambda^{\prime}}\left(e_{j}^{\max }\left(\eta_{1} \otimes \eta_{2}\right)\right) & =e_{j}^{\max } R_{\lambda, \lambda^{\prime}}\left(\eta_{1} \otimes \eta_{2}\right)=e_{j}^{\max }\left(\widetilde{\eta}_{2} \otimes \widetilde{\eta}_{1}\right) \\
& =e_{j}^{\max } \widetilde{\eta}_{2} \otimes \eta_{1}^{\prime} \quad \text { for some } \eta_{1}^{\prime} \in \mathbb{B}(\lambda)_{\mathrm{cl}} .
\end{aligned}
$$

Therefore, from the definition (4.4.1) of $D_{\lambda, \lambda^{\prime}}$, we obtain

$$
D_{\lambda, \lambda^{\prime}}\left(e_{j}^{\max }\left(\eta_{1} \otimes \eta_{2}\right)\right)=H_{\lambda, \lambda^{\prime}}\left(e_{j}^{\max }\left(\eta_{1} \otimes \eta_{2}\right)\right)+\operatorname{Deg}_{\lambda}\left(e_{j}^{\max } \eta_{1}\right)+\operatorname{Deg}_{\lambda^{\prime}}\left(e_{j}^{\max } \widetilde{\eta}_{2}\right) .
$$

From (4.4.4), part (1) follows immediately by Theorem 2.5.1(H1) and Lemma 3.2.1(2). Let us prove part $(2)$. We give a proof only for the case $\varepsilon_{0}\left(\eta_{1}\right) \geqslant \varepsilon_{0}\left(\widetilde{\eta}_{2}\right)$; the proof for the case $\varepsilon_{0}\left(\eta_{1}\right)<\varepsilon_{0}\left(\widetilde{\eta}_{2}\right)$ is similar. By Lemma 3.2.2,

$$
\left\{\begin{array}{l}
\operatorname{Deg}_{\lambda}\left(e_{0}^{\max } \eta_{1}\right)=\operatorname{Deg}_{\lambda}\left(\eta_{1}\right)-\varepsilon_{0}\left(\eta_{1}\right)-\left(\iota\left(\eta_{1}\right)\right)\left(h_{0}\right) \\
\operatorname{Deg}_{\lambda^{\prime}}\left(e_{0}^{\max } \widetilde{\eta}_{2}\right)=\operatorname{Deg}_{\lambda^{\prime}}\left(\widetilde{\eta}_{2}\right)-\varepsilon_{0}\left(\widetilde{\eta}_{2}\right)-\left(\iota\left(\widetilde{\eta}_{2}\right)\right)\left(h_{0}\right) .
\end{array}\right.
$$

For simplicity of notation, we set $L:=\varepsilon_{0}\left(\eta_{1} \otimes \eta_{2}\right)$; note that $L=\varepsilon_{0}\left(\widetilde{\eta}_{2} \otimes \widetilde{\eta}_{1}\right)$ since $\widetilde{\eta}_{2} \otimes \widetilde{\eta}_{1}=$ $R_{\lambda, \lambda^{\prime}}\left(\eta_{1} \otimes \eta_{2}\right)$, and that $L \geqslant \varepsilon_{0}\left(\eta_{1}\right), \varepsilon_{0}\left(\widetilde{\eta}_{2}\right)$ by Lemma 2.3.2(1). It follows from Lemma 2.3.2(2) that, for $0 \leqslant l \leqslant L$,

$$
e_{0}^{l}\left(\eta_{1} \otimes \eta_{2}\right)= \begin{cases}\eta_{1} \otimes e_{0}^{l} \eta_{2} & \text { if } 0 \leqslant l \leqslant L-\varepsilon_{0}\left(\eta_{1}\right), \\ e_{0}^{l-L+\varepsilon_{0}\left(\eta_{1}\right)} \eta_{1} \otimes e_{0}^{L-\varepsilon_{0}\left(\eta_{1}\right)} \eta_{2} & \text { if } L-\varepsilon_{0}\left(\eta_{1}\right) \leqslant l \leqslant L,\end{cases}
$$

and

$$
e_{0}^{l}\left(\widetilde{\eta}_{2} \otimes \widetilde{\eta}_{1}\right)= \begin{cases}\widetilde{\eta}_{2} \otimes e_{0}^{l} \widetilde{\eta}_{1} & \text { if } 0 \leqslant l \leqslant L-\varepsilon_{0}\left(\widetilde{\eta}_{2}\right), \\ e_{0}^{l-L+\varepsilon_{0}\left(\widetilde{\eta}_{2}\right)} \widetilde{\eta}_{2} \otimes e_{0}^{L-\varepsilon_{0}\left(\widetilde{\eta}_{2}\right)} \widetilde{\eta}_{1} & \text { if } L-\varepsilon_{0}\left(\widetilde{\eta}_{2}\right) \leqslant l \leqslant L .\end{cases}
$$

Therefore, we deduce from Theorem 2.5.1(H1) that, for $0 \leqslant l^{\prime} \leqslant L-\varepsilon_{0}\left(\eta_{1}\right)$,

$$
H_{\lambda, \lambda^{\prime}}\left(e_{0}^{l^{\prime}}\left(\eta_{1} \otimes \eta_{2}\right)\right)=H_{\lambda, \lambda^{\prime}}\left(\eta_{1} \otimes \eta_{2}\right)-l^{\prime} .
$$

Similarly, we see from Theorem 2.5.1(H1) that, for $0 \leqslant l^{\prime} \leqslant \varepsilon_{0}\left(\eta_{1}\right)-\varepsilon_{0}\left(\widetilde{\eta}_{2}\right)$,

$$
\begin{aligned}
& H_{\lambda, \lambda^{\prime}}\left(e_{0}^{L-\varepsilon_{0}\left(\eta_{1}\right)+l^{\prime}}\left(\eta_{1} \otimes \eta_{2}\right)\right)=H_{\lambda, \lambda^{\prime}}\left(e_{0}^{L-\varepsilon_{0}\left(\eta_{1}\right)}\left(\eta_{1} \otimes \eta_{2}\right)\right) \\
& \quad=H_{\lambda, \lambda^{\prime}}\left(\eta_{1} \otimes \eta_{2}\right)-L+\varepsilon_{0}\left(\eta_{1}\right) \text { by }(4.4 .6) \text { with } l^{\prime}=L-\varepsilon_{0}\left(\eta_{1}\right),
\end{aligned}
$$

and that, for $0 \leqslant l^{\prime} \leqslant \varepsilon_{0}\left(\widetilde{\eta}_{2}\right)$,

$$
\begin{aligned}
& H_{\lambda, \lambda^{\prime}}\left(e_{0}^{L-\varepsilon_{0}\left(\widetilde{\eta}_{2}\right)+l^{\prime}}\left(\eta_{1} \otimes \eta_{2}\right)\right)=H_{\lambda, \lambda^{\prime}}\left(e_{0}^{L-\varepsilon_{0}\left(\widetilde{\eta}_{2}\right)}\left(\eta_{1} \otimes \eta_{2}\right)\right)+l^{\prime} \\
& \quad=H_{\lambda, \lambda^{\prime}}\left(\eta_{1} \otimes \eta_{2}\right)-L+\varepsilon_{0}\left(\eta_{1}\right)+l^{\prime} \quad \text { by }(4.4 .7) \text { with } l^{\prime}=\varepsilon_{0}\left(\eta_{1}\right)-\varepsilon_{0}\left(\widetilde{\eta}_{2}\right) .
\end{aligned}
$$

Finally, by taking $l^{\prime}=\varepsilon_{0}\left(\widetilde{\eta}_{2}\right)$ in (4.4.8), we conclude that

$$
\begin{aligned}
H_{\lambda, \lambda^{\prime}}\left(e_{0}^{\max }\left(\eta_{1} \otimes \eta_{2}\right)\right) & =H_{\lambda, \lambda^{\prime}}\left(e_{0}^{L}\left(\eta_{1} \otimes \eta_{2}\right)\right) \\
& =H_{\lambda, \lambda^{\prime}}\left(\eta_{1} \otimes \eta_{2}\right)-L+\varepsilon_{0}\left(\eta_{1}\right)+\varepsilon_{0}\left(\widetilde{\eta}_{2}\right) \\
& =H_{\lambda, \lambda^{\prime}}\left(\eta_{1} \otimes \eta_{2}\right)-\varepsilon_{0}\left(\eta_{1} \otimes \eta_{2}\right)+\varepsilon_{0}\left(\eta_{1}\right)+\varepsilon_{0}\left(\widetilde{\eta}_{2}\right) .
\end{aligned}
$$

By substituting (4.4.5) and (4.4.9) into (4.4.4), and then comparing the resulting equation with (4.4.1), we obtain the desired equation (4.4.3). This proves the lemma. 


\section{LS PATHS OF LEVEL-ZERO SHAPE AND 1DSUMS}

Proposition 4.4.2. Let $\lambda, \lambda^{\prime} \in P_{+}^{0}$, and let $\eta \in \mathbb{B}\left(\lambda+\lambda^{\prime}\right)_{\mathrm{cl}}$. Then,

$$
\operatorname{Deg}_{\lambda+\lambda^{\prime}}(\eta)=D_{\lambda, \lambda^{\prime}}\left(\Psi_{\lambda, \lambda^{\prime}}(\eta)\right)
$$

where $\Psi_{\lambda, \lambda^{\prime}}: \mathbb{B}\left(\lambda+\lambda^{\prime}\right)_{\mathrm{cl}} \stackrel{\sim}{\rightarrow} \mathbb{B}(\lambda)_{\mathrm{cl}} \otimes \mathbb{B}\left(\lambda^{\prime}\right)_{\mathrm{cl}}$ is the isomorphism of $P_{\mathrm{cl}}$-crystals in Corollary 2.4.5(1).

Proof. If $j \in I_{0}$, then it follows from Lemmas 3.2.1(2) and 4.4.1(1) that $\operatorname{Deg}_{\lambda+\lambda^{\prime}}\left(e_{j}^{\max } \eta\right)=$ $\operatorname{Deg}_{\lambda+\lambda^{\prime}}(\eta)$ and $D_{\lambda, \lambda^{\prime}}\left(\Psi_{\lambda, \lambda^{\prime}}\left(e_{j}^{\max } \eta\right)\right)=D_{\lambda, \lambda^{\prime}}\left(e_{j}^{\max }\left(\Psi_{\lambda, \lambda^{\prime}}(\eta)\right)\right)=D_{\lambda, \lambda^{\prime}}\left(\Psi_{\lambda, \lambda^{\prime}}(\eta)\right)$. Therefore, we may assume that $\eta \in \mathbb{B}\left(\lambda+\lambda^{\prime}\right)_{\mathrm{cl}}$ satisfies the condition that $e_{j} \eta=\mathbf{0}$ for all $j \in I_{0}$, since the $P_{\mathrm{cl}^{-}}$ crystal $\mathbb{B}\left(\lambda+\lambda^{\prime}\right)_{\mathrm{cl}}$ is regular. Then it follows from Lemma 2.2.11(1) (see also Remark 2.2.8) that $\iota(\eta) \in \operatorname{cl}(W \lambda)=\stackrel{\circ}{W} \operatorname{cl}(\lambda)$ is level-zero and $I_{0}$-dominant, and hence from Remark 2.1.5 that $\iota(\eta)=\operatorname{cl}\left(\lambda+\lambda^{\prime}\right)$. Set $\eta_{1} \otimes \eta_{2}:=\Psi_{\lambda, \lambda^{\prime}}(\eta) \in \mathbb{B}(\lambda)_{\mathrm{cl}} \otimes \mathbb{B}\left(\lambda^{\prime}\right)_{\mathrm{cl}}$, and $\widetilde{\eta}_{2} \otimes \widetilde{\eta}_{1}:=R_{\lambda, \lambda^{\prime}}\left(\eta_{1} \otimes \eta_{2}\right)$. Since $e_{j} \eta=\mathbf{0}$ for all $j \in I_{0}$, we have $e_{j}\left(\eta_{1} \otimes \eta_{2}\right)=\mathbf{0}$ and $e_{j}\left(\widetilde{\eta}_{2} \otimes \widetilde{\eta}_{1}\right)=\mathbf{0}$ for all $j \in I_{0}$, which implies that $e_{j} \eta_{1}=\mathbf{0}$ and $e_{j} \widetilde{\eta}_{2}=\mathbf{0}$ for all $j \in I_{0}$ by Lemma 2.3.2(1). Hence an argument similar to the above shows that $\iota\left(\eta_{1}\right)=\operatorname{cl}(\lambda)$ and $\iota\left(\widetilde{\eta}_{2}\right)=\operatorname{cl}\left(\lambda^{\prime}\right)$.

By Proposition 4.3.1, there exists a sequence $j_{1}, j_{2}, \ldots, j_{N} \in I$ satisfying conditions (A) and (B) for $\eta \in \mathbb{B}\left(\lambda+\lambda^{\prime}\right)_{\mathrm{cl}}$. Condition (B) implies that

$$
\left(w^{(p)}\left(\operatorname{cl}\left(\lambda+\lambda^{\prime}\right)\right)\right)\left(h_{j_{p+1}}\right) \leqslant 0 \quad \text { for } p=0,1, \ldots, N-1,
$$

by Lemma 4.2.1(2), where we set $w^{(p)}:=r_{j_{p}} r_{j_{p-1}} \cdots r_{j_{1}}$ for $0 \leqslant p \leqslant N$. Therefore, we see from Lemma 2.2.12 that

$$
\iota\left(E^{(p)} \eta\right)=w^{(p)}\left(\operatorname{cl}\left(\lambda+\lambda^{\prime}\right)\right) \text { for } p=0,1, \ldots, N,
$$

where we set $E^{(p)}:=e_{j_{p}}^{\max } e_{j_{p-1}}^{\max } \cdots e_{j_{1}}^{\max }$ for $0 \leqslant p \leqslant N$. Because $\iota\left(\eta_{1}\right)=\operatorname{cl}(\lambda)$ and $\iota\left(\widetilde{\eta}_{2}\right)=\operatorname{cl}\left(\lambda^{\prime}\right)$, an argument similar to the above shows that

$$
\begin{cases}\iota\left(E^{(p)} \eta_{1}\right)=w^{(p)}(\operatorname{cl}(\lambda)) & \text { for } p=0,1, \ldots, N, \\ \left(w^{(p)}(\operatorname{cl}(\lambda))\right)\left(h_{j_{p+1}}\right) \leqslant 0 & \text { for } p=0,1, \ldots, N-1,\end{cases}
$$

and

$$
\begin{cases}\iota\left(E^{(p)} \widetilde{\eta}_{2}\right)=w^{(p)}\left(\operatorname{cl}\left(\lambda^{\prime}\right)\right) & \text { for } p=0,1, \ldots, N \\ \left(w^{(p)}\left(\operatorname{cl}\left(\lambda^{\prime}\right)\right)\right)\left(h_{j_{p+1}}\right) \leqslant 0 & \text { for } p=0,1, \ldots, N-1 .\end{cases}
$$

Now we have

$$
\begin{aligned}
0 & =\operatorname{Deg}_{\lambda+\lambda^{\prime}}\left(\eta_{\mathrm{cl}\left(\lambda+\lambda^{\prime}\right)}\right) \quad \text { by Lemma } 3.2 .1(1) \\
& =\operatorname{Deg}_{\lambda+\lambda^{\prime}}\left(e_{j_{N}}^{\max } e_{j_{N-1}}^{\max } \cdots e_{j_{1}}^{\max } \eta\right) \quad \text { by condition (A) for } \eta \\
& =\operatorname{Deg}_{\lambda+\lambda^{\prime}}(\eta)-\sum_{1 \leqslant p \leqslant N ; j_{p}=0}\left\{\varepsilon_{0}\left(E^{(p-1)} \eta\right)+\left(w^{(p-1)}\left(\operatorname{cl}\left(\lambda+\lambda^{\prime}\right)\right)\right)\left(h_{j_{p}}\right)\right\}
\end{aligned}
$$

by Lemma 3.2.2 along with (4.4.11) and (4.4.12),

and hence

$$
\operatorname{Deg}_{\lambda+\lambda^{\prime}}(\eta)=\sum_{1 \leqslant p \leqslant N ; j_{p}=0}\left\{\varepsilon_{0}\left(E^{(p-1)} \eta\right)+\left(w^{(p-1)}\left(\operatorname{cl}\left(\lambda+\lambda^{\prime}\right)\right)\right)\left(h_{j_{p}}\right)\right\}
$$

Here, by Lemma 2.3.2(2),

$$
\begin{aligned}
E^{(p)}\left(\eta_{1} \otimes \eta_{2}\right) & =E^{(p)} \eta_{1} \otimes \eta_{2}^{\prime} \quad \text { for some } \eta_{2}^{\prime} \in \mathbb{B}\left(\lambda^{\prime}\right)_{\mathrm{cl}}, \\
R_{\lambda, \lambda^{\prime}}\left(E^{(p)}\left(\eta_{1} \otimes \eta_{2}\right)\right) & =E^{(p)}\left(R_{\lambda, \lambda^{\prime}}\left(\eta_{1} \otimes \eta_{2}\right)\right)=E^{(p)}\left(\widetilde{\eta}_{2} \otimes \widetilde{\eta}_{1}\right) \\
& =E^{(p)} \widetilde{\eta}_{2} \otimes \eta_{1}^{\prime} \quad \text { for some } \eta_{1}^{\prime} \in \mathbb{B}(\lambda)_{\mathrm{cl}} .
\end{aligned}
$$




\section{S. NAITO AND D. SAGAKI}

Therefore, we see that

$$
\begin{aligned}
0 & =D_{\lambda, \lambda^{\prime}}\left(\eta_{\mathrm{cl}(\lambda)} \otimes \eta_{\mathrm{cl}\left(\lambda^{\prime}\right)}\right) \quad \text { by }(4.4 .2) \\
& =D_{\lambda, \lambda^{\prime}}\left(e_{j_{N}}^{\max } e_{j_{N-1}}^{\max } \cdots e_{j_{1}}^{\max }\left(\eta_{1} \otimes \eta_{2}\right)\right) \quad \text { by condition }(\mathrm{A}) \text { for } \eta \text { and }(2.4 .3) \\
& =D_{\lambda, \lambda^{\prime}}\left(\eta_{1} \otimes \eta_{2}\right)-\sum_{1 \leqslant p \leqslant N ; j_{p}=0}\left\{\varepsilon_{0}\left(E^{(p-1)}\left(\eta_{1} \otimes \eta_{2}\right)\right)+\left(w^{(p-1)}\left(\operatorname{cl}\left(\lambda+\lambda^{\prime}\right)\right)\right)\left(h_{j_{p}}\right)\right\}
\end{aligned}
$$

by Lemma 4.4.1 along with (4.4.13) and (4.4.14).

Also, since $\Psi_{\lambda, \lambda^{\prime}}\left(E^{(p)} \eta\right)=E^{(p)} \Psi_{\lambda, \lambda^{\prime}}(\eta)=E^{(p)}\left(\eta_{1} \otimes \eta_{2}\right)$, it follows that $\varepsilon_{0}\left(E^{(p)} \eta\right)=\varepsilon_{0}\left(E^{(p)}\left(\eta_{1} \otimes \eta_{2}\right)\right)$ for all $p=0,1, \ldots, N$. Thus we obtain

$$
D_{\lambda, \lambda^{\prime}}\left(\eta_{1} \otimes \eta_{2}\right)=\sum_{1 \leqslant p \leqslant N ; j_{p}=0}\left\{\varepsilon_{0}\left(E^{(p-1)} \eta\right)+\left(w^{(p-1)}\left(\operatorname{cl}\left(\lambda+\lambda^{\prime}\right)\right)\right)\left(h_{j_{p}}\right)\right\} .
$$

Equation (4.4.10) follows immediately from (4.4.15) and (4.4.16). This completes the proof of the proposition.

Now we are ready to prove Theorem 4.1.2.

Proof of Theorem 4.1.2. We proceed by induction on the length $n$ of the sequence $\mathbf{i}=\left(i_{1}, i_{2}, \ldots, i_{n}\right)$. When $n=1$, the assertion obviously holds. Assume that $n>1$, and set $\mathbf{i}^{\prime}:=\left(i_{1}, i_{2}, \ldots, i_{n-1}\right)$, $\lambda^{\prime}:=\lambda-\varpi_{i_{n}} \in P_{+}^{0}$. Recall from Theorem 2.4.1 and Corollary 2.4.5(1) that there exist isomorphisms

$$
\Psi_{\mathbf{i}^{\prime}}: \mathbb{B}\left(\lambda^{\prime}\right)_{\mathrm{cl}} \stackrel{\sim}{\longrightarrow} \mathbb{B}_{\mathbf{i}^{\prime}}=\mathbb{B}\left(\varpi_{i_{1}}\right)_{\mathrm{cl}} \otimes \mathbb{B}\left(\varpi_{i_{2}}\right)_{\mathrm{cl}} \otimes \cdots \otimes \mathbb{B}\left(\varpi_{i_{n-1}}\right)_{\mathrm{cl}}
$$

and

$$
\Psi_{\lambda^{\prime}, \varpi_{i_{n}}}: \mathbb{B}(\lambda)_{\mathrm{cl}} \stackrel{\sim}{\longrightarrow} \mathbb{B}\left(\lambda^{\prime}\right)_{\mathrm{cl}} \otimes \mathbb{B}\left(\varpi_{i_{n}}\right)_{\mathrm{cl}}
$$

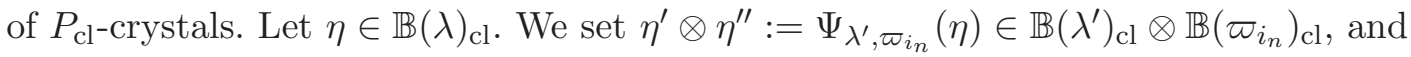

$$
\eta_{1} \otimes \eta_{2} \otimes \cdots \otimes \eta_{n}:=\Psi_{\mathbf{i}}(\eta) \in \mathbb{B}_{\mathbf{i}}=\mathbb{B}\left(\varpi_{i_{1}}\right)_{\mathrm{cl}} \otimes \mathbb{B}\left(\varpi_{i_{2}}\right)_{\mathrm{cl}} \otimes \cdots \otimes \mathbb{B}\left(\varpi_{i_{n}}\right)_{\mathrm{cl}} .
$$

Note that both of $\Psi_{\mathbf{i}}$ and $\left(\Psi_{\mathbf{i}^{\prime}} \otimes \mathrm{id}\right) \circ \Psi_{\lambda^{\prime}, \varpi_{i_{n}}}$ are isomorphisms of $P_{\mathrm{cl}}$-crystals from $\mathbb{B}(\lambda)_{\mathrm{cl}}$ to $\mathbb{B}_{\mathbf{i}}$. Since the $P_{\mathrm{cl}}$-crystals $\mathbb{B}(\lambda)_{\mathrm{cl}}$ and $\mathbb{B}_{\mathbf{i}}$ are both simple, it follows from Lemma 2.3.9(3) that $\Psi_{\mathbf{i}}=$ $\left(\Psi_{\mathbf{i}^{\prime}} \otimes \mathrm{id}\right) \circ \Psi_{\lambda^{\prime}, \varpi_{i_{n}}}$, and hence that

$$
\eta^{\prime \prime}=\eta_{n} \quad \text { and } \quad \Psi_{\mathbf{i}^{\prime}}\left(\eta^{\prime}\right)=\eta_{1} \otimes \eta_{2} \otimes \cdots \otimes \eta_{n-1} .
$$

We see from Proposition 4.4.2 that

$$
\operatorname{Deg}_{\lambda}(\eta)=D_{\lambda^{\prime}, \varpi_{i_{n}}}\left(\eta^{\prime} \otimes \eta^{\prime \prime}\right)=H_{\lambda^{\prime}, \varpi_{i_{n}}}\left(\eta^{\prime} \otimes \eta^{\prime \prime}\right)+\operatorname{Deg}_{\lambda^{\prime}}\left(\eta^{\prime}\right)+\operatorname{Deg}_{\varpi_{i_{n}}}\left(\widetilde{\eta}^{\prime \prime}\right),
$$

where we set $\widetilde{\eta}^{\prime \prime} \otimes \widetilde{\eta}^{\prime}:=R_{\lambda^{\prime}, \varpi_{i_{n}}}\left(\eta^{\prime} \otimes \eta^{\prime \prime}\right)$. Here we remark that the element $\eta_{n}^{(1)} \in \mathbb{B}\left(\varpi_{i_{n}}\right)_{\mathrm{cl}}$ (for the definition of $\eta_{n}^{(1)}$, see $\left.\S 4.1\right)$ is the first factor of the image of $\eta_{1} \otimes \eta_{2} \otimes \cdots \otimes \eta_{n} \in \mathbb{B}_{\mathbf{i}}$ under the (unique) isomorphism (id $\left.\otimes \Psi_{\mathbf{i}^{\prime}}\right) \circ R_{\lambda^{\prime}, \varpi_{i_{n}}} \circ \Psi_{\lambda^{\prime}, \varpi_{i_{n}}} \circ \Psi_{\mathbf{i}}^{-1}$ of $P_{\mathrm{cl}}$-crystals, which is obtained as follows:

$$
\begin{gathered}
\mathbb{B}\left(\varpi_{i_{1}}\right)_{\mathrm{cl}} \otimes \mathbb{B}\left(\varpi_{i_{2}}\right)_{\mathrm{cl}} \otimes \cdots \otimes \mathbb{B}\left(\varpi_{i_{n}}\right)_{\mathrm{cl}}=\mathbb{B}_{\mathbf{i}} \stackrel{\Psi_{\mathrm{i}}^{-1}}{\sim} \mathbb{B}(\lambda)_{\mathrm{cl}} \stackrel{\Psi_{\lambda^{\prime}, \varpi_{i_{n}}}}{\sim} \mathbb{B}\left(\lambda^{\prime}\right)_{\mathrm{cl}} \otimes \mathbb{B}\left(\varpi_{i_{n}}\right)_{\mathrm{cl}} \\
\stackrel{R_{\lambda^{\prime}, \varpi_{i n}}}{\sim} \mathbb{B}\left(\varpi_{i_{n}}\right)_{\mathrm{cl}} \otimes \mathbb{B}\left(\lambda^{\prime}\right)_{\mathrm{cl}} \stackrel{\mathrm{id} \otimes \Psi_{\mathrm{i}^{\prime}}}{\sim} \mathbb{B}\left(\varpi_{i_{n}}\right)_{\mathrm{cl}} \otimes \mathbb{B}\left(\varpi_{i_{1}}\right)_{\mathrm{cl}} \otimes \cdots \otimes \mathbb{B}\left(\varpi_{i_{n-1}}\right)_{\mathrm{cl}} .
\end{gathered}
$$

Also, it is easy to check that this first factor is identical to $\widetilde{\eta}^{\prime \prime} \in \mathbb{B}\left(\varpi_{i_{n}}\right)_{\mathrm{cl}}$. Thus we have $\eta_{n}^{(1)}=\widetilde{\eta}^{\prime \prime}$. Furthermore, using (4.4.17), we see from [Oka07, Lemma 5.2] that

$$
H_{\lambda^{\prime}, \varpi_{i_{n}}}\left(\eta^{\prime} \otimes \eta^{\prime \prime}\right)=\sum_{1 \leqslant k \leqslant n-1} H_{\varpi_{i_{k}}, \varpi_{i_{n}}}\left(\eta_{k} \otimes \eta_{n}^{(k+1)}\right) .
$$




\section{LS PATHS OF LEVEL-ZERO SHAPE AND 1DSUMS}

Now, the induction hypothesis along with (4.4.17) implies that

$$
\operatorname{Deg}_{\lambda^{\prime}}\left(\eta^{\prime}\right)=\sum_{1 \leqslant k<l \leqslant n-1} H_{\varpi_{i_{k}}, \varpi_{i_{l}}}\left(\eta_{k} \otimes \eta_{l}^{(k+1)}\right)+\sum_{k=1}^{n-1} \operatorname{Deg}_{\varpi_{i_{k}}}\left(\eta_{k}^{(1)}\right) .
$$

By substituting (4.4.19) and (4.4.20) into (4.4.18), and using the fact that $\eta_{n}^{(1)}=\widetilde{\eta}^{\prime \prime}$, we obtain

$$
\begin{aligned}
\operatorname{Deg}_{\lambda}(\eta)= & H_{\lambda^{\prime}, \varpi_{i_{n}}}\left(\eta^{\prime} \otimes \eta^{\prime \prime}\right)+\operatorname{Deg}_{\lambda^{\prime}}\left(\eta^{\prime}\right)+\operatorname{Deg}_{\varpi_{i_{n}}}\left(\widetilde{\eta}^{\prime \prime}\right) \\
= & \sum_{1 \leqslant k \leqslant n-1} H_{\varpi_{i_{k}}, \varpi_{i_{n}}}\left(\eta_{k} \otimes \eta_{n}^{(k+1)}\right) \\
& +\sum_{1 \leqslant k<l \leqslant n-1} H_{\varpi_{i_{k}}, \varpi_{i_{l}}}\left(\eta_{k} \otimes \eta_{l}^{(k+1)}\right)+\sum_{k=1}^{n-1} \operatorname{Deg}_{\varpi_{i_{k}}}\left(\eta_{k}^{(1)}\right)+\operatorname{Deg}_{\varpi_{i_{n}}}\left(\eta_{n}^{(1)}\right) \\
= & \sum_{1 \leqslant k<l \leqslant n} H_{\varpi_{i_{k}}, \varpi_{i_{l}}}\left(\eta_{k} \otimes \eta_{l}^{(k+1)}\right)+\sum_{k=1}^{n} \operatorname{Deg}_{\varpi_{i_{k}}}\left(\eta_{k}^{(1)}\right) .
\end{aligned}
$$

This completes the proof of (4.1.4), thereby establishing Theorem 4.1.2.

\subsection{Proof of Theorem 4.1.3}

In this subsection, we continue to write $\operatorname{Deg}_{\mu}(\eta)$ instead of $\operatorname{Deg}(\eta)$ in the case that $\eta$ is an element of $\mathbb{B}(\mu)_{\mathrm{cl}}$ for $\mu \in P_{+}^{0}$.

Fix an arbitrary $i \in I_{0}$. Recall from Corollary 2.4.5(1) that there exists an isomorphism $\Psi_{\varpi_{i}, \varpi_{i}}$ : $\mathbb{B}\left(2 \varpi_{i}\right)_{\mathrm{cl}} \stackrel{\sim}{\longrightarrow} \mathbb{B}\left(\varpi_{i}\right)_{\mathrm{cl}} \otimes \mathbb{B}\left(\varpi_{i}\right)_{\mathrm{cl}}$ of $P_{\mathrm{cl}}$-crystals. The next lemma follows from the proof of [NS06, Proposition 3.4.4].

Lemma 4.5.1. Let $\eta_{1} \otimes \eta_{2} \in \mathbb{B}\left(\varpi_{i}\right)_{\mathrm{cl}} \otimes \mathbb{B}\left(\varpi_{i}\right)_{\mathrm{cl}}$. Then, the preimage $\Psi_{\varpi_{i}, \varpi_{i}}^{-1}\left(\eta_{1} \otimes \eta_{2}\right) \in \mathbb{B}\left(2 \varpi_{i}\right)_{\mathrm{cl}}$ of $\eta_{1} \otimes \eta_{2} \in \mathbb{B}\left(\varpi_{i}\right)_{\mathrm{cl}} \otimes \mathbb{B}\left(\varpi_{i}\right)_{\mathrm{cl}}$ under the isomorphism $\Psi_{\varpi_{i}, \varpi_{i}}$ is identical to the concatenation $\eta_{1} * \eta_{2}$ of $\eta_{1}$ and $\eta_{2}$ defined by

$$
\left(\eta_{1} * \eta_{2}\right)(t)= \begin{cases}\eta_{1}(2 t) & \text { if } 0 \leqslant t \leqslant 1 / 2, \\ \eta_{1}(1)+\eta_{2}(2 t-1) & \text { if } 1 / 2 \leqslant t \leqslant 1 .\end{cases}
$$

In addition, from [NS06, Proposition 3.2.2], we have the next result.

Lemma 4.5.2. The set $\mathbb{B}\left(2 \varpi_{i}\right)$ is identical to the set of all concatenations $\pi_{1} * \pi_{2}$ of LS paths $\pi_{1}, \pi_{2} \in \mathbb{B}\left(\varpi_{i}\right)$ such that $\kappa\left(\pi_{1}\right) \geqslant \iota\left(\pi_{2}\right)$. Here, the concatenation $\pi_{1} * \pi_{2}$ is defined by the same formula as (4.5.1), with $\eta_{1}$ and $\eta_{2}$ replaced by $\pi_{1}$ and $\pi_{2}$, respectively.

Lemma 4.5.3. Let $\eta \in \mathbb{B}\left(2 \varpi_{i}\right)_{\mathrm{cl}}$, and set $\eta_{1} \otimes \eta_{2}:=\Psi_{\varpi_{i}, \varpi_{i}}(\eta) \in \mathbb{B}\left(\varpi_{i}\right)_{\mathrm{cl}} \otimes \mathbb{B}\left(\varpi_{i}\right)_{\mathrm{cl}}$. Then,

$$
\operatorname{Deg}_{2 \varpi_{i}}(\eta)=H_{\varpi_{i}, \varpi_{i}}\left(\eta_{1} \otimes \eta_{2}\right)+2 \operatorname{Deg}_{\varpi_{i}}\left(\eta_{1}\right) \text {. }
$$

Proof. Applying Theorem 4.1.2 to the case in which $\mathbf{i}=(i, i)$ and hence $\lambda=2 \varpi_{i}$, we obtain

$$
\begin{aligned}
\operatorname{Deg}_{2 \varpi_{i}}(\eta) & =H_{\varpi_{i}, \varpi_{i}}\left(\eta_{1} \otimes \eta_{2}^{(2)}\right)+\operatorname{Deg}_{\varpi_{i}}\left(\eta_{1}^{(1)}\right)+\operatorname{Deg}_{\varpi_{i}}\left(\eta_{2}^{(1)}\right) \\
& =H_{\varpi_{i}, \varpi_{i}}\left(\eta_{1} \otimes \eta_{2}\right)+\operatorname{Deg}_{\varpi_{i}}\left(\eta_{1}\right)+\operatorname{Deg}_{\varpi_{i}}\left(\eta_{2}^{(1)}\right),
\end{aligned}
$$

since $\eta_{1}^{(1)}=\eta_{1}$ and $\eta_{2}^{(2)}=\eta_{2}$ by definition. Also, from Lemma 2.3.9(3) and the definition of $\eta_{2}^{(1)}$, we deduce that $\eta_{2}^{(1)}=\eta_{1}$, since the $P_{\mathrm{cl}}$-crystal $\mathbb{B}\left(\varpi_{i}\right)_{\mathrm{cl}} \otimes \mathbb{B}\left(\varpi_{i}\right)_{\mathrm{cl}}$ is simple. Thus we obtain (4.5.2). This proves the lemma.

Let us fix an (arbitrary) element $\eta^{b} \in \mathbb{B}\left(\varpi_{i}\right)_{\mathrm{cl}}$ such that $f_{j} \eta^{b}=\mathbf{0}$ for all $j \in I_{0}$. Then it follows from (2.2.10) that $f_{j} \pi_{\eta^{b}}=\mathbf{0}$ for all $j \in I_{0}$. Therefore, by Lemma 2.2.13, the final direction 


\section{S. Naito And D. SAGaki}

$\nu^{b}:=\kappa\left(\pi_{\eta^{b}}\right) \in W \varpi_{i}$ of $\pi_{\eta^{b}}$ satisfies the condition that $\nu^{b}\left(h_{j}\right) \leqslant 0$ for all $j \in I_{0}$. Hence $\operatorname{cl}\left(\nu^{b}\right)=$ $w_{0}\left(\operatorname{cl}\left(\varpi_{i}\right)\right)$ by Remark 2.1.5. From this, we deduce (using Lemma 2.1.2) that $\nu^{b} \in W \varpi_{i}$ can be written as $\nu^{b}=w_{0} \varpi_{i}+k^{b} d_{\varpi_{i}} \delta$ for some $k^{b} \in \mathbb{Z}$.

Lemma 4.5.4. Let $\eta \in \mathbb{B}\left(\varpi_{i}\right)_{\mathrm{cl}}$, and set $\eta^{\prime}:=\Psi_{\varpi_{i}, \varpi_{i}}^{-1}\left(\eta^{b} \otimes \eta\right) \in \mathbb{B}\left(2 \varpi_{i}\right)_{\mathrm{cl}}$. The following hold:

(1) the path $\pi_{\eta^{\mathrm{b}}} *\left(\pi_{\eta}+\pi_{k^{\mathrm{b}} d_{\varpi_{i}} \delta}\right)$ lies in $\mathrm{cl}^{-1}\left(\eta^{\prime}\right) \cap \mathbb{B}\left(2 \varpi_{i}\right)$;

(2) $\pi_{\eta^{\prime}}=\pi_{\eta^{b}} *\left(\pi_{\eta}+\pi_{k^{b} d_{\varpi_{i}} \delta}\right)$.

Proof. (1) First, note that $\eta^{\prime}$ is identical to the concatenation $\eta^{b} * \eta$ by Lemma 4.5.1. Also, by the definition of concatenations (see (4.5.1)),

$$
\operatorname{cl}\left(\pi_{\eta^{\mathrm{b}}} *\left(\pi_{\eta}+\pi_{k^{\mathrm{b}} d_{\varpi_{i}} \delta}\right)\right)=\operatorname{cl}\left(\pi_{\eta^{\mathrm{b}}}\right) * \operatorname{cl}\left(\pi_{\eta}+\pi_{k^{\mathrm{b}} d_{\varpi_{i}} \delta}\right) .
$$

Since $\operatorname{cl}\left(\pi_{\eta^{\mathrm{b}}}\right)=\eta^{\mathrm{b}}$ and $\operatorname{cl}\left(\pi_{\eta}+\pi_{k^{\mathrm{b}}{\varpi_{\varpi}}_{i} \delta}\right)=\operatorname{cl}\left(\pi_{\eta}\right)=\eta$, we have

$$
\operatorname{cl}\left(\pi_{\eta^{\mathrm{b}}} *\left(\pi_{\eta}+\pi_{k^{\mathrm{b}} d_{\varpi_{i}} \delta}\right)\right)=\operatorname{cl}\left(\pi_{\eta^{\mathrm{b}}}\right) * \operatorname{cl}\left(\pi_{\eta}+\pi_{k^{\mathrm{b}} d_{\varpi_{i}} \delta}\right)=\eta^{\mathrm{b}} * \eta=\eta^{\prime},
$$

and hence $\pi_{\eta^{\mathrm{b}}} *\left(\pi_{\eta}+\pi_{k^{\mathrm{b}} d_{\varpi_{i}} \delta}\right) \in \mathrm{cl}^{-1}\left(\eta^{\prime}\right)$.

Next, we show that $\pi_{\eta^{b}} *\left(\pi_{\eta}+\pi_{k^{b} d_{\varpi_{i}} \delta}\right) \in \mathbb{B}\left(2 \varpi_{i}\right)$. Note that $\pi_{\eta}+\pi_{k^{b} d_{\varpi_{i}} \delta} \in \mathbb{B}\left(\varpi_{i}\right)$ by Lemma 2.2.6. Therefore, by Lemma 4.5.2, it suffices to show that $\iota\left(\pi_{\eta}+\pi_{k^{b} d_{\varpi_{i}} \delta}\right) \leqslant \nu^{b}=\kappa\left(\pi_{\eta^{b}}\right)$. Let us write $\iota\left(\pi_{\eta}\right) \in \varpi_{i}-\stackrel{\circ}{Q}_{+}$as $\iota\left(\pi_{\eta}\right)=w \varpi_{i}$ for some $w \in \stackrel{\circ}{W}$ (see Lemma 2.1.2). Since $w_{0} \in \stackrel{\circ}{W}$ is greater than or equal to $w \in \stackrel{\circ}{W}$ with respect to the usual Bruhat ordering on the (finite) Weyl group $\stackrel{\circ}{W}$ of $\mathfrak{g}_{I_{0}}$, it follows (see [Lit95, Remark 4.2]) that $w_{0} \varpi_{i} \geqslant w \varpi_{i}$. From this, using the definition of the ordering $\geqslant$ on $W \varpi_{i}$, we see that

$$
\nu^{b}=w_{0} \varpi_{i}+k^{b} d_{\varpi_{i}} \delta \geqslant w \varpi_{i}+k^{b} d_{\varpi_{i}} \delta=\iota\left(\pi_{\eta}\right)+k^{b} d_{\varpi_{i}} \delta .
$$

Hence, by Remark 2.2.4, we obtain

$$
\iota\left(\pi_{\eta}+\pi_{k^{b} d_{\varpi_{i}} \delta}\right)=\iota\left(\pi_{\eta}\right)+\iota\left(\pi_{k^{b} d_{\varpi_{i}} \delta}\right)=\iota\left(\pi_{\eta}\right)+k^{b} d_{\varpi_{i}} \delta \leqslant w_{0} \varpi_{i}+k^{b} d_{\varpi_{i}} \delta=\nu^{b} .
$$

This proves part (1).

(2) First, by the definitions of $\operatorname{Deg}_{\varpi_{i}}\left(\eta^{b}\right)$ and $\operatorname{Deg}_{\varpi_{i}}(\eta)$, we can write $\pi_{\eta^{b}}(1) \in P$ and $\pi_{\eta}(1) \in P$ as $\pi_{\eta^{b}}(1)=\varpi_{i}-a_{0}^{-1} \beta^{b}-a_{0}^{-1} \operatorname{Deg}_{\varpi_{i}}\left(\eta^{b}\right)$ and $\pi_{\eta}(1)=\varpi_{i}-a_{0}^{-1} \beta-a_{0}^{-1} \operatorname{Deg}_{\varpi_{i}}(\eta)$ for some $\beta^{b}, \beta \in \stackrel{Q}{+}_{+}$, respectively. Hence we have

$$
\begin{aligned}
& \left(\pi_{\eta^{b}} *\left(\pi_{\eta}+\pi_{k^{b} d_{\varpi_{i}} \delta}\right)\right)(1)=\pi_{\eta^{b}}(1)+\left(\pi_{\eta}+\pi_{k^{b} d_{\varpi_{i}} \delta}\right)(1)=\pi_{\eta^{b}}(1)+\pi_{\eta}(1)+\pi_{k^{b} d_{\varpi_{i}} \delta}(1) \\
& \quad=\left\{\varpi_{i}-a_{0}^{-1} \beta^{b}-a_{0}^{-1} \operatorname{Deg}_{\varpi_{i}}\left(\eta^{b}\right)\right\}+\left\{\varpi_{i}-a_{0}^{-1} \beta-a_{0}^{-1} \operatorname{Deg}_{\varpi_{i}}(\eta)\right\}+k^{b} d_{\varpi_{i}} \delta \\
& \quad=2 \varpi_{i}-a_{0}^{-1}\left(\beta^{b}+\beta\right)-a_{0}^{-1}\left(\operatorname{Deg}_{\varpi_{i}}\left(\eta^{b}\right)+\operatorname{Deg}_{\varpi_{i}}(\eta)-a_{0} k^{b} d_{\varpi_{i}}\right) \delta .
\end{aligned}
$$

Now, in view of Proposition 3.1.4(1), it suffices to show the following:

$$
\begin{gathered}
\iota\left(\pi_{\eta^{b}} *\left(\pi_{\eta}+\pi_{k^{b} d_{\varpi_{i}} \delta}\right)\right) \in 2 \varpi_{i}-\stackrel{Q}{+}_{+}, \\
\operatorname{Deg}_{2 \varpi_{i}}\left(\eta^{\prime}\right) \leqslant \operatorname{Deg}_{\varpi_{i}}\left(\eta^{b}\right)+\operatorname{Deg}_{\varpi_{i}}(\eta)-a_{0} k^{b} d_{\varpi_{i}} .
\end{gathered}
$$

As for (4.5.5), we easily deduce from the definition of concatenations that $\iota\left(\pi_{\eta^{\mathrm{b}}} *\left(\pi_{\eta}+\pi_{k^{\mathrm{b}} d_{\varpi_{i}} \delta}\right)\right)=$ $2 \iota\left(\pi_{\eta^{\mathrm{b}}}\right)$, where the right-hand side lies in $2 \varpi_{i}-\stackrel{\circ}{Q}_{+}$since $\iota\left(\pi_{\eta^{\mathrm{b}}}\right) \in \varpi_{i}-\stackrel{\circ}{Q}_{+}$by definition.

Let us show (4.5.6). Set $\pi_{1}(t):=\pi_{\eta^{\prime}}(t / 2)$ and $\pi_{2}(t):=\pi_{\eta^{\prime}}((t+1) / 2)-\pi_{\eta^{\prime}}(1 / 2)$ for $t \in[0,1]$. Then it is obvious that $\pi_{\eta^{\prime}}=\pi_{1} * \pi_{2}$. Also, we see from the proof of [NS06, Proposition 3.2.2] that $\pi_{1}, \pi_{2} \in \mathbb{B}\left(\varpi_{i}\right)$. Furthermore, since $\eta^{b} * \eta=\eta^{\prime}=\operatorname{cl}\left(\pi_{\eta^{\prime}}\right)=\operatorname{cl}\left(\pi_{1}\right) * \operatorname{cl}\left(\pi_{2}\right)$, we deduce that $\operatorname{cl}\left(\pi_{1}\right)=\eta^{b}$ and $\operatorname{cl}\left(\pi_{2}\right)=\eta$. 


\section{LS PATHS OF LEVEL-ZERO SHAPE AND 1DSUMS}

Since $\iota\left(\pi_{\eta^{\prime}}\right) \in 2 \varpi_{i}-\stackrel{\circ}{Q}_{+}$, and since $\iota\left(\pi_{1}\right)=\frac{1}{2} \iota\left(\pi_{\eta^{\prime}}\right)$ by the definition of $\pi_{1} \in \mathbb{B}\left(\varpi_{i}\right)$, it follows from Lemma 2.1.2 that $\iota\left(\pi_{1}\right) \in W \varpi_{i}$ lies in $\varpi_{i}-Q_{+}$. If we write $\pi_{1}(1) \in P$ in the form $\pi_{1}(1)=$ $\varpi_{i}-a_{0}^{-1} \beta_{1}+a_{0}^{-1} K_{1} \delta$ with $\beta_{1} \in \stackrel{Q}{Q}_{+}$and $K_{1} \in \mathbb{Z}_{\geqslant 0}$ (see Lemma 3.1.1), then by Proposition 3.1.4(1) applied to $\operatorname{cl}\left(\pi_{1}\right)=\eta^{\mathrm{b}} \in \mathbb{B}\left(\varpi_{i}\right)_{\mathrm{cl}}$, we have

$$
-K_{1} \leqslant \operatorname{Deg}_{\varpi_{i}}\left(\eta^{b}\right) .
$$

Also, if we write $\kappa\left(\pi_{1}\right) \in W \varpi_{i}$ in the form $\varpi_{i}-\beta_{1}^{\prime}+k_{1}^{\prime} d_{\varpi_{i}} \delta$ with $\beta_{1}^{\prime} \in \stackrel{\circ}{Q}_{+}$and $k_{1}^{\prime} \in \mathbb{Z}$, then by Proposition 3.1.4(2) applied to $\operatorname{cl}\left(\pi_{1}\right)=\eta^{b} \in \mathbb{B}\left(\varpi_{i}\right)_{\mathrm{cl}}$, we have

$$
k_{1}^{\prime} \geqslant k^{b} \text {, }
$$

where we recall from the discussion preceding this lemma that $\nu^{b}=\kappa\left(\pi_{\eta^{b}}\right) \in W \varpi_{i}$ equals $w_{0} \varpi_{i}+$ $k^{b} d_{\varpi_{i}} \delta$.

Since $\pi_{\eta^{\prime}}=\pi_{1} * \pi_{2} \in \mathbb{B}\left(2 \varpi_{i}\right)$, it follows from Lemma 4.5.2 that $\iota\left(\pi_{2}\right) \leqslant \kappa\left(\pi_{1}\right)=\varpi_{i}-\beta_{1}^{\prime}+k_{1}^{\prime} d_{\varpi_{i}} \delta$. Hence, by Lemma 2.1.2 and Remark 2.1.3, $\iota\left(\pi_{2}\right) \in W \varpi_{i}$ is of the form $\iota\left(\pi_{2}\right)=\varpi_{i}-\beta_{2}^{\prime}+k_{2}^{\prime} d_{\varpi_{i}} \delta$, with $\beta_{2}^{\prime} \in \stackrel{\circ}{Q}_{+}$and $k_{2}^{\prime} \in \mathbb{Z}$ such that

$$
k_{2}^{\prime} \geqslant k_{1}^{\prime} \text {. }
$$

If we set $\pi_{2}^{\prime}:=\pi_{2}-\pi_{k_{2}^{\prime} d_{\varpi_{i}} \delta}$, then it follows from Lemma 2.2.6 that $\pi_{2}^{\prime} \in \mathbb{B}\left(\varpi_{i}\right)$. Also, we have $\operatorname{cl}\left(\pi_{2}^{\prime}\right)=\operatorname{cl}\left(\pi_{2}\right)=\eta$, and by Remark 2.2.4, $\iota\left(\pi_{2}^{\prime}\right)=\iota\left(\pi_{2}\right)-k_{2}^{\prime} d_{\varpi_{i}} \delta=\varpi_{i}-\beta_{2}^{\prime} \in \varpi_{i}-\dot{Q}_{+}$. Hence, if we write $\pi_{2}^{\prime}(1) \in P$ in the form $\pi_{2}^{\prime}(1)=\varpi_{i}-a_{0}^{-1} \beta_{2}+a_{0}^{-1} K_{2} \delta$ with $\beta_{2} \in \stackrel{\circ}{Q}_{+}$and $K_{2} \in \mathbb{Z}_{\geqslant 0}$ (see Lemma 3.1.1), then by Proposition 3.1.4(1) applied to $\operatorname{cl}\left(\pi_{2}^{\prime}\right)=\eta \in \mathbb{B}\left(\varpi_{i}\right)_{\mathrm{cl}}$, we have

$$
-K_{2} \leqslant \operatorname{Deg}_{\varpi_{i}}(\eta) \text {. }
$$

From the above, we see that

$$
\begin{aligned}
\pi_{\eta^{\prime}}(1) & =\left(\pi_{1} * \pi_{2}\right)(1)=\pi_{1}(1)+\pi_{2}(1)=\pi_{1}(1)+\pi_{2}^{\prime}(1)+k_{2}^{\prime} d_{\varpi_{i}} \delta \\
& =\left(\varpi_{i}-a_{0}^{-1} \beta_{1}+a_{0}^{-1} K_{1} \delta\right)+\left(\varpi_{i}-a_{0}^{-1} \beta_{2}+a_{0}^{-1} K_{2} \delta\right)+k_{2}^{\prime} d_{\varpi_{i}} \delta \\
& =2 \varpi_{i}-a_{0}^{-1}\left(\beta_{1}+\beta_{2}\right)+a_{0}^{-1}\left(K_{1}+K_{2}+a_{0} k_{2}^{\prime} d_{\varpi_{i}}\right) \delta,
\end{aligned}
$$

and hence that $\operatorname{Deg}_{2 \varpi_{i}}\left(\eta^{\prime}\right)=-K_{1}-K_{2}-a_{0} k_{2}^{\prime} d_{\varpi_{i}}$. By (4.5.7), (4.5.10), and the inequalities (4.5.8) and (4.5.9), we obtain

$$
\operatorname{Deg}_{2 \varpi_{i}}\left(\eta^{\prime}\right)=-K_{1}-K_{2}-a_{0} k_{2}^{\prime} d_{\varpi_{i}} \leqslant \operatorname{Deg}_{\varpi_{i}}\left(\eta^{b}\right)+\operatorname{Deg}_{\varpi_{i}}(\eta)-a_{0} k^{b} d_{\varpi_{i}},
$$

which is the desired inequality (4.5.6). This completes the proof of part (2).

Now we are ready to prove Theorem 4.1.3.

Proof of Theorem 4.1.3. Let $\eta \in \mathbb{B}\left(\varpi_{i}\right)_{\mathrm{cl}}$, and set $\eta^{\prime}:=\Psi_{\varpi_{i}, \varpi_{i}}^{-1}\left(\eta^{b} \otimes \eta\right) \in \mathbb{B}\left(2 \varpi_{i}\right)_{\mathrm{cl}}$. It follows from Lemma 4.5.3 that

$$
\operatorname{Deg}_{2 \varpi_{i}}\left(\eta^{\prime}\right)=H_{\varpi_{i}, \varpi_{i}}\left(\eta^{b} \otimes \eta\right)+2 \operatorname{Deg}_{\varpi_{i}}\left(\eta^{b}\right) .
$$

Also, we deduce from Lemma 4.5.4(2) and the definition of $\operatorname{Deg}\left(\eta^{\prime}\right)$, by using (4.5.4), that

$$
\operatorname{Deg}_{2 \varpi_{i}}\left(\eta^{\prime}\right)=\operatorname{Deg}_{\varpi_{i}}\left(\eta^{b}\right)+\operatorname{Deg}_{\varpi_{i}}(\eta)-a_{0} k^{b} d_{\varpi_{i}} .
$$

Hence, by combining (4.5.11) and (4.5.12), we obtain

$$
H_{\varpi_{i}, \varpi_{i}}\left(\eta^{b} \otimes \eta\right)=\operatorname{Deg}_{\varpi_{i}}(\eta)-\operatorname{Deg}_{\varpi_{i}}\left(\eta^{b}\right)-a_{0} k^{b} d_{\varpi_{i}} .
$$

In particular, by taking $\eta=\eta_{\mathrm{cl}\left(\varpi_{i}\right)} \in \mathbb{B}\left(\varpi_{i}\right)_{\mathrm{cl}}$ in (4.5.13), we obtain

$$
H_{\varpi_{i}, \varpi_{i}}\left(\eta^{b} \otimes \eta_{\mathrm{cl}\left(\varpi_{i}\right)}\right)=\operatorname{Deg}_{\varpi_{i}}\left(\eta_{\mathrm{cl}\left(\varpi_{i}\right)}\right)-\operatorname{Deg}_{\varpi_{i}}\left(\eta^{b}\right)-a_{0} k^{b} d_{\varpi_{i}} .
$$




\section{S. NAITO AND D. SAGAKI}

Since $\operatorname{Deg}_{\varpi_{i}}\left(\eta_{\mathrm{cl}\left(\varpi_{i}\right)}\right)=0$ by Lemma 3.2.1(1), it follows that

$$
H_{\varpi_{i}, \varpi_{i}}\left(\eta^{b} \otimes \eta_{\mathrm{cl}\left(\varpi_{i}\right)}\right)=-\operatorname{Deg}_{\varpi_{i}}\left(\eta^{b}\right)-a_{0} k^{b} d_{\varpi_{i}} .
$$

Equation (4.1.5) follows immediately from (4.5.13) and (4.5.14). This establishes Theorem 4.1.3.

\section{Relation to one-dimensional sums}

In this section, as an application of Theorem 4.1.1, we provide an expression for classically restricted one-dimensional sums (1dsums for short) and, in the case of type $A_{\ell-1}^{(1)}$, an expression for KostkaFoulkes polynomials in terms of LS paths.

\subsection{Expression for one-dimensional sums in terms of LS paths}

Here we recall from [HKOTY99, Conjecture 2.1] and [HKOTT02, Conjecture 2.1] a conjectural family of finite-dimensional irreducible $U_{q}^{\prime}(\mathfrak{g})$-modules $W_{s}^{(i)}$ having crystal bases $\mathcal{B}^{i, s}$ indexed by $i \in I_{0}$ and $s \in \mathbb{Z}_{\geqslant 1}$ for which the decomposition into irreducible $U_{q}\left(\mathfrak{g}_{I_{0}}\right)$-modules is prescribed by the $\mathrm{KR}$ conjecture, presented in [KR90]; the $U_{q}^{\prime}(\mathfrak{g})$-module $W_{s}^{(i)}$ is called a KR module, and the crystal $\mathcal{B}^{i, s}$ is called a KR crystal. Through enough evidence (see Introduction and references therein), it is confirmed that the level-zero fundamental representation $W\left(\varpi_{i}\right)$ is indeed the (conjectural) KR module $W_{s}^{(i)}$ with $s=1$, and hence the crystal basis $\mathcal{B}\left(W\left(\varpi_{i}\right)\right)$ of $W\left(\varpi_{i}\right)$ is the KR crystal $\mathcal{B}^{i, 1}$ for every $i \in I_{0}$. Thus, the (conjectural) $\mathrm{KR}$ crystal $\mathcal{B}^{i, s}$ with $s=1$ is obtained as the crystal $\mathbb{B}\left(\varpi_{i}\right)_{\mathrm{cl}}$ for all $i \in I_{0}$.

In [HKOTY99] and [HKOTT02], a $q$-analogue of the KR conjecture (called the $X=M$ conjecture) was proposed by introducing a classically restricted one-dimensional sum (1dsum for short) $X$ associated to a tensor product of $\mathrm{KR}$ crystals $\mathcal{B}^{i, s}, i \in I_{0}, s \in \mathbb{Z}_{\geqslant 1}$, and a fermionic formula $M$, which is a specific sum of products of $q$-binomial coefficients. (We will not pursue the fermionic formula $M$ in this paper.) Following the definition in [HKOTY99, §3] and [HKOTT02, §3.3] of classically restricted 1 dsums, we define a classically restricted 1 dsum associated to a tensor product of simple crystals $\mathbb{B}\left(\varpi_{i}\right)_{\mathrm{cl}} \simeq \mathcal{B}\left(W\left(\varpi_{i}\right)\right), i \in I_{0}$, with $\mathbb{B}\left(\varpi_{i}\right)_{\mathrm{cl}}$ in place of $\mathcal{B}^{i, 1}$ for $i \in I_{0}$, as follows. Let $\mathbf{i}=\left(i_{1}, i_{2}, \ldots, i_{n}\right)$ be a sequence of elements of $I_{0}$, and $\mathbb{B}_{\mathbf{i}}=\mathbb{B}\left(\varpi_{i_{1}}\right)_{\mathrm{cl}} \otimes \mathbb{B}\left(\varpi_{i_{2}}\right)_{\mathrm{cl}} \otimes \cdots \otimes \mathbb{B}\left(\varpi_{i_{n}}\right)_{\mathrm{cl}}$. Then, for an element $\mu \in \operatorname{cl}\left(P_{+}^{0}\right)=\sum_{i \in I_{0}} \mathbb{Z}_{\geqslant 0} \operatorname{cl}\left(\varpi_{i}\right)$, the classically restricted 1 dsum $X\left(\mathbb{B}_{\mathbf{i}}, \mu ; q\right)$ is defined by

$$
X\left(\mathbb{B}_{\mathbf{i}}, \mu ; q\right)=\sum_{\substack{b \in \mathbb{B}_{\mathbf{i}} \\ e_{j}=\mathbf{0}\left(j \in I_{0}\right) \\ \text { wt } b=\mu}} q^{D_{\mathbf{i}}(b)},
$$

where $D_{\mathbf{i}}: \mathbb{B}_{\mathbf{i}} \rightarrow \mathbb{Z}$ is as defined in (4.1.1). Because the isomorphism $\Psi_{\mathbf{i}}: \mathbb{B}(\lambda)_{\mathrm{cl}} \stackrel{\sim}{\longrightarrow} \mathbb{B}_{\mathbf{i}}$ of $P_{\mathrm{cl}}$-crystals is compatible with the root operators $e_{j}, j \in I_{0}$, on $\mathbb{B}(\lambda)_{\mathrm{cl}}$ and the root operators $e_{j}, j \in I_{0}$, on $\mathbb{B}_{\mathbf{i}}$, we obtain the following corollary of Theorem 4.1.1.

Corollary 5.1.1. Let $\mathbf{i}=\left(i_{1}, i_{2}, \ldots, i_{n}\right)$ be a sequence of elements of $I_{0}$, and set $\lambda:=\sum_{k=1}^{n} \varpi_{i_{k}} \in$ $P_{+}^{0}$. For every $\mu \in \operatorname{cl}\left(P_{+}^{0}\right)=\sum_{i \in I_{0}} \mathbb{Z}_{\geqslant 0} \operatorname{cl}\left(\varpi_{i}\right)$, the following equation holds:

$$
\sum_{\substack{\eta \in \mathbb{B}(\lambda)_{\mathrm{cl}} \\ e_{j} \eta=\mathbf{0}\left(j \in I_{0}\right) \\ \eta(1)=\mu}} q^{\operatorname{Deg}(\eta)}=q^{-D_{\mathbf{i}}^{\text {ext }} X\left(\mathbb{B}_{\mathbf{i}}, \mu ; q\right) .}
$$

Remark 5.1.2 (see also [HKOTT02, Proposition 3.9]). Let $\lambda \in P_{+}^{0}$, and let $\mu \in \operatorname{cl}\left(P_{+}^{0}\right)$. We see

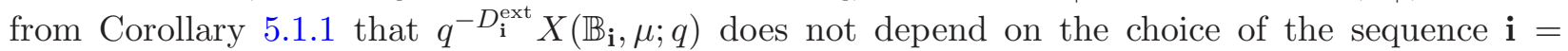
$\left(i_{1}, i_{2}, \ldots, i_{n}\right)$ of elements of $I_{0}$ such that $\lambda=\sum_{k=1}^{n} \varpi_{i_{k}}$. 


\section{LS PATHS OF LEVEL-ZERO SHAPE AND 1DSUMS}

\subsection{Expression for Kostka-Foulkes polynomials in terms of LS paths}

Let $i \in I_{0}$. Recall the element $\eta_{\widetilde{\varpi}_{i}} \in \mathbb{B}\left(\varpi_{i}\right)_{\mathrm{cl}}$ introduced in $\S 4.1$, where $\widetilde{\varpi}_{i}=w_{0} \operatorname{cl}\left(\varpi_{i}\right) \in P_{\mathrm{cl}}$. We note that if the crystal $\operatorname{res}_{I_{0}} \mathbb{B}\left(\varpi_{i}\right)_{\mathrm{cl}}$ is connected (see $\S 2.3$ for the definition of $\operatorname{res}_{I_{0}} \mathbb{B}\left(\varpi_{i}\right)_{\mathrm{cl}}$ ), then the element $\eta_{\widetilde{\varpi}_{i}}$ is the unique element of $\mathbb{B}\left(\varpi_{i}\right)_{\mathrm{cl}}$ such that $f_{j} \eta_{\widetilde{\varpi}_{i}}=\mathbf{0}$ for all $j \in I_{0}$, since the $P_{\mathrm{cl}}$-crystal $\mathbb{B}\left(\varpi_{i}\right)_{\mathrm{cl}}$ is regular.

Lemma 5.2.1. Let $i \in I_{0}$, and assume that the crystal $\operatorname{res}_{I_{0}} \mathbb{B}\left(\varpi_{i}\right)_{\mathrm{cl}}$ is connected. Then, the equation $H_{\varpi_{i}, \varpi_{i}}$ $\left(\eta_{\widetilde{\varpi}_{i}} \otimes \eta\right)=0$ holds for all $\eta \in \mathbb{B}\left(\varpi_{i}\right)_{\mathrm{cl}}$.

Remark 5.2.2. Since the $P_{\mathrm{cl}}$-crystal $\mathbb{B}\left(\varpi_{i}\right)_{\mathrm{cl}}$ is regular, we see that $\operatorname{res}_{I_{0}} \mathbb{B}\left(\varpi_{i}\right)_{\mathrm{cl}}$ is connected if and only if $W\left(\varpi_{i}\right)$ is irreducible when regarded as a $U_{q}^{\prime}(\mathfrak{g})_{I_{0}}$-module by restriction. Therefore, we deduce from $\left[\operatorname{Kas} 05\right.$, Lemma 4.3(ii)] that, if $a_{i}^{\vee}=1$, then $\operatorname{res}_{I_{0}} \mathbb{B}\left(\varpi_{i}\right)_{\mathrm{cl}}$ is connected.

Proof of Lemma 5.2.1. Since $\operatorname{res}_{I_{0}} \mathbb{B}\left(\varpi_{i}\right)_{\mathrm{cl}}$ is connected by the assumption of the lemma, there exists a monomial $X$ in the root operators $f_{j}$ for $j \in I_{0}$ such that $X \eta=\eta_{\widetilde{\varpi}_{i}}$. Since $f_{j} \eta_{\widetilde{\varpi}_{i}}=\mathbf{0}$ for all $j \in I_{0}$, it follows from the tensor product rule for crystals that

$$
X\left(\eta_{\widetilde{\varpi}_{i}} \otimes \eta\right)=\eta_{\widetilde{\varpi}_{i}} \otimes X \eta=\eta_{\widetilde{\varpi}_{i}} \otimes \eta_{\widetilde{\varpi}_{i}} .
$$

Furthermore, we see from the proof of [AK97, Lemma 1.6(1)] that

$$
S_{w_{0}} X\left(\eta_{\widetilde{\varpi}_{i}} \otimes \eta\right)=S_{w_{0}}\left(\eta_{\widetilde{\varpi}_{i}} \otimes \eta_{\widetilde{w}_{i}}\right)=\left(S_{w_{0}} \eta_{\widetilde{\varpi}_{i}}\right) \otimes\left(S_{w_{0}} \eta_{\widetilde{w}_{i}}\right),
$$

and from Remark 2.4.3 that $S_{w_{0}} \eta_{\widetilde{\varpi}_{i}}=\eta_{\mathrm{cl}\left(\varpi_{i}\right)}$. Since $w_{0} \in \stackrel{\circ}{W}$, it follows from the definition of $S_{w_{0}}$ (see (2.3.2)) that there exists a monomial $X^{\prime}$ in the root operators $e_{j}, f_{j}$ for $j \in I_{0}$ such that $S_{w_{0}} X\left(\eta_{\widetilde{\varpi}_{i}} \otimes \eta\right)=X^{\prime} X\left(\eta_{\widetilde{\varpi}_{i}} \otimes \eta\right)$. Therefore, we obtain

$$
\begin{aligned}
0 & =H_{\varpi_{i}, \varpi_{i}}\left(\eta_{\operatorname{cl}\left(\varpi_{i}\right)} \otimes \eta_{\operatorname{cl}\left(\varpi_{i}\right)}\right) \quad \text { by Theorem } 2.5 .1 \\
& =H_{\varpi_{i}, \varpi_{i}}\left(\left(S_{w_{0}} \eta_{\widetilde{\varpi}_{i}}\right) \otimes\left(S_{w_{0}} \eta_{\widetilde{\varpi}_{i}}\right)\right) \\
& =H_{\varpi_{i}, \varpi_{i}}\left(S_{w_{0}} X\left(\eta_{\widetilde{\varpi}_{i}} \otimes \eta\right)\right) \\
& =H_{\varpi_{i}, \varpi_{i}}\left(X^{\prime} X\left(\eta_{\widetilde{\varpi}_{i}} \otimes \eta\right)\right) \\
& =H_{\varpi_{i}, \varpi_{i}}\left(\eta_{\widetilde{\varpi}_{i}} \otimes \eta\right) \quad \text { by Theorem } 2.5 .1(\mathrm{H} 1) .
\end{aligned}
$$

This proves the lemma.

Remark 5.2.3. Let $\mathbf{i}=\left(i_{1}, i_{2}, \ldots, i_{n}\right)$ be a sequence of elements of $I_{0}$ such that $a_{i_{k}}^{\vee}=1$ for all $1 \leqslant k \leqslant n$. Then we know from Remark 5.2.2 that $\operatorname{res}_{I_{0}} \mathbb{B}\left(\varpi_{i_{k}}\right)_{\mathrm{cl}}$ is connected for all $1 \leqslant k \leqslant n$. Therefore, we see from Lemma 5.2.1 and the definitions of $D_{\mathbf{i}}$ and $D_{\mathbf{i}}^{\text {ext }}$ that, for every

$$
\eta_{1} \otimes \eta_{2} \otimes \cdots \otimes \eta_{n} \in \mathbb{B}_{\mathbf{i}}=\mathbb{B}\left(\varpi_{i_{1}}\right)_{\mathrm{cl}} \otimes \mathbb{B}\left(\varpi_{i_{2}}\right)_{\mathrm{cl}} \otimes \cdots \otimes \mathbb{B}\left(\varpi_{i_{n}}\right)_{\mathrm{cl}},
$$

we have

$$
D_{\mathbf{i}}\left(\eta_{1} \otimes \eta_{2} \otimes \cdots \otimes \eta_{n}\right)=\sum_{1 \leqslant k<l \leqslant n} H_{\varpi_{i_{k}}, \varpi_{i_{l}}}\left(\eta_{k} \otimes \eta_{l}^{(k+1)}\right) \quad \text { and } \quad D_{\mathbf{i}}^{\text {ext }}=0
$$

Now we restrict our attention to the case in which $\mathfrak{g}$ is of type $A_{\ell-1}^{(1)}$. Because $a_{i}^{\vee}=1$ for all $i \in I_{0}$ in the case of type $A_{\ell-1}^{(1)}$, we know from Remark 5.2.2 that $\operatorname{res}_{I_{0}} \mathbb{B}\left(\varpi_{i}\right)_{\mathrm{cl}}$ is connected for all $i \in I_{0}$. In fact, we can check by direct calculation that every $\varpi_{i}$ is 'minuscule', i.e. that $\varpi_{i}\left(\beta^{\vee}\right) \in\{0, \pm 1\}$ for all $\beta \in \stackrel{\circ}{\Delta}:=\stackrel{\circ}{W} \Pi_{I_{0}} \subset \Delta^{\text {re }}$, where $\Pi_{I_{0}}=\left\{\alpha_{j}\right\}_{j \in I_{0}}$, and hence that $\varpi_{i}\left(\xi^{\vee}\right) \in\{0, \pm 1\}$ for all $\xi \in \Delta_{+}^{\text {re }}$. Using this fact, we deduce from the definition of LS paths that

$$
\mathbb{B}\left(\varpi_{i}\right)_{\mathrm{cl}}=\left\{\eta_{\mu} \mid \mu \in \operatorname{cl}\left(W \varpi_{i}\right)=\stackrel{\circ}{W} \operatorname{cl}\left(\varpi_{i}\right)\right\}
$$




\section{S. NAito AND D. SAGAKI}

for every $i \in I_{0}$. Also, it follows from the definition of the root operators $e_{j}$ (respectively, $f_{j}$ ), $j \in I$, that, for $\mu \in \stackrel{\circ}{W} \operatorname{cl}\left(\varpi_{i}\right)$ and $j \in I$, the following hold:

(1) $e_{j} \eta_{\mu} \neq \mathbf{0}$ (respectively, $f_{j} \eta_{\mu} \neq \mathbf{0}$ ) if and only if $\mu\left(h_{j}\right)=-1$ (respectively, $=1$ );

(2) if $e_{j} \eta_{\mu} \neq \mathbf{0}$ (respectively, $f_{j} \eta_{\mu} \neq \mathbf{0}$ ), then $e_{j} \eta_{\mu}$ (respectively, $\left.f_{j} \eta_{\mu}\right)=\eta_{r_{j} \mu}$, with $r_{j}(\mu)=\mu+\operatorname{cl}\left(\alpha_{j}\right)$ (respectively, $=\mu-\operatorname{cl}\left(\alpha_{j}\right)$ ).

Here we recall from [NY97, $\S \S 2.1$ and 2.4] the $P_{\mathrm{cl}}$-crystals $B_{\mathrm{cl}\left(\varpi_{i}\right)}$ (adapted to our notation) for $i \in I_{0}=\{1,2, \ldots, \ell-1\}$. Let $i \in I_{0}=\{1,2, \ldots, \ell-1\}$. Then, the crystal $B_{\mathrm{cl}\left(\varpi_{i}\right)}$ consists of all Young diagrams of shape $\left(1^{\ell}\right)$ having $i$ dots (in all) with at most one dot in each box. The weight $\mathrm{wt}(b) \in P_{\mathrm{cl}}$ of the element $b \in B_{\mathrm{cl}\left(\varpi_{i}\right)}$ having dots exactly in the $j_{1}$ th box, $j_{2}$ th box $, \ldots, j_{i}$ th box with $1 \leqslant j_{1}<j_{2}<\cdots<j_{i} \leqslant \ell$, is given by $\gamma_{j_{1}}+\gamma_{j_{2}}+\cdots+\gamma_{j_{i}} \in P_{\mathrm{cl}}$, where we set $\gamma_{j}:=\operatorname{cl}\left(\varpi_{j}\right)-\operatorname{cl}\left(\varpi_{j-1}\right)$ for $1 \leqslant j \leqslant \ell$, with $\varpi_{0}=\varpi_{\ell}=0 \in P$.

Now, for each $i \in I_{0}=\{1,2, \ldots, \ell-1\}$, we define a map $\Phi_{i}: B_{\operatorname{cl}\left(\varpi_{i}\right)} \rightarrow \mathbb{B}\left(\varpi_{i}\right)_{\mathrm{cl}}$ by $\Phi_{i}(b)=\eta_{\mathrm{wt}(b)}$ for $b \in B_{\operatorname{cl}\left(\varpi_{i}\right)}$, which is easily seen to be a (well-defined) bijection that preserves weights, since the subgroup $\stackrel{\circ}{W} \subset W$ is isomorphic to the symmetric group $S_{\ell}$ permuting the $\gamma_{j}, 1 \leqslant j \leqslant \ell$, and since $\operatorname{cl}\left(\varpi_{i}\right)=\gamma_{1}+\gamma_{2}+\cdots+\gamma_{i}$. Actually, the map $\Phi_{i}: B_{\mathrm{cl}\left(\varpi_{i}\right)} \rightarrow \mathbb{B}\left(\varpi_{i}\right)_{\mathrm{cl}}$ is an isomorphism of $P_{\mathrm{cl}}$-crystals. Indeed, from the definition in [NY97, § 2.4] of the Kashiwara operators $e_{j}$ (respectively, $\left.f_{j}\right), j \in I$, on $B_{\mathrm{cl}\left(\varpi_{i}\right)}$, we see that, for $b \in B_{\mathrm{cl}\left(\varpi_{i}\right)}$ and $j \in I, e_{j} b \neq \mathbf{0}$ (respectively, $f_{j} b \neq \mathbf{0}$ ) holds if and only if $(\operatorname{wt}(b))\left(h_{j}\right)=-1$ (respectively, $\left.=1\right)$, since $\gamma_{j}=\operatorname{cl}\left(\varpi_{j}\right)-\operatorname{cl}\left(\varpi_{j-1}\right)=\operatorname{cl}\left(\Lambda_{j}\right)-\operatorname{cl}\left(\Lambda_{j-1}\right)$ for $2 \leqslant j \leqslant \ell-1$, and $\gamma_{1}=\operatorname{cl}\left(\varpi_{1}\right)=\operatorname{cl}\left(\Lambda_{1}\right)-\operatorname{cl}\left(\Lambda_{0}\right), \gamma_{\ell}=-\operatorname{cl}\left(\varpi_{\ell-1}\right)=-\operatorname{cl}\left(\Lambda_{\ell-1}\right)+\operatorname{cl}\left(\Lambda_{0}\right)$. Also, if $e_{j} b \neq \mathbf{0}$ (respectively, $f_{j} b \neq \mathbf{0}$ ), then we have $\operatorname{wt}\left(e_{j} b\right)=\operatorname{wt}(b)+\operatorname{cl}\left(\alpha_{j}\right)$ (respectively, $\left.\operatorname{wt}\left(f_{j} b\right)=\operatorname{wt}(b)-\operatorname{cl}\left(\alpha_{j}\right)\right)$. Therefore, from (5.2.3), we conclude that $\Phi_{i}: B_{\mathrm{cl}\left(\varpi_{i}\right)} \rightarrow \mathbb{B}\left(\varpi_{i}\right)_{\mathrm{cl}}$ is an isomorphism of $P_{\mathrm{cl}}$-crystals.

Let $\mathbf{i}=\left(i_{1}, i_{2}, \ldots, i_{n}\right)$ be an arbitrary sequence of elements of $I_{0}=\{1,2, \ldots, \ell-1\}$ such that $i_{1} \geqslant$ $i_{2} \geqslant \cdots \geqslant i_{n}$, and set $\lambda:=\sum_{k=1}^{n} \varpi_{i_{k}} \in P_{+}^{0}$; we denote this sequence $\mathbf{i}=\left(i_{1}, i_{2}, \ldots, i_{n}\right)$ by $\lambda^{+}$when we regard it as a partition (or Young diagram). In the following, we identify an element $\mu \in \operatorname{cl}\left(P_{+}^{0}\right)$ that is the weight of an element of the $P_{\mathrm{cl}}$-crystal $\mathbb{B}_{\mathbf{i}}$ with the partition $\left({ }^{(1)} \mu,{ }^{(2)} \mu, \ldots,{ }^{(\ell)} \mu\right) \in\left(\mathbb{Z}_{\geqslant 0}\right)^{\ell}$ of $\left|\lambda^{+}\right|:=i_{1}+i_{2}+\cdots+i_{n}$ such that $\mu=\sum_{i=1}^{\ell-1}\left({ }^{(i)} \mu-{ }^{(i+1)} \mu\right) \operatorname{cl}\left(\varpi_{i}\right)$ and $\sum_{i=1}^{\ell}{ }^{(i)} \mu=\left|\lambda^{+}\right|$; note that the elements $\operatorname{cl}\left(\varpi_{i}\right), 1 \leqslant i \leqslant \ell-1$, of $\mathfrak{h}^{*} / \mathbb{Q} \delta$ are linearly independent over $\mathbb{Q}$. Because $\mathbb{B}\left(\varpi_{i}\right)_{\mathrm{cl}}$ is isomorphic as a $P_{\mathrm{cl}}$-crystal to $B_{\mathrm{cl}\left(\varpi_{i}\right)}$ through the map $\Phi_{i}: B_{\mathrm{cl}\left(\varpi_{i}\right)} \stackrel{\sim}{\rightarrow} \mathbb{B}\left(\varpi_{i}\right)_{\mathrm{cl}}$ and, in addition, $\operatorname{res}_{I_{0}} \mathbb{B}\left(\varpi_{i}\right)_{\mathrm{cl}}$ is connected for every $i \in I_{0}=\{1,2, \ldots, \ell-1\}$, we deduce from [NY97, Corollary 4.3] along with (5.2.1) that, for every $\mu \in \operatorname{cl}\left(P_{+}^{0}\right)$ that is the weight of an element of $\mathbb{B}_{\mathbf{i}}$, the KostkaFoulkes polynomial $K_{\mu^{t}, \lambda^{+}}(q)$ (as defined in [Mac95, ch. III, $\left.\S 6\right]$ ) associated to the conjugate (or transpose) $\mu^{\mathrm{t}}$ of the partition $\mu$ and the partition $\lambda^{+}$is identical to the following $1 \mathrm{dsum:}$

$$
X\left(\mathbb{B}_{\mathbf{i}}, \mu ; q^{-1}\right)=\sum_{\substack{b \in \mathbb{B}_{\mathbf{i}} \\ e_{j} b=\mathbf{0}\left(j \in I_{0}\right) \\ \text { wt } b=\mu}} q^{-D_{\mathbf{i}}(b)} .
$$

By combining this fact and Corollary 5.1.1 (along with (5.2.1)), we obtain the following expression for Kostka-Foulkes polynomials in terms of LS paths.

Corollary 5.2.4. Assume that $\mathfrak{g}$ is of type $A_{\ell-1}^{(1)}$, and keep the notation above. Let $\mu \in \operatorname{cl}\left(P_{+}^{0}\right)=$ $\sum_{i \in I_{0}} \mathbb{Z}_{\geqslant 0} \operatorname{cl}\left(\varpi_{i}\right)$ be the weight of an element of the $P_{\mathrm{cl}}$-crystal $\mathbb{B}_{\mathbf{i}}$. Then, the following equation holds:

$$
K_{\mu^{t}, \lambda^{+}}(q)=\sum_{\substack{\eta \in \mathbb{B}(\lambda)_{\mathrm{cl}} \\ e_{j} \eta=\mathbf{0}\left(j \in I_{0}\right) \\ \eta(1)=\mu}} q^{-\operatorname{Deg}(\eta)}
$$




\section{LS PATHS OF LEVEL-ZERO SHAPE AND 1DSUMS}

\section{REFERENCES}

AK97

BFKL06

Cha01

FL06

FL07

FSS07

GL04

HKOTT02

HKOTY99

Her06

Her07

HN06

HK02

Kac90

KKMMnN92 S.-J. Kang, M. Kashiwara, K. C. Misra, T. Miwa, T. Nakashima and A. Nakayashiki, Affine crystals and vertex models, in Infinite analysis: Part A, Advanced Series in Mathematical Physics, vol. 16, eds A. Tsuchiya, T. Eguchi and M. Jimbo (World Scientific, River Edge, NJ, 1992), 449-484.

Kas94 M. Kashiwara, Crystal bases of modified quantized enveloping algebra, Duke Math. J. 73 (1994), 383-413.

Kas95 M. Kashiwara, On crystal bases, in Representations of groups, CMS Conference Proceedings, vol. 16, eds B. N. Allison and G. H. Cliff (American Mathematical Society, Providence, RI, 1995), 155-197.

Kas02 M. Kashiwara, On level-zero representations of quantized affine algebras, Duke Math. J. 112 (2002), 117-175.

Kas05 M. Kashiwara, Level zero fundamental representations over quantized affine algebras and Demazure modules, Publ. Res. Inst. Math. Sci. 41 (2005), 223-250.

Kir85 A. N. Kirillov, Combinatorial identities and completeness of states of the Heisenberg magnet, J. Soviet Math. 30 (1985), 2298-2310.

Kir87 A. N. Kirillov, Completeness of states of the generalized Heisenberg magnet, J. Soviet Math. 36 (1987), 115-128. 


\section{LS PATHS OF LEVEL-ZERO SHAPE AND 1DSUMS}

Kir89

KKR88

KNT02

KR90

KSS02

Lit94

Lit95

Mac95

NS03

$\mathrm{NS} 05$

NS06

NS08

Nak03

Nak04

NY97

Oka07

Sch05

Sch07

SSh06

SSt06

A. N. Kirillov, Identities for the Rogers dilogarithmic function connected with simple Lie algebras, J. Soviet Math. 47 (1989), 2450-2459.

S. V. Kerov, A. N. Kirillov and N. Yu. Reshetikhin, Combinatorics, the Bethe ansatz and representations of the symmetric group, J. Soviet Math. 41 (1988), 916-924.

A. Kuniba, T. Nakanishi and Z. Tsuboi, The canonical solutions of the Q-systems and the Kirillov-Reshetikhin conjecture, Comm. Math. Phys. 227 (2002), 155-190.

A. N. Kirillov and N. Yu. Reshetikhin, Representations of Yangians and multiplicities of the inclusion of the irreducible components of the tensor product of representations of simple Lie algebras, J. Soviet Math. 52 (1990), 3156-3164.

A. N. Kirillov, A. Schilling and M. Shimozono, A bijection between Littlewood-Richardson tableaux and rigged configurations, Selecta Math. (N.S.) 8 (2002), 67-135.

P. Littelmann, A Littlewood-Richardson rule for symmetrizable Kac-Moody algebras, Invent. Math. 116 (1994), 329-346.

P. Littelmann, Paths and root operators in representation theory, Ann. of Math. (2) 142 (1995), 499-525.

I. G. Macdonald, Symmetric functions and Hall polynomials, second edition, Oxford Mathematical Monographs, (Clarendon Press, Oxford, New York, 1995).

S. Naito and D. Sagaki, Path model for a level-zero extremal weight module over a quantum affine algebra, Int. Math. Res. Not. 2003 (2003), 1731-1754.

S. Naito and D. Sagaki, Crystal of Lakshmibai-Seshadri paths associated to an integral weight of level zero for an affine Lie algebra, Int. Math. Res. Not. 2005 (2005), 815-840.

S. Naito and D. Sagaki, Path model for a level-zero extremal weight module over a quantum affine algebra. II, Adv. Math. 200 (2006), 102-124.

S. Naito and D. Sagaki, Crystal structure on the set of Lakshmibai-Seshadri paths of an arbitrary level-zero shape, Proc. London Math. Soc. (3) 96 (2008), 582-622.

H. Nakajima, $t$-analogs of q-characters of Kirillov-Reshetikhin modules of quantum affine algebras, Represent. Theory 7 (2003), 259-274.

H. Nakajima, Extremal weight modules of quantum affine algebras, in Representation theory of algebraic groups and quantum groups, Advanced Studies in Pure Mathematics, vol. 40, eds T. Shoji et al. (Mathematical Society of Japan, Tokyo, 2004), 343-369.

A. Nakayashiki and Y. Yamada, Kostka polynomials and energy functions in solvable lattice models, Selecta Math. (N.S.) 3 (1997), 547-599.

M. Okado, $X=M$ conjecture, in Combinatorial aspect of integrable systems, MSJ Memoirs, vol. 17, eds A. Kuniba and M. Okado (Mathematical Society of Japan, Tokyo, 2007), 43-73.

A. Schilling, A bijection between type $D_{n}^{(1)}$ crystals and rigged configurations, J. Algebra 285 (2005), 292-334.

A. Schilling, $X=M$ theorem: fermionic formulas and rigged configurations under review, in Combinatorial aspect of integrable systems, MSJ Memoirs, vol. 17, eds A. Kuniba and M. Okado (Mathematical Soceity of Japan, Tokyo, 2007), 75-104.

A. Schilling and M. Shimozono, $X=M$ for symmetric powers, J. Algebra 295 (2006), $562-610$.

A. Schilling and P. Sternberg, Finite-dimensional crystals $B^{2, s}$ for quantum affine algebras of type $D_{n}^{(1)}$, J. Algebraic Combin. 23 (2006), 317-354.

Satoshi Naito naito@math.tsukuba.ac.jp

Institute of Mathematics, University of Tsukuba, Tsukuba, Ibaraki 305-8571, Japan

Daisuke Sagaki sagaki@math.tsukuba.ac.jp

Institute of Mathematics, University of Tsukuba, Tsukuba, Ibaraki 305-8571, Japan 